Hipersuperfícies regradas e de Weingarten em formas espaciais

\author{
Barbara Corominas Valério
}

TESE APRESENTADA

$\mathrm{AO}$

INSTITUTO DE MATEMÁTICA E ESTATÍSTICA

DA

UNIVERSIDADE DE SÃO PAULO

PARA

OBTENÇÃO DO GRAU DE DOUTOR

EM

MATEMÁTICA

Área de Concentração: Geometria Diferencial Orientador: Prof. Dr. Antonio Carlos Asperti

Durante a elaboração deste trabalho o autor recebeu apoio financeiro da FAPESP Processo 99/11398-6

-São Paulo, dezembro de 2004- 


\title{
Hipersuperfícies regradas e de Weingarten em formas espaciais
}

\author{
Este exemplar corresponde à redação final \\ da Tese devidamente corrigida e defendida \\ por Barbara Corominas Valério \\ e aprovada pela comissão julgadora.
}

São Paulo, dezembro de 2004.

Comissão Julgadora

- Prof. Dr. Antonio Carlos Asperti (presidente)- IME-USP

- Profa Dr $\stackrel{a}{a}$ Rosa Maria S. Barreiro Chaves - IME-USP

- Prof. Dr. Francesco Mercuri-IMECC-UNICAMP

- Prof. Dr. Renato Hyuda de Luna Pedrosa - IMECC-UNICAMP

- Prof. Dr. Ruy Tojeiro de Figueiredo Junior -UFSCar 
À minha mãe, Dolores, e ao meu companheiro Gilberto. 


\section{Agradecimentos}

Ao meu orientador, Prof. Antonio Carlos Asperti, pela dedicação e paciência com que me orientou. Seus conhecimentos e questionamentos me ajudaram a alcançar mais este objetivo. Só lamento a nossa incompatibilidade futebolística, infelizmente ele é Palmeirense.

Aos professores Fabiano Gustavo Braga Brito, Rosa Maria dos Santos Barreiro Chaves e Francesco Mercuri, muito obrigada pelas sugestões feitas durante todo o meu trabalho. Em especial, quero agradecer novamente à professora Rosa M. B. Chaves e à professora Cláudia Cueva Candido pelo constante apoio, incentivo e carinho.

Aos meus amigos do IME, principalmente os da sala $140-B$, com os quais tenho compartilhado os últimos anos, obrigada por todos os momentos de descontração e por todas as palavras de carinho. Em particular quero agradecer aos meus amigos Cecília, Samuel (quebrei a tradição de um ano), Major, Maite, Mário, Raul, Irene Onnis, Zé e Irene.

Aos meus pais, pelo constante apoio e, em particular, à minha mãe por todas as palavras de conforto. Aos meus irmãos Montserrat e Artur, aos meus cunhados Rogério e Fernanda, e aos meus sobrinhos Natália, Lucas, Paloma e Nicolle que sempre me incentivaram, cada um da sua forma.

Ao meu grande amigo, companheiro, confidente e terapeuta Gilberto, obrigada por tudo, principalmente por estar sempre ao meu lado. 


\section{Abstract}

In 1865/66 Beltrami [Be] and Dini [Di] classified, independently, the ruled Weingrten surfaces in $\mathbb{R}^{3}$. More recently, in 1984, Dajczer and Tenenblat [DT] extended this classification in the case of hypersurfaces in $\mathbb{R}^{n+1}, n \geq 3$. This work generalize the above/previous results to space form, that is, classify the ruled Weingarten hypersurfaces in $\mathbb{S}^{n+1}$ and $\mathbb{H}^{n+1}, n \geq 2$. In $\mathbb{H}^{n+1}, n \geq 3$, the results are partial yet.

\section{Resumo}

Em 1865/66 Beltrami [Be] e Dini [Di] classificaram, independentemente, as superfícies regradas e de Weingarten em $\mathbb{R}^{3}$. Mais recentemente, em 1984, Dajczer e Tenenblat [DT] estenderam esta classificação para o caso de hipersuperfícies em $\mathbb{R}^{n+1}$. Este trabalho generaliza os resultados anteriores para formas espaciais, isto é, classifica as superfícies regradas e de Weingarten em $\mathbb{S}^{n+1}$ e $\mathbb{H}^{n+1}, n \geq 2$. Em $\mathbb{H}^{n+1}, n \geq 3$, os resultados ainda são parciais. 


\section{Sumário}

Introdução $\quad$ iii

1 Conceitos básicos 1

1.1 Generalidades sobre o espaço de Lorentz . . . . . . . . . . . . . . . 1

1.1.1 Hipersuperfícies em $\mathbb{L}^{n+1} \ldots \ldots \ldots \ldots$

1.2 Generalidades sobre $\mathbb{Q}^{n}(c) \ldots \ldots \ldots \ldots$

1.3 Generalidades sobre o espaço de De Sitter . . . . . . . . . . . 6

1.3.1 Hipersuperfícies tipo espaço no espaço de De Sitter . . . . . . 6

1.3.2 Geodésicas do espaço de De Sitter . . . . . . . . . . . 8

1.4 O Triedro de Frenet para curvas em $\mathbb{Q}^{3}(c) \ldots \ldots \ldots$

1.5 Superfícies helicoidais em formas espaciais . . . . . . . . . . . . 12

1.6 Subvariedades regradas em formas espaciais . . . . . . . . . . 17

1.7 Superfícies regradas no espaço de De Sitter . . . . . . . . . . . . . . . 19

1.8 Hipersuperfícies de Weingarten . . . . . . . . . . . . . . 20

1.9 Redução de codimensão . . . . . . . . . . . . . . . . . . 22

2 Superfícies regradas $\quad 26$

2.1 Superfícies regradas em $\mathbb{Q}^{3}(c) \ldots \ldots \ldots \ldots$

2.2 Superfícies regradas tipo espaço em $\mathbb{S}_{1}^{3} \ldots \ldots \ldots$

2.3 Equação de Codazzi para superfícies em $\mathbb{S}^{3}$ e $\mathbb{H}^{3} \ldots \ldots$. . . . . . 34

2.4 Superfícies regradas com curvatura Gaussiana constante em $\mathbb{Q}^{3}(c)$ e $\mathbb{S}_{1}^{3} \quad 37$ 
2.4.1 Superfícies regradas desenvolvíveis em $\mathbb{Q}^{3}(c) \ldots \ldots$

2.4.2 Superfícies regradas tipo espaço desenvolvíveis em $\mathbb{S}_{1}^{3} \ldots \ldots 2$

3 Superfícies regradas helicoidais em formas espaciais $\quad \mathbf{4 3}$

3.1 Uma caracterização das hélices em formas espaciais . . . . . . . . . . 43

3.2 Uma caracterização das superfícies regradas helicoidais em formas espaciais ......................... 47

4 Superfícies regradas e de Weingarten em formas espaciais $\quad \mathbf{5 4}$

4.1 Classificação das superfícies regradas e de Weingarten em $\mathbb{S}^{3} \ldots$. . . 54

4.2 Classificação das superfícies regradas e de Weingarten em $\mathbb{H}^{3} \ldots$. . . 60

5 Hipersuperfícies regradas e de Weingarten em formas espaciais $\quad 67$

5.1 Parametrização de Gauss de uma hipersuperfície em uma forma espacial 67

5.2 Classificação das hipersuperfícies regradas e de Weingarten em $\mathbb{S}^{n+1}$. 73

5.3 Resultados parciais obtidos sobre a classificação das hipersuperfícies regradas e de Weingarten em $\mathbb{H}^{n+1} \ldots \ldots$. . . . . . . 88

$\begin{array}{ll}\text { Referências Bibliográficas } & 95\end{array}$ 


\section{Introdução}

Uma hipersuperfície $M^{n}$ em uma forma espacial real $\mathbb{Q}^{n+1}(c), n \geq 2$, é regrada quando é folheada por $(n-1)$-subvariedades totalmente geodésicas de $\mathbb{Q}^{n+1}(c) . M^{n}$ é de Weingarten quando existe uma relação funcional não trivial entre as suas curvaturas média e escalar.

Em 1865/66 Beltrami [Be] e Dini [Di] classificaram, independentemente, as superfícies regradas e de Weingarten em $\mathbb{R}^{3}$. Em 1994, Kühnel [K] reescreve os trabalhos de Beltrami e Dini, onde enuncia o seguinte teorema:

Teorema 0.1 Seja $X$ a parametrização de uma superfície regrada não desenvolvível em $\mathbb{R}^{3}$. São equivalentes:

(i) $X$ é a parametrização de uma superfície de Weingarten;

(ii) as funções $Q, J, F$ são constantes;

(iii) $2 H \sqrt{ \pm Q}=\sqrt[4]{-K}(J \mp F \sqrt{-K})$ para constantes $Q, J, F$ onde o sinal é o sinal definido por $Q$;

(iv) $X$ é a parametrização de uma superfície regrada helicoidal definida pelo movimento helicoidal ("screw motion") de uma reta.

No enunciado acima, $Q, J, F$ são funções de $\mathbb{R}$ em $\mathbb{R}$ e estão definidas em (2.3). Temos então que as superfícies regradas e de Weingarten em $\mathbb{R}^{3}$, são exatamente as superfícies regradas helicoidais, ou seja, as superfícies regradas invariantes por um movimento helicoidal, ver definição (1.15).

Seguem-se ainda, da demonstração do teorema anterior, outras características das superfícies regradas e de Weingarten em $\mathbb{R}^{3}$. Em primeiro lugar, gostaríamos de observar que as superfícies regradas são parametrizadas através da função exponencial, isto é,

$$
\begin{aligned}
X(s, t) & =\exp _{e_{0}(s)}\left(t e_{1}(s)\right) \\
& =e_{0}(s)+t e_{1}(s),
\end{aligned}
$$


onde $e_{0}, e_{1}$ são curvas em $\mathbb{R}^{3}$ tais que $\left|\dot{e}_{1}\right|^{2}=1$ e $\left\langle\dot{e}_{0}, \dot{e}_{1}\right\rangle=0$. Esta parametrização é muito importante para o estudo das superfícies regradas, pois ela permite a visualização das folhas que regram a superfície. As funções mencionadas no teorema anterior, surgem de forma natural quando determinamos uma expressão para as curvaturas Gaussiana e média da superfície, ver (2.4), e elas estão diretamente relacionadas com a parametrização da superfície. Usando-se o fato das funções $Q, J, F$ serem constantes, segue-se que as superfícies regradas e de Weingarten possuem a característica que suas curvaturas Gaussiana e média são constantes em relação ao parâmetro translacional, veja definição (1.19). Além disso, observou-se o fato que as superfícies regradas helicoidais em $\mathbb{R}^{3}$ possuem curvatura Gaussiana e média constantes em relação a um dos parâmetros usados para a parametrização da superfície.

O primeiro objetivo deste trabalho foi generalizar o resultado obtido por Beltrami e Dini para superfícies regradas em formas espaciais, $\mathbb{Q}^{3}(c), c \neq 0$. Sem perda de generalidade, supomos que $\mathbb{Q}^{3}(c)$ é o espaço euclidiano $\mathbb{R}^{3}$, se $c=0$; é a esfera unitária $\mathbb{S}^{3}=\left\{x=\left(x_{1}, x_{2}, x_{3}, x_{4}\right) \in \mathbb{R}^{4}: \sum_{j=1}^{4} x_{j}^{2}=1\right\}$, se $c=1$; ou é. o espaço hiperbólico $\mathbb{H}^{3}=\left\{x=\left(x_{1}, x_{2}, x_{3}, x_{4}\right) \in \mathbb{R}^{4}:-x_{1}^{2}+\sum_{j=2}^{4} x_{j}^{2}=-1, x_{1}>1\right\}$, se $c=-1$. Observando o comportamento das superfícies regradas e de Weingarten em $\mathbb{R}^{3}$, várias perguntas foram feitas a respeito de qual seria o comportamento das superfícies regradas e de Weingarten em $\mathbb{S}^{3}$ e $\mathbb{H}^{3}$, as mais importantes foram:

1. Quais os exemplos mais simples de superfícies regradas e de Weingarten em $\mathbb{Q}^{3}(c) ?$

2. É condição suficiente, para que uma superfície regrada em $\mathbb{S}^{3}$ e $\mathbb{H}^{3}$ seja helicoidal, que ela possua curvatura Gaussiana e média constantes em relação a um dos parâmetros?

3. Também é condição necessária que uma superfície regrada em $\mathbb{S}^{3}$ e $\mathbb{H}^{3}$ possua curvatura Gaussiana e média constantes em relação a um dos parâmetros para que seja de Weingarten?

A primeira pergunta é facilmente resolvida quando se observa que as curvaturas Gaussiana e média de uma superfície regrada satisfazem uma relação não trivial se, e somente se, satisfazem à igualdade $K_{s} H_{t}-K_{t} H_{s}=0$, onde $s, t$ são parâmetros da superfície, ver Proposição (1.21). Segue-se então que os exemplos mais simples de superfícies regradas e de Weingarten, são as superfícies com curvatura Gaussiana ou média constantes, e as superfícies com curvatura Gaussiana e média constantes em relação a um mesmo parâmetro. Convém mencionar que as subvariedades regradas com curvatura média constante em formas espaciais são completamente classificadas nos trabalhos [BDJ] e [BD]. 
Se a resposta para a segunda questão for afirmativa, temos como no caso euclidiano, que as superfícies regradas helicoidais em $\mathbb{Q}^{3}(c), c \neq 0$, são exemplos de superfícies regradas e de Weingarten em $\mathbb{Q}^{3}(c)$, pois pela primeira questão, é condição suficiente para que uma superfície regrada seja de Weingarten, que possua curvatura Gaussiana e média constantes em relação a um dos parâmetros. Se a resposta para a terceira questão for afirmativa, teremos que as superfícies regradas helicoidais são todos os exemplos de superfícies regradas e de Weingarten em $\mathbb{Q}^{3}(c), c \neq 0$, pois a condição sobre as curvaturas Gaussiana e média passa a ser necessária. Observamos que a segunda e a terceira questão são respondidas de forma afirmativa.

No Capítulo 1 falamos dos conceitos básicos sobre a teoria usada para o desenvolvimento do trabalho.

No Capítulo 2, mais precisamente nas seções 2.1 e 2.3, estudamos as superfícies regradas em formas espaciais, com o objetivo de fornecer as relações geométricas que elas satisfazem. Observamos que as superfícies regradas no caso esférico e hiperbólico, também são parametrizadas através da função exponencial, ver (2.1). Além disso, na seção 2.4 estudamos as superfícies regradas com curvatura Gaussiana constante e estendemos a definição de superfície desenvolvível. Ainda neste capítulo, estudamos algumas propriedades da superfícies regradas no espaço de De Sitter $\mathbb{S}_{1}^{3}$, que são usadas no capítulo 5 .

No Capítulo 3 estudamos as superfícies regradas helicoidais em formas espaciais e mostramos que é suficiente, para que uma superfície regrada seja helicoidal, que possua curvatura Gaussiana e média constante em relação ao parâmetro translacional. Para mostrar tal caracteristica foi fundamental o estudo das hélices em formas espaciais.

No Capítulo 4 estudamos as superfícies regradas e de Weingarten em formas espaciais, e os teoremas encontrados (4.2) e (4.4) são a extensão natural do resultado obtido por Beltrami e Dini para $\mathbb{S}^{3}$ e $\mathbb{H}^{3}$, respectivamente. Observamos, no entanto, que foi preciso adicionar uma hipótese técnica nos teoremas citados.

Feita a classificação das superfícies regradas e de Weingarten em formas espaciais, o passo seguinte foi estudar o caso das hipersuperfícies, $M^{n}$ em $\mathbb{Q}^{n+1}(c), n \geq 3$.

Em 1989 Dajczer e Tenenblat [DT] classificaram as hipersuperfícies regradas e de Weingarten em $\mathbb{R}^{n+1}$. Para obter tal classificação, eles usaram como ferramenta a parametrização de Gauss para hipersuperfícies com nulidade relativa constante, descrita por Dajczer e Gromoll em [DG]. As hipersuperfícies com índice de nulidade relativa constante estão relacionadas com as hipersuperfícies regradas, pelo simples resultado de que em um ponto de uma hipersuperfície regrada, a curvatura escalar é diferente da curvatura do ambiente se, e somente se, neste ponto o índice de nulidade 
relativa é igual a $n-2$, ver observações 5.4 e 5.5 .

No Capítulo 5, classificamos as hipersuperfícies regradas e de Weingarten em $\mathbb{Q}^{n+1}(c), c \neq 0$, mais especificamente, na seção 5.1 falamos sobre a parametrização de Gauss descrita por Dajczer e Gromoll, na seção 5.2, mostramos que o resultado obtido por Dajczer e Tenenblat se estende naturalmente para o caso esférico, isto é, para o caso de hipersuperfície em $\mathbb{S}^{n+1}$. Na seção 5.3 , estendemos o resultado para o caso de hipersuperfícies em $\mathbb{H}^{n+1}$, no entanto, os resultados obtidos são parciais, isto é, a classificação ainda não está completa. As dificuldades encontradas na seção 5.3, devem-se ao fato que quando tomamos a aplicação de Gauss da hipersuperfície em $\mathbb{H}^{n+1}$, a sua imagem está contida no espaço de De Sitter, $\mathbb{S}_{1}^{n+1}$, que é uma hipersuperfície tipo tempo do espaço de Lorentz $(n+2)$-dimensional.

Por fim, gostariamos de observar que alguns cálculos feitos nos capítulos 3 e 4 foram realizados com o auxílio do software Mathematica. 


\section{Capítulo 1}

\section{Conceitos básicos}

O objetivo deste primeiro capítulo é fixar a notação e apresentar alguns conceitos que serão usados ao longo deste trabalho.

\subsection{Generalidades sobre o espaço de Lorentz}

Seja $\mathbb{L}^{n+1}$ o espaço de Lorentz-Minkowski $n+1$-dimensional, isto é, o espaço euclideano $\mathbb{R}^{n+1}=\left\{\left(x_{1}, \ldots x_{n+1}\right) / x_{i} \in \mathbb{R}\right\}$ munido com a métrica pseudo-Riemanniana $g_{-1}(x, y)=-x_{1} y_{1}+\sum_{i=2}^{n+1} x_{i} y_{i}$. Observamos que $g_{-1}$ é uma forma bilinear simétrica, não degenerada de índice 1 sobre $\mathbb{R}^{n+1}$, onde o índice $\nu$ de uma forma bilinear simétrica $\mathcal{B}$ sobre um $\mathbb{R}$-espaço vetorial $V$, é a maior dimensão de qualquer subespaço $W \subset V$ no qual se $w \in W, w \neq 0$ então $\mathcal{B}(w, w)<0$, isto é, $\mathcal{B}$ é negativa definida em $W$. $\mathbb{L}^{n+1}$ :

Segue-se do fato da métrica $g_{-1}$ ter índice 1 que existem três tipos de vetores em

Definição 1.1 Seja $v \in \mathbb{L}^{n+1}$. Dizemos que vé tipo

$$
\begin{aligned}
& \text { espaço, se } g_{-1}(v, v)>0 \text { ou } v=0 \text {, } \\
& \text { tempo, se } g_{-1}(v, v)<0 \text {, } \\
& \text { luz, se } g_{-1}(v, v)=0 \text { e } \quad v \neq 0 \text {. }
\end{aligned}
$$


Baseado no tipo causal de um vetor em $\mathbb{L}^{n+1}$, existe uma generalização natural para o tipo causal de um subespaço vetorial de $\mathbb{I}^{n+1}$. Observe que o tipo causal de um vetor coincide com o tipo causal do subespaço por ele gerado. Seja então $W$ um subespaço vetorial de $\mathbb{L}^{n+1}$. Existem três possibilidades mutuamente exclusivas para $W:$

$$
\begin{gathered}
g_{-\left.1\right|_{W}} \text { é positiva-definida; } W \text { é dito tipo espaço. } \\
g_{-\left.1\right|_{W}} \text { é não-degenerada de índice } 1 ; W \text { é dito tipo tempo. } \\
g_{-\left.1\right|_{W}} \text { é degenerada; } W \text { é dito tipo luz. }
\end{gathered}
$$

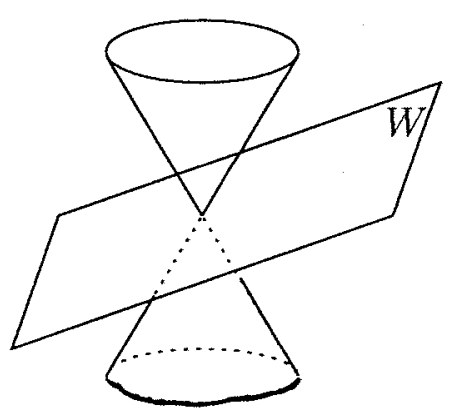

1

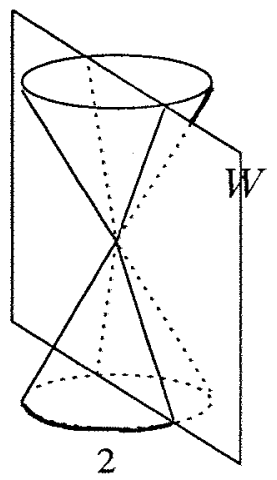

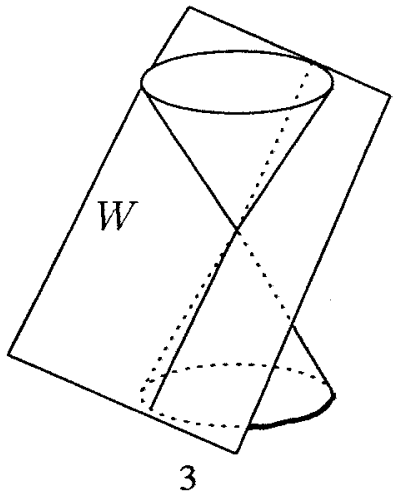

Figura 1.1: $W$ é um subespaço tipo espaço em 1, tipo tempo em 2 e tipo luz em 3.

A seguir enunciaremos, sem demonstrar, alguns resultados sobre $\mathbb{L}^{n+1}$, ver $[0]$.

Proposição 1.2 Se $W$ é um k-subespaço de $\mathbb{L}^{n+1}$, então $n+1=\operatorname{dim} W+\operatorname{dim} W^{\perp}$ $e W^{\perp}=W$, onde $W^{\perp}=\left\{v \in \mathbb{L}^{n+1} / g_{-1}(v, w)=0, \forall w \in W\right\}$.

Proposição 1.3 Se v é um vetor tipo tempo no espaço de Lorentz, então o subespaço $v^{\perp}$ é tipo espaço e $\mathbb{L}^{n+1}=\mathbb{R} v+v^{\perp}$. 
Proposição 1.4 Se $W$ é um subespaço de $\mathbb{L}^{n+1}$, então $g_{-1 \mid}$ é não-degenerada de indice 1 se, e somente se, $\left.g_{-1}\right|_{W \perp}$ é positiva-definida.

Proposição 1.5 Seja $W$ um $k$-subespaço de $\mathbb{L}^{n+1}$, com $k \geq 2$. São equivalentes:

i) $g_{-\left.1\right|_{W}}$ é não degenerada de índice 1 .

ii) $W$ contém dois vetores nulos, linearmente independentes.

iii) $W$ contém um vetor tipo tempo.

Por fim lembramos que analogamente ao caso Riemanniano, é possível definir em $\mathbb{L}^{n+1}$ uma única conexão $\bar{\nabla}$ denominada conexão de Levi-Civita de $\mathbb{L}^{n+1}$, melhor dizendo:

Teorema 1.6 Existe uma única aplicação $\bar{\nabla}: \chi\left(\mathbb{L}^{n+1}\right) \times \chi\left(\mathbb{L}^{n+1}\right) \rightarrow \chi\left(\mathbb{L}^{n+1}\right)$ tal que

1. $\bar{\nabla}_{V} W$ é $\mathcal{F}\left(\mathbb{L}^{n+1}\right)$-linear em $V$.

2. $\bar{\nabla}_{V} W$ é $\mathbb{R}$-linear em $W$.

3. $\bar{\nabla}_{V}(f W)=V[f] W+f \bar{\nabla}_{V} W$, para $f \in \mathcal{F}\left(\mathbb{L}^{n+1}\right)$.

4. $[V, W]=\bar{\nabla}_{V} W-\bar{\nabla}_{W} V$.

5. $X g_{-1}(V, W)=g_{-1}\left(\bar{\nabla}_{X} V, W\right)+g_{-1}\left(V, \bar{\nabla}_{X} W\right), \forall X, V, W \in \chi\left(\mathbb{L}^{n+1}\right)$.

$\bar{\nabla} e ́$ denominada conexão de Levi-Civita de $\mathbb{L}^{n+1}$.

\subsubsection{Hipersuperfícies em $\mathbb{L}^{n+1}$}

Definição 1.7 Dizemos que $M$ é uma hipersuperfície em $\mathbb{L}^{n+1}$ de tipo espaço, se a métrica induzida tem indice zero, isto é, se $g_{-\left.1\right|_{M}}$ é Riemanniana, e dizemos que é de tipo tempo, se a métrica induzida tem índice 1 , isto é, se $g_{-\left.1\right|_{M}}$ é Lorentziana.

Neste trabalho aparecerão duas hipersuperfícies de $\mathbb{L}^{n+1}$ conhecidas como o espaço hiperbólico, e o espaço de De Sitter.

- Espaço hiperbólico 


$$
\mathbb{H}^{n}(c)=\left\{x=\left(x_{1}, x_{2}, \ldots x_{n+1}\right) \in \mathbb{R}^{n+1} / g_{-1}(x, x)=-c^{2}, x_{1}>0\right\}
$$

é uma hipersuperfície tipo espaço de $\mathbb{L}^{n+1}$, denominada espaço hiperbólico de dimensão $n$.

- Espaço de De Sitter

$$
\mathbb{S}_{1}^{n}(c)=\left\{x=\left(x_{1}, x_{2}, \ldots x_{n+1}\right) \in \mathbb{R}^{n+1} / g_{-1}(x, x)=c^{2}\right\}
$$

é uma hipersuperfície tipo tempo de $\mathbb{I}^{n+1}$, denominada espaço de De Sitter de dimensão $n$ ou esfera $n$ dimensional de $\mathbb{L}^{n+1}$ de raio $c$.

Sabe-se que $\mathbb{H}^{n}(c)$ e $\mathbb{S}_{1}^{n}(c)$ são variedades completas, simplesmente conexas (exceto $\mathbb{S}_{1}^{2}(c)$ ), com curvatura seccional constante $-\frac{1}{c^{2}}$ e $\frac{1}{c^{2}}$, respectivamente. Sabe-se ainda, que $\mathbb{H}^{n}(c)$ juntamente com o espaço euclideano $\mathbb{R}^{n}$, e a esfera euclidiana $\mathbb{S}^{n}$, são as únicas variedades Riemannianas completas, simplesmente conexas com curvatura seccional constante. Baseados neste comportamento, estudaremos hipersuperfícies em $\mathbb{Q}^{n}(c)$ (definido a seguir) e hipersuperfícies em $\mathbb{S}_{1}^{n}(c)$.

\subsection{Generalidades sobre $\mathbb{Q}^{n}(c)$}

Usaremos a notação $\mathbb{Q}^{n}(c)$ para indicar a variedade Riemanniana $n$ dimensional, completa, simplesmente conexa com curvatura seccional constante $c=0, \pm 1$. Podemos assumir, sem perda de generalidade, que $\mathbb{Q}^{n}(c)$ é o espaço euclideano $\mathbb{R}^{n}$, se $c=0$; é a esfera unitária $\mathbb{S}^{n}=\left\{x=\left(x_{1}, x_{2}, \ldots x_{n+1}\right) \in \mathbb{R}^{n+1}: \sum_{j=1}^{n+1} x_{j}^{2}=1\right\}$, se $c=1$; ou é o espaço hiperbólico $\mathbb{H}^{n}=\left\{x=\left(x_{1}, x_{2}, \ldots x_{n+1}\right) \in \mathbb{R}^{n+1}:-x_{1}^{2}+\sum_{j=2}^{n+1} x_{j}^{2}=\right.$ $\left.-1, x_{1}>1\right\}$, se $c=-1$. Será conveniente, em alguns casos, identificarmos o espaço $\mathbb{R}^{n}$ com o hiperplano $\left\{\left(x_{1}, x_{2}, \ldots x_{n}, 1\right)\right\}$ de $\mathbb{R}^{n+1}$.

Seja $f: M^{m} \rightarrow \mathbb{Q}^{n}(c), 2 \leq m<n$, uma imersão isométrica de uma variedade diferenciável em $\mathbb{Q}^{n}(c)$. Como localmente $f$ é um mergulho, podemos considerar o espaço tangente a $M^{m}$ em $p$ como um subespaço do espaço tangente a $\mathbb{Q}^{n}(c)$ em $f(p)=p$, ou seja, $T_{p} \mathbb{Q}^{n}(c)=T_{p} M \oplus T_{p} M^{\perp}$, onde $T_{p} M^{\perp}$ é o complemento ortogonal de $T_{p} M$ em $T_{p} \mathbb{Q}^{n}(c)$. Desta decomposição obtemos o fibrado normal a $M$, denotado por $T M^{\perp}$. Seja ainda $T M$ o fibrado tangente a $M$. 
Denotando-se por $\nabla$ e $\bar{\nabla}$ as conexões de Levi-Civita de $M$ e $\mathbb{Q}^{n}(c)$, respectivamente, obtemos da decomposição anterior, as fórmulas de Gauss e Weingarten dadas respectivamente, por:

$$
\begin{gathered}
\bar{\nabla}_{X} Y=\nabla_{X} Y+\alpha(X, Y), \\
\bar{\nabla}_{X} \xi=-A_{\xi} X+\nabla_{X}^{\perp} \xi,
\end{gathered}
$$

onde $X, Y \in \chi(M), \xi \in \chi(M)^{\perp}, \alpha: T_{p} M \times T_{p} M \rightarrow T_{p} M^{\perp}$ é a segunda forma fundamental da imersão $f$ em $p \in M, A_{\xi}$ é o operador forma relativo à segunda forma fundamental de $f$ ao longo de $\xi$ e $\nabla \frac{1}{X} \xi=\left(\bar{\nabla}_{X} \xi\right)^{\perp}$ é a conexão normal de $f$.

Denotemos por $R(\bar{R})$ o tensor de curvatura de $M\left(\mathbb{Q}^{n}(c)\right)$ e por $R^{\perp}$ o tensor de curvatura normal de $T M^{\perp}$, definidos por

$$
R(X, Y) Z=\nabla_{X} \nabla_{Y} Z-\nabla_{Y} \nabla_{X} Z-\nabla_{[X, Y]} Z
$$

$\mathrm{e}$

$$
R^{\perp}(X, Y) \xi=\nabla_{X}^{\perp} \nabla_{Y}^{\perp} \xi-\nabla_{Y}^{\perp} \nabla_{X}^{\perp} \xi-\nabla_{[X, Y]}^{\perp} \xi
$$

onde $X, Y, Z \in \chi(M)$ e $\xi \in \chi(M)^{\perp}$.

Das duas fórmulas anteriores, derivam-se as equações básicas que uma imersão isométrica deve verificar. Como estamos considerando imersões isométricas em $\mathbb{Q}^{n}(c)$, isto é, em um espaço com curvatura seccional constante, as equações que se obtém são:

Equação de Gauss:

$$
\begin{aligned}
\langle R(X, Y) Z, W\rangle & =c\langle(\langle Y, Z\rangle X-\langle X, Z\rangle Y) Z, W\rangle+ \\
& +\langle\alpha(X, W), \alpha(Y, Z)\rangle-\langle\alpha(X, Z), \alpha(Y, W)\rangle .
\end{aligned}
$$

Equação de Codazzi:

$$
\left(\nabla_{X}^{\perp} \alpha\right)(Y, Z)=\left(\nabla_{Y}^{\perp} \alpha\right)(X, Z)
$$

ou equivalentemente

$$
\left(\nabla_{X} A\right)(Y, \xi)=\left(\nabla_{Y} A\right)(X, \xi)
$$

Equação de Ricci:

$$
R^{\perp}(X, Y) \xi=\alpha\left(X, A_{\xi} Y\right)-\alpha\left(A_{\xi} X, Y\right),
$$

ou equivalentemente

$$
\left\langle R^{\perp}(X, Y) \xi, \eta\right\rangle=\left\langle\left[A_{\xi}, A_{\eta}\right] X, Y\right\rangle .
$$


onde

$$
\left(\nabla_{X}^{1} \alpha\right)(Y, Z)=\nabla_{X}^{\perp} \alpha(Y, Z)-\alpha\left(\nabla_{X} Y, Z\right)-\alpha\left(Y, \nabla_{X} Z\right)
$$

$\mathrm{e}$

$$
\left(\nabla_{X} A\right)(Y, \xi)=\nabla_{X} A_{\xi} Y-A_{\xi} \nabla_{X} Y-A_{\nabla_{X}^{\frac{1}{X}} \xi} Y
$$

Observamos que estamos usando $\langle$,$\rangle para representar a métrica induzida de \mathbb{Q}^{n}(c)$ em $M^{m}$, nos três casos.

Denotando-se, respectivamente, por $K(X, Y)=\langle R(X, Y) X, Y\rangle$ e $\bar{K}(X, Y)=$ $\langle\bar{R}(X, Y) X, Y\rangle$ a curvatura seccional de $M$ e $\mathbb{Q}^{n}(c)$, em relação ao plano gerado pelos campos ortonormais $X$ e $Y$, segue-se pela equação de Gauss que

$$
K(X, Y)=\bar{K}(X, Y)+\langle\alpha(X, X), \alpha(Y, Y)\rangle-|\alpha(X, Y)|^{2} .
$$

\subsection{Generalidades sobre o espaço de De Sitter}

\subsubsection{Hipersuperfícies tipo espaço no espaço de De Sitter}

O espaço de De Sitter admite hipersuperfícies de tipo espaço, tipo tempo e tipo luz, no entanto discutiremos apenas o primeiro caso, pois será com este tipo de hipersuperfície que trabalharemos na seção 5.3. Sem perda de generalidade, consideraremos $\mathbb{S}_{1}^{n+1}(1) \cong \mathbb{S}_{1}^{n+1}$ e também usaremos $<,>$ para representar a métrica lorentziana induzida.

Seja $f: M^{n} \rightarrow \mathbb{S}_{1}^{n+1} \subset \mathbb{L}^{n+2}$ uma imersão isométrica tipo espaço, e denotemos por $\nabla, \bar{\nabla}$ e $\tilde{\nabla}$ as conexões de Levi-Civita de $M^{n}, \mathbb{S}_{1}^{n+1}$ e $\mathbb{L}^{n+2}$, respectivamente. Denotemos ainda por $N$ o campo normal unitário de $M^{n}$ em $\mathbb{S}_{1}^{n+1}$ e por $f$ o campo unitário (vetor posição) de $\mathbb{S}_{1}^{n+1}$ em $\mathbb{L}^{n+2}$, donde segue-se que $\langle f, f\rangle=1$ e $\langle N, N\rangle=$ -1 .

Para todo ponto $p \in M^{n}$, e identificando $f(p)=p$, podemos analogamente ao caso Riemanniano, fazer a seguinte identificação

$$
T_{p} \mathbb{S}_{1}^{n+1}=T_{p} M^{n} \oplus \operatorname{span}\{N(p)\} .
$$

Temos então a fórmula de Gauss $\bar{\nabla}_{X} Y=\nabla_{X} Y+\alpha(X, Y)$, onde $X, Y \in \chi(M)$ e $\alpha$ denota a segunda forma fundamental da imersão $f$. A fórmula de Gauss pode ainda 
ser reescrita como

$$
\bar{\nabla}_{X} Y=\nabla_{X} Y-\langle A X, Y\rangle N
$$

onde $A$ é o operador de Weingarten da imersão $f$ relativo ao campo $N$. A fórmula de Weingarten é dada pela relação $\bar{\nabla}_{X} \xi=\left(\bar{\nabla}_{X} \xi\right)^{T}+\left(\bar{\nabla}_{X} \xi\right)^{\perp}$, onde $\xi$ é um campo normal a imersão $f$. Como $\xi=N$ segue-se que a fórmula de Weingarten se reescreve como

$$
\bar{\nabla}_{X} N=\left(\bar{\nabla}_{X} N\right)^{T}=-A X
$$

Denotando por $R(\bar{R})$ o tensor de curvatura de $M\left(\mathbb{S}_{1}^{n+1}\right)$, temos que a equação de Gauss de $M$ é dada por

$$
\begin{gathered}
\langle R(V, W) X, Y\rangle=\langle\bar{R}(V, W) X, Y\rangle+\langle\alpha(V, X), \alpha(W, Y)\rangle+ \\
-\langle\alpha(V, Y), \alpha(W, X)\rangle .
\end{gathered}
$$

Segue-se ainda, denotando-se respectivamente por $K(X, Y)=\langle R(X, Y) X, Y\rangle$ e $\bar{K}(X, Y)=\langle\bar{R}(X, Y) X, Y\rangle$ as curvaturas seccionais de $M$ e $\mathbb{S}_{1}^{n+1}$, que

$$
K(X, Y)=\bar{K}(X, Y)+\langle\alpha(X, X), \alpha(Y, Y)\rangle-\langle\alpha(X, Y), \alpha(X, Y)\rangle
$$

onde $X$ e $Y$ são campos diferenciáveis sobre $M$ que geram um plano não degenerado. Observe que no caso que iremos estudar, não existem subespaços degenerados do espaço tangente.

Denotando por $\nabla^{\perp}: \chi(M) \times \chi(M)^{\perp} \rightarrow \chi(M)^{\perp}$ a conexão normal de $M$ definida por $\nabla_{X}^{\perp} \xi=\left(\bar{\nabla}_{X} \xi\right)^{\perp}$ onde $X \in \chi(M)$ e $\xi \in \chi(M)^{\perp}$, temos que a equação de Codazzi de $M$ é dada por

$$
\left(\nabla_{V}^{\perp} \alpha\right)(W, X)=\left(\nabla_{W}^{\perp} \alpha\right)(V, X), \quad V, W, X \in \chi(M)
$$

onde

$$
\left(\nabla_{V}^{\perp} \alpha\right)(W, X)=\nabla_{V}^{\perp}(\alpha(W, X))-\alpha\left(\nabla_{V} W, X\right)-\alpha\left(W, \nabla_{V} X\right)
$$

ou equivalentemente

$$
\left(\nabla_{V} A\right)(W, \xi)=\left(\nabla_{W} A\right)(V, \xi)
$$

onde

$$
\left(\nabla_{V} A\right)(W, \xi)=\nabla_{V} A_{\xi} W-A_{\xi} \nabla_{V} W-A_{\nabla_{V} \frac{1}{\xi}} W
$$




\subsubsection{Geodésicas do espaço de De Sitter}

Definição 1.8 Uma geodésica em uma variedade semi-Riemanniana, $M$, é uma curva $\gamma: I \rightarrow M$ cujo campo vetorial $\dot{\gamma}(s)$ é paralelo, isto é, $\frac{D \dot{\gamma}}{d s}=0$.

Definição 1.9 Uma curva $\gamma: I \rightarrow M$ em uma variedade semi-Riemanniana, $M$, é dita de tipo espaço se o vetor velocidade $\dot{\gamma}(s)$ é tipo espaço para todo $s \in I$. De forma análoga, podemos definir uma curva tipo tempo e tipo luz.

Uma curva qualquer em uma variedade semi-Riemanniana, não possui necessariamente um tipo causal, isto é, seu vetor velocidade não possui necessariamente o mesmo tipo causal em todo ponto. As geodésicas, no entanto, possuem um tipo causal, pois o vetor tangente à curva é paralelo, e o transporte paralelo de um vetor preserva o seu tipo causal.

Dependendo do tipo causal de uma geodésica, temos o seguinte resultado:

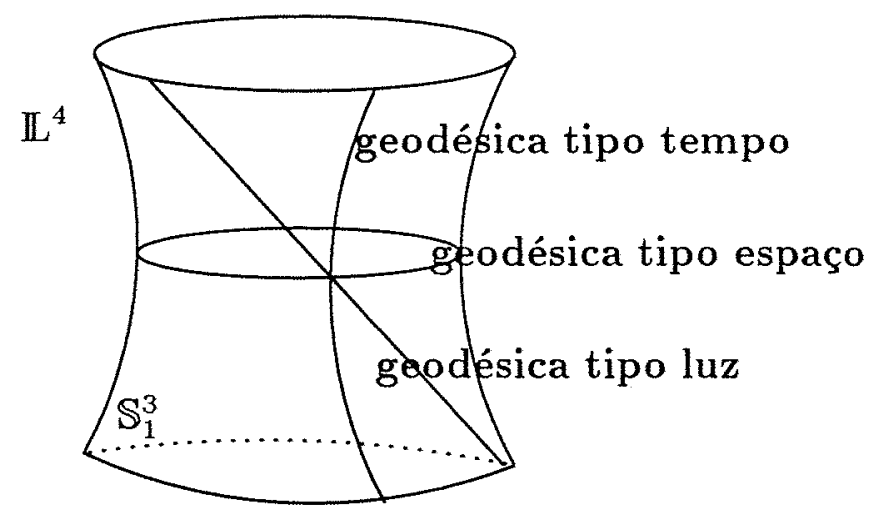

Figura 1.2: Geodésicas no espaço de De Sitter.

Proposição 1.10 Seja $\gamma$ a parametrização de uma geodésica em $\mathbb{S}_{1}^{n+1}$. Então podemos ter:

i) Se $\gamma$ é tipo tempo, então $\gamma$ é a parametrização de um ramo de uma hipérbole em $\mathbb{I L}^{n+2}$.

ii) Se $\gamma$ é tipo luz, então $\gamma$ é a parametrização de uma reta, isto é, é uma geodésica $e m \mathbb{L}^{n+2}$.

iii) Se $\gamma$ é tipo espaço, então $\gamma$ é a parametrização de uma elipse em $\mathbb{L}^{n+2}$. 
Demonstração:Ver [O].

\section{$1.4 \mathrm{O}$ Triedro de Frenet para curvas em $\mathbb{Q}^{3}(c)$}

Denotemos genericamente por $\mathbb{F}^{4}$ os espaços $\mathbb{R}^{4} \mathrm{e} \mathbb{L}^{4}$. Dados três vetores $u_{1}, u_{2}, u_{3}$ pertencentes a $\mathbb{F}^{4}$ existe um único vetor $u_{1} \wedge u_{2} \wedge u_{3}$ em $\mathbb{F}^{4}$ tal que para todo $z \in \mathbb{F}^{4}$

$$
\left\langle z, u_{1} \wedge u_{2} \wedge u_{3}\right\rangle:=\operatorname{det}\left(z, u_{1}, u_{2}, u_{3}\right)
$$

onde $\langle$,$\rangle denota a métrica em \mathbb{F}^{4}$. O vetor $u_{1} \wedge u_{2} \wedge u_{3}$ é chamado produto vetorial de $u_{1}, u_{2}$ e $u_{3}$ e é obtido da seguinte forma:

$$
u_{1} \wedge u_{2} \wedge u_{3}:=\left|\begin{array}{llll}
c e_{1} & e_{2} & e_{3} & e_{4} \\
u_{11} & u_{12} & u_{13} & u_{14} \\
u_{21} & u_{22} & u_{23} & u_{24} \\
u_{31} & u_{32} & u_{33} & u_{34}
\end{array}\right|
$$

onde $\left\{e_{1}, e_{2}, e_{3}, e_{4}\right\}$ denota a base canônica de $\mathbb{F}^{4}, u_{i}=\left(u_{i 1}, u_{i 2}, u_{i 3}, u_{i 4}\right)$ e $c=-1$, se $\mathbb{F}^{4}=\mathbb{L}^{4}$ e $c=1$, se $\mathbb{F}^{4}=\mathbb{R}^{4}$.

Com a definição anterior, verifica-se que o produto vetorial $u_{1} \wedge u_{2} \wedge u_{3}$ satisfaz as seguintes propriedades:

1. $u_{1} \wedge u_{2} \wedge u_{3}$ é simultaneamente ortogonal aos três vetores $u_{1}, u_{2}, u_{3}$;

2. $\left|u_{1} \wedge u_{2} \wedge u_{3}\right|^{2}=c \operatorname{det}\left(\left\langle u_{i}, u_{j}\right\rangle\right)$;

3. é antisimétrico, isto é, a troca de posição entre seus elementos implica na mudança de orientação do vetor;

4. o produto vetorial entre três vetores é nulo se, e somente se, pelo menos dois dos vetores são linearmente dependentes;

5. é bilinear em cada fator.

Ao estudarmos uma curva é fundamental conhecermos o comportamento de suas funções curvatura. Quando estamos em dimensão três, estas funções são as funções 
curvatura e torção. Determinemos o triedro de Frenet associado a uma curva em $\mathbb{Q}^{3}(c)$. Como o caso $c=0$ é bem conhecido, faremos apenas os casos $c= \pm 1$.

Seja $\gamma=\gamma(s): I \subset \mathbb{R} \longrightarrow \mathbb{Q}^{3}(c)$ uma curva regular em $\mathbb{Q}^{3}(c)$ com velocidade unitária. Denotando $\mathrm{T}(s)=\dot{\gamma}(s)$, definimos a curvatura de $\gamma$ por

$$
\kappa(s):=\left|\bar{\nabla}_{\mathrm{T}(s)} \mathrm{T}(s)\right|,
$$

onde $\bar{\nabla}$ é a conexão de Levi-Civita de $\mathbb{Q}^{3}(c)$. Se $\kappa(s) \neq 0$, definimos

$$
\mathrm{N}(s):=\frac{\bar{\nabla}_{\mathrm{T}(s)} \mathrm{T}(s)}{\kappa(s)}, \quad \mathrm{B}(s):=\mathrm{T}(s) \wedge \mathrm{N}(s) \wedge \gamma(s),
$$

onde em $B(s)$ é usado o produto vetorial definido acima. É imediato verificar que $\{T, N, B\}$ define um referencial ortonormal, o qual é chamado triedro de Frenet associado a curva $\gamma$.

Denotando por '.' a derivada em relação a variável $s$ em $\mathbb{F}^{4}$, e por $\bar{D}$ a derivada covariante em $\mathbb{Q}^{3}(c)$, segue-se no caso $c=1$ que

$$
\frac{\bar{D} \mathrm{~B}}{d s}=\dot{\mathrm{B}}-\langle\dot{\mathrm{B}}, \gamma\rangle \gamma
$$

onde

$$
\begin{aligned}
\dot{\mathrm{B}} & =\dot{\mathrm{T}} \wedge \mathrm{N} \wedge \gamma+\mathrm{T} \wedge \dot{\mathrm{N}} \wedge \gamma+\mathrm{T} \wedge \mathrm{N} \wedge \dot{\gamma} \\
& =\left(\frac{\overline{D T}}{d s}+\langle\dot{\mathrm{T}}, \gamma\rangle \gamma\right) \wedge \mathrm{N} \wedge \gamma+\mathrm{T} \wedge\left(\frac{\overline{D N}}{d s}+\langle\dot{\mathrm{N}}, \gamma\rangle \gamma\right) \wedge \gamma \\
& =\mathrm{T} \wedge \frac{\overline{D N}}{d s} \wedge \gamma .
\end{aligned}
$$

Temos então

$$
\frac{\bar{D} \mathrm{~B}}{d s}=\mathrm{T} \wedge \frac{\bar{D} \mathrm{~N}}{d s} \wedge \gamma
$$

isto é, $\frac{\overline{D B}}{d s}$ é ortogonal a $B, T$ e $\gamma$. Logo

$$
\frac{\bar{D} \mathrm{~B}}{d s}=-\tau(s) \mathrm{N}
$$

onde $\tau(s)$ é chamada torção de $\gamma$.

Das igualdades anteriores, segue-se que

$$
\begin{aligned}
\langle\mathrm{N}, \mathrm{T}\rangle=0 & \Rightarrow\left\langle\frac{\overline{D N}}{d s}, \mathrm{~T}\right\rangle+\left\langle\mathrm{N}, \frac{\overline{D T}}{d s}\right\rangle=0 \\
& \Rightarrow\left\langle\frac{\overline{D N}}{d s}, \mathrm{~T}\right\rangle=-\kappa
\end{aligned}
$$


e

$$
\begin{aligned}
\langle\mathrm{N}, \mathrm{B}\rangle=0 & \Rightarrow\left\langle\frac{\overline{D N}}{d s}, \mathrm{~B}\right\rangle+\left\langle\mathrm{N}, \frac{\bar{D} \mathrm{~B}}{d s}\right\rangle=0 \\
& \Rightarrow\left\langle\frac{\overline{D N}}{d s}, \mathrm{~B}\right\rangle=\tau
\end{aligned}
$$

Portanto,

$$
\frac{\overline{D N}}{d s}=-\kappa \mathrm{T}+\tau \mathrm{B}
$$

No caso $c=-1$, observando-se apenas que $\frac{\bar{D} \mathrm{~B}}{d s}=\dot{\mathrm{B}}+\langle\dot{\mathrm{B}}, \gamma\rangle \gamma$, temos que valem as mesmas expressões para $\frac{\overline{D N}}{d s}$ e $\frac{\overline{D B}}{d s}$.

Logo dada uma curva regular unitária $\operatorname{em} \mathbb{Q}^{3}(c)$ as seguintes fórmulas, chamadas fórmulas de Frenet, verificam-se

$$
\begin{aligned}
& \bar{\nabla}_{\mathrm{T}} \mathrm{T}=\kappa \mathrm{N}, \\
& \bar{\nabla}_{\mathrm{T}} \mathrm{N}=-\kappa \mathrm{T}+\tau \mathrm{B}, \\
& \bar{\nabla}_{\mathrm{T}} \mathrm{B}=-\tau \mathrm{N} .
\end{aligned}
$$

Os dois próximos lemas são facilmente verificados.

Lema 1.11 Seja $\gamma: I \subset \mathbb{R} \longrightarrow \mathbb{Q}^{3}(c)$ uma curva regular em $\mathbb{Q}^{3}(c)$ com velocidade unitária. As condições seguintes são equivalentes:

1. $\kappa \equiv c$

2. $\gamma$ é uma geodésica em $\mathbb{Q}^{3}(c)$.

Lema 1.12 Nas condições do lema anterior, suponha que $\kappa(s)>0$. São equivalentes:

1. $\tau \equiv 0$.

2. $\gamma$ é uma curva plana em $\mathbb{Q}^{3}(c)$, isto é, $\gamma(I)$ está contida em uma 2-subvariedade totalmente geodésica de $\mathbb{Q}^{3}(c)$.

Determinemos uma expressão para o cálculo explícito da curvatura e da torção de uma curva regular com velocidade unitária em $\mathbb{Q}^{3}(c)$. Segue-se por (1.3) que no caso $c=1$

$$
\kappa=|\ddot{\gamma}-\langle\ddot{\gamma}, \gamma\rangle \gamma|=|\ddot{\gamma}+\gamma|
$$


e no caso $c=-1$

$$
\kappa=|\ddot{\gamma}+\langle\ddot{\gamma}, \gamma\rangle \gamma|=|\ddot{\gamma}-\gamma|
$$

isto é,

$$
\kappa=|\ddot{\gamma}+c \gamma| \text {. }
$$

Além disso, por (1.4) e (1.5) temos que nos casos $c= \pm 1$ vale que

$$
\begin{aligned}
-\tau & =\left\langle\frac{\bar{D} \mathrm{~B}}{d s}, \mathrm{~N}\right\rangle \\
& =\left\langle\mathrm{T} \wedge \frac{\overline{D N}}{d s} \wedge \gamma, \mathrm{N}\right\rangle \\
& =\frac{1}{\kappa^{2}}\left\langle\mathrm{~T} \wedge \frac{D}{d s}(\ddot{\gamma}+c \gamma) \wedge \gamma,(\ddot{\gamma}+c \gamma)\right\rangle \\
& =\frac{1}{\kappa^{2}}\langle\dot{\gamma} \wedge \ddot{\gamma} \wedge \gamma, \ddot{\gamma}\rangle .
\end{aligned}
$$

Apenas para constar, lembremos que no caso $\mathbb{R}^{3}$, vale que

$$
\kappa=|\ddot{\gamma}|, \quad \tau=\langle\dot{B}, N\rangle
$$

onde $T=\dot{\gamma}, \dot{T}=\kappa N$ e $B=T \times N$.

\subsection{Superfícies helicoidais em formas espaciais}

Geometricamente, um movimento helicoidal em $\mathbb{Q}^{3}(c)$ é a composição de um movimento de rotação com um de translação, no sentido que passamos a descrever.

Uma translação de passo $h$ em $\mathbb{Q}^{3}(c)$ é uma isometria $\mathcal{T}_{h}$ de $\mathbb{Q}^{3}(c)$ que deixa invariante uma geodésica $\gamma$, isto é, se $p \in \gamma$, então $\mathcal{T}_{h}(p) \in \gamma$ e $d\left(p, \mathcal{T}_{h}(p)\right)=h$, onde $d$ é a distância riemanniana de $\mathbb{Q}^{3}(c)$.

Uma rotação de ângulo $k$ ao redor de uma geodésica $\gamma$ é a isometria $\mathcal{R}_{k}$ de $\mathbb{Q}^{3}(c)$ que fixa ponto a ponto a geodésica $\gamma$ e se $p \in \mathcal{P}^{2}$, onde $\mathcal{P}^{2}$ é um plano totalmente geodésico em $\mathbb{Q}^{3}(c)$ ortogonal a $\gamma$, então $\mathcal{R}_{k}(p) \in \mathcal{P}^{2}$ e o ângulo entre as geodésicas que passam por $p$ e $q$ e por $\mathcal{R}_{k}(p)$ e $q$ é $k$, onde $q=\gamma \cap \mathcal{P}^{2}$.

Fixemos uma geodésica $\gamma$ em $\mathbb{Q}^{3}(c)$ e consideremos a translação $\mathcal{T}_{h}$ de passo $h$ ao longo de $\gamma$ e a rotação $\mathcal{R}_{k}$ de ângulo $k$ ao redor da mesma geodésica. A composta $\mathcal{H}_{\varphi}=\mathcal{T}_{h \varphi} \circ \mathcal{R}_{k \varphi}, \varphi \in \mathbb{R}$ é denominada movimento helicoidal ao redor do eixo $\gamma$ com passo $h$ e velocidade $k$.

Como queremos operar explicitamente com tais movimentos, é necessário conhecermos os subgrupos de isometrias a um parâmetro do grupo de isometrias de $\mathbb{Q}^{3}(c)$, ou movimentos rígidos do ambiente. 
Definição 1.13 Um subgrupo de isometrias a um parâmetro do grupo de isometrias de $\mathbb{Q}^{3}(c)$ é uma familia $\left\{\mathcal{H}_{\varphi}\right\}, \varphi \in \mathbb{R}$, onde para cada $\varphi$ fixo, $\mathcal{H}_{\varphi}$ é uma isometria de $\mathbb{Q}^{3}(c)$ e para quaisquer $\varphi, \lambda \in \mathbb{R}$, temos
1. $\mathcal{H}_{\varphi} \circ \mathcal{H}_{\lambda}=\mathcal{H}_{\varphi+\lambda}$,
2. $\mathcal{H}_{\varphi}^{-1}=\mathcal{H}_{-\varphi}$,
3. $\mathcal{H}_{0}=I d$.

Teorema 1.14 A menos de conjugação, os elementos dos subgrupos acima definidos têm a seguinte forma:

1. Caso $c=-1$ :

(a)

$$
\mathcal{H}_{\varphi}^{h, k}=\left(\begin{array}{cccc}
\cosh h \varphi & \sinh h \varphi & 0 & 0 \\
\sinh h \varphi & \cosh h \varphi & 0 & 0 \\
0 & 0 & \cos k \varphi & \sin k \varphi \\
0 & 0 & -\sin k \varphi & \cos k \varphi
\end{array}\right)
$$

(b)

$$
\mathcal{H}_{\varphi}^{h, 1}=\left(\begin{array}{cccc}
1+h^{2} \frac{\varphi^{2}}{2} & -h^{2} \frac{\varphi^{2}}{2} & h \varphi & 0 \\
h^{2} \frac{\varphi^{2}}{2} & 1-h^{2} \frac{\varphi^{2}}{2} & h \varphi & 0 \\
h \varphi & -h \varphi & 1 & 0 \\
0 & 0 & 0 & 1
\end{array}\right)
$$

2. Caso $c=0$;

$$
\mathcal{H}_{\varphi}^{h, k}=\left(\begin{array}{cccc}
\cos k \varphi & \sin k \varphi & 0 & 0 \\
-\sin k \varphi & \cos k \varphi & 0 & 0 \\
0 & 0 & 1 & h \varphi \\
0 & 0 & 0 & 1
\end{array}\right)
$$

3. Caso $c=1$ :

$$
\mathcal{H}_{\varphi}^{h, k}=\left(\begin{array}{cccc}
\cos h \varphi & \sin h \varphi & 0 & 0 \\
-\sin h \varphi & \cos h \varphi & 0 & 0 \\
0 & 0 & \cos k \varphi & \sin k \varphi \\
0 & 0 & -\sin k \varphi & \cos k \varphi
\end{array}\right)
$$


onde $h$ e $k$ são reais fixados $e \varphi \in \mathbb{R}$.

Demonstração:Ver [R]. Observamos que a conjugação mencionada é no sentido usual de conjugação de matrizes.

O movimento em (1.b) chama-se movimento parabólico, e em qualquer um dos outros casos, se $h=0$ o movimento é denominado rotacional, se $k=0$ é denominado translacional e, quando $h \neq 0$ e $k \neq 0$ é denominado helicoidal. Claramente temos que se $k \neq 0$, então $\left\{\mathcal{H}_{\varphi}^{h, k}: \varphi \in \mathbb{R}\right\}=\left\{\mathcal{H}_{\lambda}^{\frac{h}{k}, 1}: \lambda \in \mathbb{R}\right\}$. A constante $\frac{h}{k}$ é chamada passo do movimento helicoidal.

No que se segue não usaremos o movimento parabólico, e estaremos sempre supondo que $h \neq 0$ e $k \neq 0$. Para simplificarmos a notação usaremos $h$ ao invés de $\frac{h}{k} \mathrm{e}$ denotaremos $\mathcal{H}_{\varphi}:=\mathcal{H}_{\varphi}^{h, 1}$.

Observamos que os eixos (geodésicas) considerados no teorema anterior são:

$$
\begin{array}{ll}
(\cosh s, \sinh s, 0,0), & \text { se } c=-1 \text { (caso } 1 . a), \\
(0,0, s), & \text { se } c=0, \\
(\cos s, \sin s, 0,0), & \text { se } c=1 .
\end{array}
$$

Esses eixos serão chamados eixos invariantes pelo movimento helicoidal. Note ainda, que no caso $c=0$, estamos identificando $\mathbb{R}^{3}$ com o hiperplano $\left\{\left(x_{1}, x_{2}, x_{3}, 1\right)\right\}$ de $\mathbb{R}^{4}$.

Definição 1.15 Uma superfície $M^{2} \subset \mathbb{Q}^{3}(c)$ é dita helicoidal se permanece invariante por um movimento helicoidal, isto é, $\mathcal{H}_{\varphi}\left(M^{2}\right)=M^{2}, \forall \varphi \in \mathbb{R}$. Dizemos também que $M^{2}$ é $\left\{\mathcal{H}_{\varphi}\right\}_{\varphi \in R}-$ invariante.

Se $p \in \mathbb{Q}^{3}(c)$, a curva $\mathcal{H}_{\varphi}(p), \varphi \in \mathbb{R}$, é chamada de órbita de $p$ e está contida na superfície cujos pontos eqüidistam do eixo invariante pelo movimento helicoidal. $\mathrm{E}$ imediato que a órbita de um ponto é uma curva invariante pelo movimento helicoidal que a gerou, e vice versa, isto é, toda curva invariante por um movimento helicoidal é a órbita de um de seus pontos.

Se $M^{2} \subset \mathbb{Q}^{3}(c)$ é uma superfície helicoidal e $\mathcal{P}^{2}$ é um plano em $\mathbb{Q}^{3}(c)$, ortogonal ao eixo invariante pelo movimento, cálculos diretos mostram que a órbita de todo ponto $p \in M^{2}$ intercepta $\mathcal{P}^{2}$ ao menos uma vez (uma única vez quando $c \leq 0$ ). Temos então que toda superfície helicoidal é gerada por uma curva plana, isto é, $X(s, t)=\mathcal{H}_{s}(\alpha(t))$ é uma reparametrização de $M^{2}$, onde $\alpha(t)=M^{2} \cap \mathcal{P}^{2}$. A curva $\alpha(t)$ é chamada curva geratriz de $M^{2}$ e $\mathcal{P}^{2}$ o plano de órbitas. 
Segue-se do parágrafo anterior que conhecer a curva geratriz de uma superfície helicoidal pode ser interessante, já que podemos reduzir o estudo da superfície ao estudo da curva. Este método é conhecido como geometria equivariante. Na demonstração da próxima proposição damos um exemplo do uso da curva geratriz.

Proposição 1.16 Com a notação anterior, seja $M^{2} \subset \mathbb{Q}^{3}(c)$ uma superfície helicoidal reparametrizada por $X(s, t)=\mathcal{H}_{s}(\alpha(t))$. Então a curvatura média, $H$, e a curvatura Gaussiana, $K$, de $M^{2}$ são constantes em relação ao parâmetro s.

\section{Demonstração:}

Faremos a demonstração apenas para o caso $c=-1$, pois nos outros dois casos a demonstração segue-se análoga, guardadas as particularidades de cada ambiente.

A menos de isometria de $\mathbb{H}^{3}$, seja $\mathcal{P}^{2}=\left\{x \in \mathbb{H}^{3}: x_{2}=0\right\}$ a 2-subvariedade totalmente geodésica de $\mathbb{H}^{3}$ ortogonal ao eixo $\Gamma(r)=(\cosh r, \sinh r, 0,0)$ e seja $\alpha(t)=$ $\left(g_{1}(t), 0, g_{3}(t), g_{4}(t)\right)$ a curva geratriz da superfície, determinada pela intersecção da superfície com o plano $\mathcal{P}^{2}$. Temos então que

$$
\begin{gathered}
X(s, t)=\mathcal{H}_{s}(\alpha(t))=\left(g_{1} \cosh h s, g_{1} \sinh h s, g_{3} \cos s+\right. \\
\left.+g_{4} \sin s,-g_{3} \sin s+g_{4} \cos s\right)
\end{gathered}
$$

é uma reparametrização da superfície helicoidal.

Após cálculos simples, verifica-se que

$$
\begin{aligned}
\left|X_{s}\right|^{2} & =g_{1}^{2} h^{2}+g_{3}^{2}+g_{4}^{2} \\
\left|X_{t}\right|^{2} & =-\dot{g}_{1}^{2}+\dot{g}_{3}^{2}+\dot{g}_{4}^{2} \\
\left\langle X_{s}, X_{t}\right\rangle & =-g_{3} \dot{g}_{4}+g_{4} \dot{g}_{3} \\
\left\langle X_{s s}, X_{t}\right\rangle & =-g_{1} \dot{g}_{1} h^{2}-g_{3} \dot{g}_{3}-g_{4} \dot{g}_{4} \\
\left\langle X_{s s}, X_{t t}\right\rangle & =-g_{1} \ddot{g}_{1} h^{2}-g_{3} \ddot{g}_{3}-g_{4} \ddot{g}_{4} \\
\left\langle X_{s t}, X_{t t}\right\rangle & =\ddot{g}_{3} \dot{g}_{4}-\dot{g}_{3} \ddot{g}_{4} \\
\left\langle X_{s t}, X_{s t}\right\rangle & =\dot{g}_{1}^{2} h^{2}+\dot{g}_{3}^{2}+\dot{g}_{4}^{2} .
\end{aligned}
$$

Consideremos então a base ortonormal $\left\{\frac{X_{t}}{\left|X_{t}\right|}, \frac{\tilde{X}_{s}}{\left|\tilde{X}_{s}\right|}\right\}$, onde $\tilde{X}_{s}=X_{s}-\left\langle X_{s}, X_{t}\right\rangle \frac{X_{t}}{\left|X_{t}\right|^{2}}$, e calculemos $K\left(\frac{X_{t}}{\left|X_{t}\right|}, \frac{\tilde{X}_{s}}{\left|\tilde{X}_{s}\right|}\right)$ e $H\left(\frac{X_{t}}{\left|X_{t}\right|}, \frac{\tilde{X}_{s}}{\left|\tilde{X}_{s}\right|}\right)$. Usando-se a expressão para o cálculo da curvatura Gaussiana e da curvatura média de uma superfície em $\mathbb{H}^{3}$ segue-se, após vários cálculos, que

$$
K=-1+\frac{1}{\left|X_{t}\right|^{4}\left|\tilde{X}_{s}\right|^{4}}\left(\left\langle\alpha\left(X_{t}, X_{t}\right), \alpha\left(\tilde{X}_{s}, \tilde{X}_{s}\right)\right\rangle-\left|\alpha\left(X_{t}, \tilde{X}_{s}\right)\right|^{2}\right)
$$


e

$$
H=\frac{1}{4}\left(\frac{\left|\alpha\left(X_{t}, X_{t}\right)\right|^{2}}{\left|X_{t}\right|^{4}}+\frac{\left|\alpha\left(\tilde{X}_{s}, \tilde{X}_{s}\right)\right|^{2}}{\left|\tilde{X}_{s}\right|^{4}}+\frac{2\left\langle\alpha\left(X_{t}, X_{t}\right), \alpha\left(\tilde{X}_{s}, \tilde{X}_{s}\right)\right\rangle}{\left|\tilde{X}_{s}\right|^{2}\left|X_{t}\right|^{2}}\right),
$$

onde

$$
\begin{aligned}
& \left|\alpha\left(X_{t}, \tilde{X}_{s}\right)\right|^{2}=\left|\alpha\left(X_{t}, X_{s}\right)\right|^{2}-2 \frac{\left\langle X_{s}, X_{t}\right\rangle}{\left|X_{t}\right|^{2}}\left\langle\alpha\left(X_{s}, X_{t}\right), \alpha\left(X_{t}, X_{t}\right)\right\rangle+ \\
& +\frac{\left\langle X_{s}, X_{t}\right\rangle^{2}}{\left|X_{t}\right|^{4}}\left|\alpha\left(X_{t}, X_{t}\right)\right|^{2} \\
& \left|\alpha\left(X_{t}, X_{s}\right)\right|^{2}=\left|X_{s t}\right|^{2}+\left\langle X_{s t}, X\right\rangle^{2}-\frac{\left\langle X_{s t}, X_{t}\right\rangle^{2}}{\left|X_{t}\right|^{2}}+ \\
& -\frac{1}{\left|\tilde{X}_{s}\right|^{2}}\left(\left\langle X_{s t}, X_{s}\right\rangle-\frac{\left\langle X_{t}, X_{s}\right\rangle\left\langle X_{s t}, X_{t}\right\rangle}{\left|X_{t}\right|^{2}}\right)^{2} \\
& \left|\alpha\left(X_{t}, X_{t}\right)\right|^{2}=\left|X_{t t}\right|^{2}+\left\langle X_{t t}, X\right\rangle^{2}-\frac{\left\langle X_{t t}, X_{t}\right\rangle^{2}}{\left|X_{t}\right|^{2}}-\frac{\left\langle X_{t t}, \tilde{X}_{s}\right\rangle^{2}}{\left|\tilde{X}_{s}\right|^{2}} \\
& \left|\alpha\left(\tilde{X}_{s}, \tilde{X}_{s}\right)\right|^{2}=\left|\alpha\left(X_{s}, X_{s}\right)\right|^{2}+\frac{4\left\langle X_{t}, X_{s}\right\rangle^{2}}{\left|X_{t}\right|^{4}}\left|\alpha\left(X_{s}, X_{t}\right)\right|^{2}+ \\
& +\frac{\left\langle X_{t}, X_{s}\right\rangle^{4}}{\left|X_{t}\right|^{8}}\left|\alpha\left(X_{t}, X_{t}\right)\right|^{2}+ \\
& -\frac{4\left\langle X_{t}, X_{s}\right\rangle}{\left|X_{t}\right|^{2}}\left\langle\alpha\left(X_{s}, X_{t}\right), \alpha\left(X_{s}, X_{s}\right)\right\rangle+ \\
& +\frac{2\left(X_{t}, X_{s}\right)^{2}}{\left|X_{t}\right|^{4}}\left\langle\alpha\left(X_{t}, X_{t}\right), \alpha\left(X_{s}, X_{s}\right)\right\rangle+ \\
& -\frac{4\left\langle X_{t}, X_{s}\right)^{3}}{\left|X_{t}\right|^{6}}\left\langle\alpha\left(X_{s}, X_{t}\right), \alpha\left(X_{t}, X_{t}\right)\right\rangle \text {, } \\
& \left|\alpha\left(X_{s}, X_{s}\right)\right|^{2}=\left|X_{s s}\right|^{2}+\left\langle X_{s s}, X\right\rangle^{2}-\frac{\left\langle X_{s s}, X_{t}\right\rangle^{2}}{\left|X_{t}\right|^{2}}+ \\
& -\frac{1}{\left|\tilde{X}_{s}\right|^{2}}\left(\left\langle X_{s s}, X_{s}\right\rangle-\frac{\left\langle X_{t}, X_{s}\right\rangle\left\langle X_{s s}, X_{t}\right\rangle}{\left|X_{t}\right|^{2}}\right)^{2} \\
& \left\langle\alpha\left(X_{s}, X_{t}\right), \alpha\left(X_{t}, X_{t}\right)\right\rangle=\left\langle X_{s t}, X_{t t}\right\rangle+\left\langle X_{t t}, X\right\rangle\left\langle X_{s t}, X\right\rangle-\frac{\left\langle X_{t t}, X_{t}\right\rangle\left\langle X_{s t}, X_{t}\right\rangle}{\left|X_{t}\right|^{2}}+ \\
& -\frac{1}{\left|\tilde{X}_{s}\right|^{2}}\left(\left\langle X_{t t}, X_{s}\right\rangle-\frac{\left\langle X_{t}, X_{s}\right\rangle\left\langle X_{t t}, X_{t}\right\rangle}{\left|X_{t}\right|^{2}}\right) \\
& \left(\left\langle X_{t s}, X_{s}\right\rangle-\frac{\left\langle X_{t}, X_{s}\right\rangle\left\langle X_{s t}, X_{t}\right\rangle}{\left|X_{t}\right|^{2}}\right) \\
& \left\langle\alpha\left(\tilde{X}_{s}, \tilde{X}_{s}\right), \alpha\left(X_{t}, X_{t}\right)\right\rangle=\left\langle\alpha\left(X_{s}, X_{s}\right), \alpha\left(X_{t}, X_{t}\right)\right\rangle+ \\
& -2 \frac{\left\langle X_{t}, X_{s}\right\rangle}{\left|X_{t}\right|^{2}}\left\langle\alpha\left(X_{s}, X_{t}\right), \alpha\left(X_{t}, X_{t}\right)\right\rangle+ \\
& +\frac{\left\langle X_{t}, X_{s}\right\rangle^{2}}{\left|X_{t}\right|^{4}}\left|\alpha\left(X_{t}, X_{t}\right)\right|^{2} \\
& \left\langle\alpha\left(X_{s}, X_{s}\right), \alpha\left(X_{t}, X_{t}\right)\right\rangle=\left\langle X_{s s}, X_{t t}\right\rangle+\left\langle X_{s s}, X\right\rangle\left\langle X_{t t}, X\right\rangle-\frac{\left\langle X_{t t}, X_{t}\right\rangle\left\langle X_{s s}, X_{t}\right\rangle}{\left|X_{t}\right|^{2}}+ \\
& -\frac{1}{\left|\tilde{X}_{s}\right|^{2}}\left(\left\langle X_{s s}, X_{s}\right\rangle-\frac{\left\langle X_{t}, X_{s}\right\rangle\left\langle X_{s s}, X_{t}\right\rangle}{\left|X_{t}\right|^{2}}\right) \\
& \left(\left\langle X_{t t}, X_{s}\right\rangle-\frac{\left\langle X_{t}, X_{s}\right\rangle\left\langle X_{t t}, X_{t}\right\rangle}{\left|X_{t}\right|^{2}}\right) \\
& \left\langle\alpha\left(X_{s}, X_{s}\right), \alpha\left(X_{s}, X_{t}\right)\right\rangle=\left\langle X_{s s}, X_{s t}\right\rangle+\left\langle X_{s t}, X\right\rangle\left\langle X_{s s}, X\right\rangle-\frac{\left\langle X_{s t}, X_{t}\right\rangle\left\langle X_{s s}, X_{t}\right\rangle}{\left|X_{t}\right|^{2}}+ \\
& -\frac{1}{\left|\tilde{X}_{s}\right|^{2}}\left(\left\langle X_{s t}, X_{s}\right\rangle-\frac{\left\langle X_{t}, X_{s}\right\rangle\left\langle X_{s t}, X_{t}\right\rangle}{\left|X_{t}\right|^{2}}\right) \\
& \left(\left\langle X_{s s}, X_{s}\right\rangle-\frac{\left\langle X_{t}, X_{s}\right\rangle\left\langle X_{s s}, X_{t}\right\rangle}{\left|X_{t}\right|^{2}}\right)
\end{aligned}
$$


Usando-se agora (1.9) não é difícil verificar que $K$ e $H$ dependem apenas da variável $t$, isto é, são constantes em relação a variável $s$.

Para finalizar esta seção faremos uma observação que será muito importante no capítulo 3:

Observação 1.17 Duas superfícies $M_{1}, M_{2}$ em $\mathbb{Q}^{3}(c)$ são ditas congruentes quando existe uma isometria $g \in I S O\left(\mathbb{Q}^{3}(c)\right)$ tal que $g\left(M_{1}\right)=M_{2}$; dois subgrupos $\mathrm{H}_{1}, \mathrm{H}_{2}$ de $I S O\left(\mathbb{Q}^{3}(c)\right)$ são ditos conjugados quando existe $g \in I S O\left(\mathbb{Q}^{3}(c)\right)$ tal que $\mathrm{H}_{1}=$ $g \circ \mathrm{H}_{2} \circ g^{-1}$.

Segue-se então da definição (1.15) e desta observação que se $M_{1}$ é $H_{1}$-invariante e congruente a $M_{2}$, então existe $H_{2}$ conjugado a $H_{1}$ tal que $M_{2}$ é $H_{2}$-invariante. De fato, como $M_{1}$ é congruente a $M_{2}$ existe $g \in I S O\left(\mathbb{Q}^{3}(c)\right)$ tal que $g\left(M_{2}\right)=M_{1}$, tomemos $H_{2}=g^{-1} \circ H_{1} \circ g$. É claro que $H_{1}$ e $H_{2}$ são conjugados e além disso $M_{2}$ é $H_{2}$-invariante, pois se $h_{2} \in H_{2}$ então $h_{2}\left(M_{2}\right)=h_{2}\left(g^{-1}\left(M_{1}\right)\right)=g^{-1} \circ\left(g \circ h_{2} \circ g^{-1}\right)\left(M_{1}\right)=g^{-1}\left(M_{1}\right)=$ $M_{2}$, já que $\left(g \circ h_{2} \circ g^{-1}\right) \in H_{1}$ e $M_{1}$ é $H_{1}$-invariante.

\subsection{Subvariedades regradas em formas espaciais}

Sejam $\mathbb{Q}^{m}(c)$ e $M^{n+1}$ uma subvariedade $(n+1)$-dimensional de $\mathbb{Q}^{m}(c)$. Dizemos que $M^{n+1}$ é uma subvariedade regrada de $\mathbb{Q}^{m}(c)$ se existe uma folheação $n$-dimensional de $M^{n+1}$ por subvariedades totalmente geodésicas de $\mathbb{Q}^{m}(c)$, isto é, dado $p \in M^{n+1}$ e $\mathcal{F}_{p}$ a folha da folheação passando por $p$, então $\mathcal{F}_{p}$ é uma subvariedade totalmente geodésica de $\mathbb{Q}^{m}(c)$ de dimensão $n$.

O campo de direções normais à folheação, em $M^{n+1}$, é localmente integrável. Seja então $\gamma=\gamma(s): I \in \mathbb{R} \longrightarrow M^{n+1}$ uma curva integral do campo normal à folheação, onde $s$ é o parâmetro comprimento de arco. Sejam ainda $e_{1}(s), e_{2}(s), \ldots e_{n}(s) n$ campos ortonormais ao longo de $\gamma(s)$, que geram o espaço tangente à folha ao longo de $\gamma(s)$ para cada $s$. Denotando por $\langle$,$\rangle a métrica do espaço ambiente, \mathbb{R}^{m+1}$ ou $\mathbb{L}^{m+1}$, podemos escolher $e_{1}(s), e_{2}(s), \ldots e_{n}(s)$ de forma que $\left\langle e_{j}, \dot{e}_{i}\right\rangle=0$ para todo $1 \leq i, j \leq n$, onde '.' é a derivação usual em relação à variável $s$ no espaço ambiente. A afirmação anterior segue-se do próximo lema, cuja demonstração pode ser encontrada em [BDJ]. 
Lema 1.18 Dadas uma curva $\gamma$ em uma variedade Riemanniana $N^{m}$ e $n$ campos ortonormais $e_{1}(s), e_{2}(s), \ldots e_{n}(s)$ tangentes a $N$ ao longo de $\gamma$, com $n<m$, sempre podemos escolher campos ortonormais $f_{1}(s), f_{2}(s), \ldots f_{n}(s)$, ao longo de $\gamma$ tais que

1. os conjuntos $\left\{f_{i}(s), 1 \leq i \leq n\right\}$ e $\left\{e_{i}(s), 1 \leq i \leq n\right\}$ geram o mesmo subespaço de $T_{\gamma(s)} N$;

2. o campo de vetores $\frac{\bar{D}}{d s} f_{i}(s)$ é normal ao subespaço gerado por $\left\{f_{j}(s), 1 \leq j \leq n\right\}$, para todo $1 \leq i \leq n$, onde $\frac{\bar{D}}{d s}$ é a derivada covariante em $N$.

Temos então que uma subvariedade regrada $M^{n+1}$ pode ser localmente parametrizada a partir da curva integral $\gamma$ e dos $n$ campos ortonormais $e_{1}(s), e_{2}(s), \ldots e_{n}(s)$, isto é, $M^{n+1}$ pode ser localmente parametrizada por

$$
X\left(s, t_{1}, \ldots \ldots, t_{n}\right)=\exp _{\gamma(s)}\left(\sum_{i=1}^{i=n} t_{i} e_{i}(s)\right)
$$

onde exp é a aplicação exponencial de $\mathbb{Q}^{m}(c)$, que pode ser expressa por

$$
\exp _{p}(r v)=f(r) p+g(r) v
$$

onde $p \in \mathbb{Q}^{m}(c), v \in T_{p} \mathbb{Q}^{m}(c)$ com $\|v\|=1$ e as funções $f$ e $g$ são dadas por

$$
\left\{\begin{array}{lll}
f(r)=1, & g(r)=r, & \text { se } c=0 \\
f(r)=\cos r, & g(r)=\sin r, & \text { se } c=1 \\
f(r)=\cosh r, & g(r)=\sinh r, & \text { se } c=-1
\end{array}\right.
$$

Utilizando-se (1.11) podemos reescrever (1.10) de uma forma mais simples. De fato,

$$
\begin{aligned}
& X\left(s, t_{1}, \ldots \ldots, t_{n}\right)=\exp _{\gamma(s)}\left(\sum_{i=1}^{i=n} t_{i} e_{i}(s)\right)= \\
& =\exp _{\gamma(s)} r\left(t_{1}, \ldots \ldots t_{n}\right)\left(\frac{\sum_{i=1}^{i=n} t_{i} e_{i}(s)}{r\left(t_{1}, \ldots \ldots t_{n}\right)}\right),
\end{aligned}
$$

onde $r\left(t_{1}, \ldots \ldots . t_{n}\right)=\sqrt{\sum_{i=1}^{i=n} t_{i}^{2}}$. Portanto

$$
X\left(s, t_{1}, \ldots \ldots, t_{n}\right)=f\left(r\left(t_{1}, \ldots . t_{n}\right)\right) \gamma(s)+\frac{g\left(r\left(t_{1}, \ldots . t_{n}\right)\right)}{r\left(t_{1}, \ldots . . t_{n}\right)} \sum_{i=1}^{i=n} t_{i} e_{i}(s) .
$$


Denotando $\gamma(s)=e_{0}(s), \varphi_{0}\left(t_{1}, \ldots . t_{n}\right)=f\left(r\left(t_{1}, \ldots . . t_{n}\right)\right)$ e $\varphi_{j}\left(t_{1}, \ldots . t_{n}\right)=\frac{g\left(r\left(t_{1}, \ldots . . t_{n}\right)\right)}{r\left(t_{1}, \ldots . . t_{n}\right)} t_{j}$ $1 \leq j \leq n$, podemos reescrever a última igualdade como

$$
X\left(s, t_{1}, \ldots \ldots, t_{n}\right)=\sum_{i=0}^{i=n} \varphi_{i}\left(t_{1}, \ldots \ldots, t_{n}\right) e_{i}(s) .
$$

Definição 1.19 O parâmetro s, quando usado no sentido anterior, será chamado de parâmetro "translacional".

A parametrização dada em (1.13) tem a característica que os vetores $X_{t_{j}}, 1 \leq j \leq$ $n$, são tangentes a subvariedade totalmente geodésica de $\mathbb{Q}^{m}(c)$, ou seja, se $\alpha$ denota a segunda forma fundamental da subvariedade $M^{n+1}$, temos que $\alpha\left(X_{t_{j}}, X_{t_{k}}\right)=0$, $1 \leq j, k \leq n$.

\subsection{Superfícies regradas no espaço de De Sitter}

De forma análoga ao caso Riemanniano, podemos definir no espaço de De Sitter a noção de superfície regrada, isto é, dizemos que $M^{2}$ é uma superfície regrada em $\mathbb{S}_{1}^{3}$, se $M^{2}$ é folheada por geodésicas de $\mathbb{S}_{1}^{3}$, ou seja, se por cada ponto de $M^{2}$ passa uma geodésica de $\mathbb{S}_{1}^{3}$. Como o espaço de De Sitter admite três tipos de geodésicas, podemos definir três tipos de superfícies regradas:

1. Superfície regrada por geodésicas tipo espaço, que podem se localmente parametrizadas como

$$
X(s, t)=e_{0}(s) \cos t+e_{1}(s) \sin t
$$

onde $e_{0}$ é uma curva na superfície e $e_{1}$ é um vetor tangente à superfície ao longo de $e_{0}$, tal que $\left\langle e_{1}, e_{1}\right\rangle=1$.

2. Superfície regrada por geodésicas tipo tempo, que podem se localmente parametrizadas como

$$
X(s, t)=e_{0}(s) \cosh t+e_{1}(s) \sinh t
$$

onde $e_{0}$ é uma curva na superfície e $e_{1}$ é um vetor tangente à superfície ao longo de $e_{0}$, tal que $\left\langle e_{1}, e_{1}\right\rangle=-1$.

3. Superfície regrada por geodésicas tipo luz, que podem se localmente parametrizadas como

$$
X(s, t)=e_{0}(s)+e_{1}(s) t
$$


onde $e_{0}$ é uma curva na superfície e $e_{1}$ é um vetor tipo luz tangente à superfície ao longo de $e_{0}$.

Nos próximos capítulos, nos resultados de $\mathbb{S}_{\lambda}^{n+1}$, consideraremos apenas as superfícies regradas por geodésicas tipo espaço. E importante observar, neste caso, que o tipo causal da folha não define o tipo causal da superfície. Se analisarmos as superfícies

$$
X(s, t)=\left(\sinh s \cos t, \cosh s \cos t, \frac{1}{\sqrt{2}} \sin t, \frac{1}{\sqrt{2}} \sin t\right)
$$

e

$$
\begin{gathered}
Y(s, t)=\left(\cosh (\sqrt{2} s) \cos t+\frac{1}{\sqrt{2}} \sinh (\sqrt{2} s) \sin t, \sinh (\sqrt{2} s) \cos t+\frac{1}{\sqrt{2}} \cosh (\sqrt{2} s) \sin t,\right. \\
\left.\sqrt{2} \sin (2 s) \cos t+\frac{1}{\sqrt{2}} \cos (2 s) \sin t,-\sqrt{2} \cos (2 s) \cos t+\frac{1}{\sqrt{2}} \sin (2 s) \sin t\right)
\end{gathered}
$$

é fácil verificar que

$$
\left\langle X_{s}, X_{s}\right\rangle=-\cos ^{2} t, \quad\left\langle X_{t}, X_{t}\right\rangle=1
$$

$\mathrm{e}$

$$
\left\langle Y_{s}, Y_{s}\right\rangle=10 \cos ^{2} t+\sin ^{2} t, \quad\left\langle Y_{t}, Y_{t}\right\rangle=1
$$

donde segue-se que $X(s, t)$ define uma superfície tipo tempo e $Y(s, t)$ uma superfície tipo espaço, ambas folheadas por geodésicas tipo espaço. Note, no entanto, que se tomarmos uma superfície tipo espaço regrada, ela é necessariamente folheada por geodésicas tipo espaço.

\subsection{Hipersuperfícies de Weingarten}

Definição 1.20 Uma hipersuperfície conexa em $\mathbb{Q}^{n+1}(c)$ é chamada de hipersuperfície de Weingarten ou de $W$-hipersuperfície, se as suas curvaturas escalar $S$ e média $H$ satisfazem uma relação não trivial, isto é, se existe uma função diferenciável não constante $F$ tal que $F(H, S)=0$.

Quando $n=2$, trocamos na definição anterior, a curvatura escalar pela curvatura Gaussiana, K, já que neste caso estas curvaturas coincidem. Analisando a definição anterior para os casos $n=2$ e $n \geq 3$ segue-se uma caracterização das superfícies e 
hipersuperfícies de Weingarten, que será muito importante no desenvolvimento do Capítulo 3 e 5 , respectivamente.

Denotando-se por $H_{s}=\frac{\partial H}{\partial s}, H_{t}=\frac{\partial H}{\partial t}, K_{s}=\frac{\partial K}{\partial s}$ e $K_{t}=\frac{\partial K}{\partial t}$ temos, no caso $n=2$, o seguinte resultado:

Proposição 1.21 Seja $X(s, t)$ a parametrização de uma superfície conexa em $\mathbb{Q}^{3}(c)$. $X(s, t)$ parametriza uma superficie de Weingarten se, $e$ somente se, os gradientes de $H$ e $K$ são linearmente dependentes, ou em termos das derivadas parciais, se $H_{s} K_{t}-H_{t} K_{s}=0$.

Segue-se então, pela proposição anterior, que para determinarmos quais são as superfícies de Weingarten em $\mathbb{Q}^{3}(c)$ basta verificarmos quais são as superfícies que satisfazem a relação $H_{s} K_{t}=H_{t} K_{s}$. Exemplos triviais de superfícies de Weingarten são as superfícies com curvatura média constante, com curvatura Gaussiana constante e com curvatura média e Gaussiana constantes em relação a um dos parâmetros. Sobre esta última classe de exemplos, temos o seguinte resultado:

Proposição 1.22 Seja $X(s, t)$ a parametrização de uma superfície conexa de Weingarten tal que suas curvaturas média e Gaussiana não sejam constantes. Então a curvatura média da superfície é constante em relação a um dos parâmetros se, e somente se, a curvatura Gaussiana também o for.

\section{Demonstração:}

Suponhamos, sem perda de generalidade, que a curvatura média da superfície seja constante em relação ao parâmetro $t$. Temos então que a superfície verifica a igualdade $H_{s} K_{t}=0$.

Se existe um ponto $p_{0} \in M^{2}$ tal que $H_{s}\left(p_{o}\right) \neq 0$, então existe uma vizinhança $V \ni p_{o}$ tal que $H_{s}(q) \neq 0, \forall q \in V$. Logo $K_{t}(q)=0, \forall q \in V$, donde segue-se que em $V$ vale o desejado.

Consideremos agora $Z\left(H_{s}\right)$ o conjunto dos zeros da função $H_{s}$. Como $H$ não é função constante, segue-se que $Z\left(H_{s}\right) \varsubsetneqq M^{2}$, e portanto existe um ponto $p_{o} \in$ $\partial\left(Z\left(H_{s}\right)\right)$. Logo existe uma seqüência $\left\{s_{n}\right\}_{n \in \mathbb{N}} \notin Z\left(H_{s}\right), \forall n$, convergindo para $p_{o}$, donde segue-se então que $K_{t}\left(s_{n}\right)=0, \forall n$, e portanto, $K_{t}\left(p_{o}\right)=0$.

No caso $n \geq 3$, usando-se a notação da Definição (1.20), segue-se que se calcularmos a derivada exterior de $F$ teremos que

$$
\frac{\partial F}{\partial H} d H+\frac{\partial F}{\partial S} d S=0
$$


donde segue-se que

$$
\frac{\partial F}{\partial H} d H \wedge d S=0, \quad \frac{\partial F}{\partial S} d H \wedge d S=0
$$

e portanto

$$
d H \wedge d S=0
$$

pois as derivadas parciais de $F$ não se anulam simultaneamente.

\subsection{Redução de codimensão}

Dizemos que uma imersão isométrica $f: M^{n} \rightarrow \tilde{M}^{n+p}$ admite redução de codimensão para $q$ se existe uma subvariedade totalmente geodésica $\tilde{M}^{n+q}$ em $\tilde{M}^{n+p}, \operatorname{com} q<p$, tal que $f\left(M^{n}\right) \subset \tilde{M}^{n+q}$. O fato de podermos reduzir a codimensão de imersões isométricas será fundamental para o desenvolvimento do capítulo 5. A seguir enunciamos, sem demonstração, uma versão do teorema de redução de codimensão no caso de imersões isométricas em espaço forma, ver [E], [D]. Depois demonstraremos uma versão deste teorema no caso de uma imersão isométrica de uma variedade Riemanniana em $\mathbb{L}^{n+p}$. Não encontramos referência sobre este resultado, apesar de acharmos que ele é bem conhecido. Comecemos dando alguns conceitos básicos.

Dada uma imersão isométrica $f: M^{n} \rightarrow \tilde{M}^{n+p}$, chamamos de primeiro espaço normal da imersão $f$ em $x \in M$ o subespaço $N_{1}(x) \subset T_{x} M^{\perp}$ gerado pela segunda forma fundamental, $\alpha$, da imersão $f$, isto é,

$$
N_{1}(x)=\operatorname{span}\left\{\alpha(X, Y): X, Y \in T_{x} M\right\},
$$

ou equivalentemente,

$$
N_{1}(x)=\left\{\xi \in T_{x} M^{\perp}: A_{\xi}=0\right\}^{\perp} .
$$

A imersão $f$ é chamada de 1-regular se a dimensão de $N_{1}$ é constante ao longo de $M$, e é chamada de substancial se a codimensão de $f$ não pode ser reduzida. A menor codimensão à qual a imersão $f$ pode ser reduzida, é chamada de codimensão substancial.

Teorema 1.23 Seja $f: M^{n} \rightarrow \mathbb{Q}^{n+p}(c)$ uma imersão isométrica de uma variedade Riemanniana em $\mathbb{Q}^{n+p}(c)$. Suponha que existe um subfibrado paralelo $L$ do fibrado normal, de posto $q<p$, tal que $N_{1}(x) \subset L(x)$ para todo $x \in M^{n}$. Então podemos reduzir a codimensão da imersão $f$ para $q$. 
Corolário 1.24 Seja $f: M^{n} \rightarrow \mathbb{Q}^{n+p}(c)$ uma imersão isométrica 1-regular de uma variedade Riemanniana em $\mathbb{Q}^{n+p}(c)$. Se $N_{1}$ é um subfibrado paralelo de posto $q<p$, então $f$ tem codimensão substancial $q$.

Teorema 1.25 Seja $f: M^{n} \rightarrow \mathbb{L}^{n+p}$ uma imersão isométrica de uma variedade Riemanniana conexa em $\mathbb{L}^{n+p}$. Suponha que existe um subfibrado paralelo não degenerado $L$ do fibrado normal, de posto $q<p$, tal que $N_{1}(x) \subset L(x)$ para todo $x \in M^{n}$. Então podemos reduzir a codimensão da imersão $f$ para $q$.

\section{Demonstração:}

Como $M^{n}$ é uma variedade Riemanniana, a métrica restrita ao espaço tangente a $M^{n}$ em $x$ é positiva definida, donde segue-se que $T_{x} \mathbb{L}^{n+p}=T_{x} M \oplus T_{x} M^{\perp}, \forall x \in M^{n}$, onde $T_{x} M^{\perp}$ é um subespaço tipo tempo.

Observamos, em primeiro lugar, que segue-se do fato de $T_{x} M^{\perp}$ ser um subespaço tipo tempo que $L(x) \subset T_{x} M^{\perp}$ pode ser um subespaço tipo tempo ou tipo espaço, já que por hipótese $L(x)$ é não degenerado. Em ambos os casos temos que $T_{x} M^{\perp}=$ $L(x) \oplus L^{\perp}(x), \operatorname{com} L^{\perp}(x)$ tipo espaço ou tipo tempo, respectivamente.

É fácil verificar, nestes dois casos, que o subfibrado $L^{\perp}$ é paralelo. De fato, se $\xi$ e $\eta$ são campos em $L^{\perp}$ e $L$, respectivamente, temos

$$
\begin{aligned}
X\langle\xi, \eta\rangle=0, \forall X \in \chi(M) & \Rightarrow\left\langle\nabla \frac{1}{X} \xi, \eta\right\rangle+\left\langle\xi, \nabla_{X}^{\frac{1}{X}} \eta\right\rangle=0 \\
& \Rightarrow\left\langle\nabla_{X}^{\frac{1}{X}} \xi, \eta\right\rangle=0,
\end{aligned}
$$

logo $\nabla_{X}^{\frac{1}{X}} \xi$ é um campo em $L^{\perp}$, pois $L$ é não degenerado.

Sejam $x_{0} \in M$ um ponto arbitrário, $\gamma: I \rightarrow M$ uma curva arbitrária passando por $x_{0}, \eta$ um vetor em $L^{\perp}\left(x_{0}\right)$ e $\eta_{t}$ o transporte paralelo de $\eta$ ao longo de $\gamma$ na conexão normal. Como $L^{\perp}$ é paralelo, temos que $\eta_{t} \in L^{\perp}(\gamma(t)), \forall t \in I$. Segue-se então, pela fórmula de Weingarten e do fato que $N_{1} \subset L$, que

$$
\frac{d \eta_{t}}{d t}(t)=\bar{\nabla}_{\dot{\gamma}(t)} \eta_{t}=-A_{\eta_{t}} \dot{\gamma}(t)+\nabla_{\dot{\gamma}(t)}^{\perp} \eta_{t}=0
$$

onde $\bar{\nabla}$ é a conexão de Levi Civita de $\mathbb{L}^{n+p}$ e $\nabla^{\perp}$ é a conexão normal de $M^{n}$. Portanto $\eta_{t}=\eta$ é um vetor constante em $\mathbb{L}^{n+p}$.

Segue assim que

$$
\frac{d}{d t}\left\langle f(\gamma(t))-f\left(x_{0}\right), \eta\right\rangle=\left\langle f_{*}(\dot{\gamma}(t)), \eta\right\rangle=0,
$$

e portanto $\left\langle f(\gamma(t))-f\left(x_{0}\right), \eta\right\rangle=$ cte. 
Como $\gamma$ e $\eta$ são arbitrários, temos então que

$$
f(M) \subset E\left(x_{0}\right)
$$

onde $E\left(x_{0}\right)=T_{x_{0}} M \oplus L\left(x_{0}\right)$ é uma subvariedade totalmente geodésica $(n+q)$ dimensional de $\mathbb{L}^{n+p}$. Observe que $\mathbb{E}\left(x_{0}\right)$ é isométrico a $\mathbb{R}^{n+q}$ ou a $\mathbb{L}^{n+q}$ conforme $L\left(x_{0}\right)$ seja um subespaço tipo espaço ou tipo tempo, respectivamente.

Logo a codimensão da imersão $f$ pode ser reduzida.

Corolário 1.26 Seja $f: M^{n} \rightarrow \mathbb{L}^{n+p}$ uma imersão isométrica 1-regular de uma variedade Riemanniana conexa em $\mathbb{L}^{n+p}$. Se $N_{1}$ é um subfibrado paralelo não degenerado, de posto $q<p$, então $f$ tem codimensão substancial $q$.

Corolário 1.27 Seja $f: M^{n} \rightarrow \mathbb{S}_{1}^{n+p}$ uma imersão isométrica 1-regular de uma variedade conexa tipo espaço no espaço de De Sitter. Se $N_{1}$ é um subfibrado paralelo $n \tilde{a} o$ degenerado, de posto $q<p$, então $f$ tem codimensão substancial $q$.

\section{Demonstração:}

Consideremos a imersão isométrica $\tilde{f}: M^{n} \rightarrow \mathbb{L}^{n+p+1}$ dada por $\tilde{f}=i \circ f$, onde $i: \mathbb{S}_{1}^{n+p} \rightarrow \mathbb{L}^{n+p+1}$ é a inclusão canônica do espaço de De Sitter no espaço de Lorentz.

É imediato que $\tilde{T}_{x} M^{\perp}=T_{x} M^{\perp} \oplus \operatorname{span}\{f(x)\}, \forall x \in M$. Além disso, observando-se que $\tilde{T}_{x} M=T_{x} M, \forall x \in M$, segue-se que

$$
\tilde{N}_{1}(x) \subset N_{1}(x) \oplus \operatorname{span}\{f(x)\} \text {. }
$$

Mostremos que o fibrado $\tilde{L}$ com fibra $\tilde{L}(x)=N_{1}(x) \oplus \operatorname{span}\{f(x)\}$, é paralelo.

Como $M^{n}$ é uma subvariedade tipo espaço, temos que $T_{x} M$ é um subespaço riemanniano e portanto $T_{x} M^{\perp}$ é um subespaço tipo tempo. Segue-se então do fato de $N_{1}(x) \subset T_{x} M^{\perp}$ que $N_{1}(x)$ pode ser um subespaço tipo tempo ou tipo espaço. Segue-se, ainda do fato de $N_{1}$ ser não degenerado, que se existe $x \in M$ tal que $N_{1}(x)$ é tipo tempo, então $N_{1}$ é tipo tempo. No caso esférico temos um comportamento análogo.

Se $N_{1}(x)$ for tipo espaço, segue-se que $\tilde{L}(x)$ também é um subespaço tipo espaço, donde temos que $\tilde{T}_{x} M^{\perp}=\tilde{L}(x) \oplus \tilde{L}^{\perp}(x)$ com $\tilde{L}^{\perp}(x)$ um subespaço tipo tempo.

É fácil verificar que o complementar ortogonal de $\tilde{L}(x)$ em $\tilde{T}_{x} M^{\perp}$ coincide com o complementar ortogonal de $N_{1}(x)$ em $T_{x} M^{\perp}$, que é paralelo na conexão $\nabla^{\perp}=\tilde{\nabla}_{\left.\right|_{T M^{\perp}}}^{\perp}$ 
de $f$. Olhando $f$ como um campo vetorial normal à imersão $\tilde{f}$, temos para cada campo $\eta$ em $\tilde{L}^{\perp}$, que

$$
X\langle f, \eta\rangle=0 \Rightarrow\left\langle\tilde{\nabla}_{X} f, \eta\right\rangle+\left\langle f, \tilde{\nabla}_{X} \eta\right\rangle=0, \quad \forall X \in \chi(M)
$$

$\operatorname{Mas}\left\langle f, \tilde{\nabla}_{X} \eta\right\rangle=0$, pois pelo que observamos acima, $\tilde{L}^{\perp}$ é paralelo. Então $\left\langle\tilde{\nabla}_{X} f, \eta\right\rangle=$ 0 implica que $\tilde{\nabla}_{X} f$ é um campo em $\tilde{L}$, pois $\tilde{L}^{\perp}$ é um subfibrado não degenerado. Se $v=n+\lambda f$ é um campo em $\tilde{L}$, onde $n$ é um campo em $N_{1}(x)$ e $\lambda: M^{n} \rightarrow \mathbb{R}$ é uma função, segue-se que

$$
\begin{aligned}
X\langle v, \eta\rangle=X\langle n+\lambda f, \eta\rangle=0, \forall X \in \chi(M) & \Rightarrow\left\langle\tilde{\nabla}_{X} n+\lambda \tilde{\nabla}_{X} f+X[\lambda] f, \eta\right\rangle=0 \\
& \Rightarrow \lambda\left\langle\tilde{\nabla}_{X} f, \eta\right\rangle=0 \\
& \Rightarrow \tilde{L} \text { é paralelo. }
\end{aligned}
$$

Se $N_{1}(x)$ for tipo tempo, teremos que $\tilde{L}$ também será um subfibrado tipo tempo, e analogamente, ao caso tipo espaço, mostra-se que $\tilde{L}$ é paralelo.

Pelo teorema (1.25) segue-se, em ambos os casos, que $\tilde{f}$ admite redução de codimensão. Explicitamente temos que

$$
\begin{aligned}
\tilde{f}(M) \subset \tilde{T}_{x} M \oplus \tilde{L}(x) & =T_{x} M \oplus N_{1}(x) \oplus \operatorname{span}\{f(x)\} \\
& =\mathbb{E}(x)
\end{aligned}
$$

ou seja, $\tilde{f}(M)$ está contido em uma $(n+q+1)$-subvariedade totalmente geodésica de $\mathbb{L}^{n+p+1}$, onde $\mathbb{E}(x)$ é isométrico a $\mathbb{R}^{n+q+1}$, se $N_{1}(x)$ for tipo espaço, ou a $\mathbb{L}^{n+q+1}$, se $N_{1}(x)$ for tipo tempo. Em ambos os casos temos que $\mathbb{E}$ é um subespaço de $\mathbb{L}^{n+p+1}$ tal que $\mathbb{E} \cap \mathbb{S}_{1}^{n+p} \neq \emptyset$. Então

$$
M^{n} \subset \mathbb{E} \cap \mathbb{S}_{1}^{n+p} \simeq\left\{\begin{array}{lll}
\mathbb{S}_{1}^{n+q}, & \text { se } N_{1} \text { for tipo tempo } \\
\mathbb{S}^{n+q}, & \text { se } N_{1} \text { for tipo espaço. }
\end{array}\right.
$$




\section{Capítulo 2}

\section{Superfícies regradas}

O objetivo deste capítulo é fornecer algumas relações geométricas que as superfícies regradas em $\mathbb{Q}^{3}(c)$ e $\mathbb{S}_{1}^{3}$ satisfazem, e que serão usadas nos próximos capítulos. $\mathrm{Na}$ seção 2.1 e 2.2 determinamos uma expressão para as curvaturas Gaussiana e média de uma superfície regrada em $\mathbb{Q}^{3}(c)$ e $\mathbb{S}_{1}^{3}$, respectivamente. Na seção 2.2 obtemos, da equação de Codazzi, um sistema que a superfície regrada em $\mathbb{Q}^{3}(c)$ deve satisfazer e na seção 2.3 falamos sobre as superfícies regradas desenvolvíveis.

\subsection{Superfícies regradas em $Q^{3}(c)$}

Seja $M^{2} \subset \mathbb{Q}^{3}(c)$ uma superfície conexa regrada imersa em $\mathbb{Q}^{3}(c), c=0, \pm 1$. De acordo com a seção (1.6), $M^{2}$ pode ser localmente parametrizada por

$$
\begin{aligned}
X(s, t) & =\exp _{e_{0}(s)}\left(t e_{1}(s)\right) \\
& =e_{0}(s) f(t)+e_{1}(s) g(t)
\end{aligned}
$$

onde as funções $f(t)$ e $g(t)$ são definidas em (1.12), $e_{0}(s)$ é uma curva em $\mathbb{Q}^{3}(c)$ e $e_{1}(s)$ é um campo unitário tangente a $\mathbb{Q}^{3}(c)$ ao longo de $e_{0}(s)$, tais que

$$
\begin{cases}\left|\dot{e}_{0}\right|=1, \quad\left\langle\dot{e}_{j}, e_{i}\right\rangle=0 \quad j, i=0,1, & \text { se } \quad c= \pm 1 \\ \left|\dot{e}_{1}\right|=1, \quad\left\langle\dot{e}_{0}, \dot{e}_{1}\right\rangle=0, & \text { se } \quad c=0 .\end{cases}
$$

Para facilitar a leitura e não deixar o texto carregado, usaremos $\langle$,$\rangle para representar$ tanto a métrica induzida em $\mathbb{S}^{3}$ como em $\mathbb{H}^{3}$. Observe que no caso $c=-1,\langle$, pode assumir valores negativos mas, por abuso de notação, também usaremos neste caso que $|\cdot|^{2}=\langle$,$\rangle .$ 
Segue-se de (2.1) que

$$
\begin{aligned}
X_{s} & =\dot{e}_{0} f(t)+\dot{e}_{1} g(t) \\
X_{t} & =e_{0} \frac{d f(t)}{d t}+e_{1} \frac{d g(t)}{d t} \\
X_{s t} & =\dot{e}_{0} \frac{d f(t)}{d t}+\dot{e}_{1} \frac{d g(t)}{d t} \\
X_{s s} & =\ddot{e}_{0} f(t)+\ddot{e}_{1} g(t) \\
X_{t t} & =-c X
\end{aligned}
$$

onde '.' é a derivação usual em relação a variável $s$ em $\mathbb{F}^{4}$ onde $\mathbb{F}^{4}=\mathbb{R}^{4}$, se $c=0,1$, ou $\mathbb{F}^{4}=\mathbb{L}^{4}$, se $c=-1$. É imediato verificar que para $c \neq 0$, temos

$$
\left\{\begin{aligned}
\left\langle X_{s}, X_{t}\right\rangle & =0 \\
\left|X_{t}\right|^{2} & =1 \\
\left|X_{s}\right|^{2} & =f^{2}(t)+g^{2}(t)\left|\dot{e}_{1}\right|^{2}+2 f(t) g(t)\left\langle\dot{e}_{0}, \dot{e}_{1}\right\rangle .
\end{aligned}\right.
$$

No caso $c=0$, tomando-se a base ortonormal $\mathcal{B}=\left\{e_{1}, \dot{e}_{1}, e_{1} \times \dot{e}_{1}\right\}$ de $\mathbb{R}^{3}$, onde $\times$ denota o produto vetorial em $\mathbb{R}^{3}$ e usando-se a notação

$$
\left\{\begin{array}{l}
F(s)=\left\langle\dot{e}_{0}, e_{1}\right\rangle \\
Q(s)=\left\langle\dot{e}_{0}, e_{1} \times \dot{e}_{1}\right\rangle \\
J(s)=\left\langle\ddot{e}_{1}, e_{1} \times \dot{e}_{1}\right\rangle \\
D(s)^{2}=Q(s)^{2}+t^{2}
\end{array}\right.
$$

segue-se por $[\mathrm{K}]$ que

$$
K=-\frac{Q^{2}}{D^{4}}, \quad H=\frac{1}{2 D^{3}}\left(J t^{2}-\dot{Q} t+Q(Q J-F)\right) .
$$

Vale observar que a curvatura Gaussiana de uma superfície regrada em $\mathbb{R}^{3}$ é sempre menor ou igual a zero.

Nos casos $c= \pm 1$, tomemos a base ortonormal $\mathcal{A}=\left\{X_{t}, \frac{X_{s}}{\left|X_{s}\right|}, X_{t} \wedge \frac{X_{s}}{\left|X_{s}\right|} \wedge X, X\right\}$ de $\mathbb{F}^{4}$, onde os três primeiros campos são tangentes a $\mathbb{Q}^{3}(c)$, sendo que os dois primeiros são tangentes a $M^{2}$, e o quarto é normal a $\mathbb{Q}^{3}(c)$.

Se $\alpha$ denota a segunda forma fundamental de $M^{2}$ e $\bar{\nabla}, \nabla$ são as conexões de Levi-Civita em $\mathbb{Q}^{3}(c)$ e $\mathbb{F}^{4}$, respectivamente, temos que

$$
\begin{aligned}
\alpha\left(X_{s}, X_{s}\right) & =\left(\bar{\nabla}_{X_{s}} X_{s}\right)^{\perp}=\nabla_{X_{s}} X_{s}-\left(\nabla_{X_{s}} X_{s}\right)^{\top}-c\left\langle\nabla_{X_{s}} X_{s}, X\right\rangle X \\
& =X_{s s}-\left\langle X_{s s}, \frac{X_{s}}{\left|X_{s}\right|}\right\rangle \frac{X_{s}}{\left|X_{s}\right|}-\left\langle X_{s s}, X_{t}\right\rangle X_{t}-c\left\langle X_{s s}, X\right\rangle X, \\
\alpha\left(X_{t}, X_{t}\right) & =X_{t t}-\left\langle X_{t t}, \frac{X_{s}}{\left|X_{s}\right|}\right\rangle \frac{X_{s}}{\left|X_{s}\right|}-\left\langle X_{t t}, X_{t}\right\rangle X_{t}-c\left\langle X_{t t}, X\right\rangle X \\
& =0,
\end{aligned}
$$




$$
\alpha\left(X_{s}, X_{t}\right)=X_{s t}-\left\langle X_{s t}, \frac{X_{s}}{\left|X_{s}\right|}\right\rangle \frac{X_{s}}{\left|X_{s}\right|}-\left\langle X_{s t}, X_{t}\right\rangle X_{t}-c\left\langle X_{s t}, X\right\rangle X
$$

donde após cálculos simples segue-se que

$$
\left|\alpha\left(X_{s}, X_{s}\right)\right|^{2}=\left|X_{s s}\right|^{2}-\frac{\left\langle X_{s s}, X_{s}\right\rangle^{2}}{\left|X_{s}\right|^{2}}-\left\langle X_{s s}, X_{t}\right\rangle^{2}-c\left\langle X_{s s}, X\right\rangle^{2}
$$

e

$$
\left|\alpha\left(X_{s}, X_{t}\right)\right|^{2}=\left|X_{s t}\right|^{2}-\frac{\left\langle X_{s t}, X_{s}\right\rangle^{2}}{\left|X_{s}\right|^{2}} .
$$

Segue-se pelas igualdades anteriores que o vetor curvatura média de uma superfície regrada em $\mathbb{Q}^{3}(c)$ é dado por

$$
\vec{H}(s, t)=\frac{1}{2\left|X_{s}\right|^{2}} \alpha\left(X_{s}, X_{s}\right)
$$

e portanto, a função curvatura média da superfície regrada é dada por

$$
H=|\vec{H}(s, t)|=\frac{1}{2\left|X_{s}\right|^{2}}\left|\alpha\left(X_{s}, X_{s}\right)\right|
$$

Além disso, denotando por $K$ e $\bar{K}$ as curvaturas seccionais de $M^{2}$ e $\mathbb{Q}^{3}(c)$, respectivamente, segue-se por Gauss que

$$
\begin{aligned}
K\left(\frac{X_{s}}{\left|X_{s}\right|}, X_{t}\right) & =\bar{K}\left(\frac{X_{s}}{\left|X_{s}\right|}, X_{t}\right)+\left\langle\alpha\left(\frac{X_{s}}{\left|X_{s}\right|}, \frac{X_{s}}{\left|X_{s}\right|}\right), \alpha\left(X_{t}, X_{t}\right)\right\rangle+ \\
& -\left|\alpha\left(\frac{X_{s}}{\left|X_{s}\right|}, X_{t}\right)\right|^{2} \\
& =c-\frac{\left|\alpha\left(X_{s}, X_{t}\right)\right|^{2}}{\left|X_{s}\right|^{2}}
\end{aligned}
$$

O nosso próximo passo é escrever as funções curvatura Gaussiana e média em função da base $\mathcal{A}$. Observando-se que

$$
\dot{f}(t)=-c g(t) \quad \text { e } \quad \dot{g}(t)=f(t)
$$

é fácil verificar que

$$
\begin{aligned}
& X_{t} \wedge X_{s} \wedge X=\left(\frac{d g(t)}{d t} f(t)-\frac{d f(t)}{d t} g(t)\right) e_{1} \wedge X_{s} \wedge e_{0} \\
& =\left(\frac{d g(t)}{d t} f^{2}(t)-g(t) f(t) \frac{d f(t)}{d t}\right) e_{1} \wedge \dot{e}_{0} \wedge e_{0}+ \\
& +\left(g(t) f(t) \frac{d g(t)}{d t}-g^{2}(t) \frac{d f(t)}{d t}\right) e_{1} \wedge \dot{e}_{1} \wedge e_{0} \\
& =f(t) e_{1} \wedge \dot{e}_{0} \wedge e_{0}+g(t) e_{1} \wedge \dot{e}_{1} \wedge e_{0},
\end{aligned}
$$


donde segue-se que

$$
\begin{aligned}
\left\langle X_{s t}, X_{t} \wedge X_{s} \wedge X\right\rangle & =-c g^{2}(t)\left\langle\dot{e}_{0}, e_{1} \wedge \dot{e}_{1} \wedge e_{0}\right\rangle+f^{2}(t)\left\langle\dot{e}_{1}, e_{1} \wedge \dot{e}_{0} \wedge e_{0}\right\rangle \\
& =-\left\langle\dot{e}_{0}, e_{1} \wedge \dot{e}_{1} \wedge e_{0}\right\rangle
\end{aligned}
$$

$\mathrm{e}$

$$
\begin{aligned}
\left\langle X_{s s}, X_{t} \wedge X_{s} \wedge X\right\rangle & =f^{2}(t)\left\langle\ddot{e}_{0}, e_{1} \wedge \dot{e}_{0} \wedge e_{0}\right\rangle+f(t) g(t)\left\langle\ddot{e}_{0}, e_{1} \wedge \dot{e}_{1} \wedge e_{0}\right\rangle+ \\
& +f(t) g(t)\left\langle\ddot{e}_{1}, e_{1} \wedge \dot{e}_{0} \wedge e_{0}\right\rangle+g^{2}(t)\left\langle\ddot{e}_{1}, e_{1} \wedge \dot{e}_{1} \wedge e_{0}\right\rangle
\end{aligned}
$$

Como $X_{s t}$ e $X_{s s}$ pertencem a $\mathbb{F}^{4}$ temos, usando a base $\mathcal{A}$, que

$$
X_{s t}=\left\langle X_{s t}, X_{s}\right\rangle \frac{X_{s}}{\left|X_{s}\right|^{2}}+\left\langle X_{s t}, X_{t} \wedge X_{s} \wedge X\right\rangle X_{t} \wedge \frac{X_{s}}{\left|X_{s}\right|^{2}} \wedge X
$$

e

$$
\begin{aligned}
X_{s s} & =\left\langle X_{s s}, X_{t}\right\rangle X_{t}+\left\langle X_{s s}, X_{s}\right\rangle \frac{X_{s}}{\left|X_{s}\right|^{2}}+ \\
& +\left\langle X_{s s}, X_{t} \wedge X_{s} \wedge X\right\rangle X_{t} \wedge \frac{X_{s}}{\left|X_{s}\right|^{2}} \wedge X+c\left\langle X_{s s}, X\right\rangle X
\end{aligned}
$$

donde segue-se que

$$
\left|X_{s t}\right|^{2}=\frac{\left\langle X_{s t}, X_{s}\right\rangle^{2}}{\left|X_{s}\right|^{2}}+\frac{\left\langle X_{s t}, X_{t} \wedge X_{s} \wedge X\right\rangle^{2}}{\left|X_{s}\right|^{2}}
$$

e

$$
\left|X_{s s}\right|^{2}=\left\langle X_{s s}, X_{t}\right\rangle^{2}+\frac{\left\langle X_{s s}, X_{s}\right\rangle^{2}}{\left|X_{s}\right|^{2}}+\frac{\left\langle X_{s s}, X_{t} \wedge X_{s} \wedge X\right\rangle^{2}}{\left|X_{s}\right|^{2}}+c\left\langle X_{s s}, X\right\rangle^{2}
$$

Logo por (2.5), (2.6), (2.7) e (2.8) segue-se que

$$
\begin{aligned}
H & =\frac{1}{2\left|X_{s}\right|^{3}}\left\langle X_{s s}, X_{t} \wedge X_{s} \wedge X\right\rangle \\
K & =c-\frac{\left\langle X_{s t}, X_{t} \wedge X_{s} \wedge X\right\rangle^{2}}{\left|X_{s}\right|^{4}}
\end{aligned}
$$

Usando-se a notação

$$
\left\{\begin{aligned}
F(s)= & \left\langle\ddot{e}_{0}, e_{1} \wedge \dot{e}_{0} \wedge e_{0}\right\rangle, \\
D(s)= & \left\langle\ddot{e}_{1}, e_{1} \wedge \dot{e}_{1} \wedge e_{0}\right\rangle, \\
G(s)=\left\langle\ddot{e}_{1}, e_{1} \wedge \dot{e}_{0} \wedge e_{0}\right\rangle, & \\
L(s)=\left\langle\ddot{e}_{0}, e_{1} \wedge \dot{e}_{1} \wedge e_{0}\right\rangle, & \\
Q(s)= & \left\{\begin{array}{lll}
\left\langle\dot{e}_{0}, e_{1} \wedge \dot{e}_{1} \wedge e_{0}\right\rangle, & \text { se } c=1, \\
\left\langle\dot{e}_{1}, e_{1} \wedge \dot{e}_{0} \wedge e_{0}\right\rangle, & \text { se } & c=-1,
\end{array}\right.
\end{aligned}\right.
$$


segue-se por (2.9) que as curvaturas Gaussiana e média de uma superfície regrada em $\mathbb{Q}^{3}(c)$ são dadas por

$$
\begin{cases}K=1-\frac{Q^{2}}{\left|X_{s}\right|^{4}}, & H=\frac{h}{2\left|X_{s}\right|^{3}}, \text { se } c=1, \\ K=-1-\frac{Q^{2}}{\left|X_{s}\right|^{4}}, & H=\frac{h}{2\left|X_{s}\right|^{3}}, \text { se } c=-1,\end{cases}
$$

onde

$$
h=h(s, t)=f^{2}(t) F(s)+g^{2}(t) D(s)+(L(s)+G(s)) f(t) g(t) .
$$

É imediato por (2.11) a generalização de um resultado de $\mathbb{R}^{3}$, isto é, a curvatura Gaussiana de uma superfície regrada em $\mathbb{Q}^{3}(c)$, é sempre menor ou igual a $c$.

Segue-se pela expressão de $Q(s)$ que

$$
\begin{aligned}
\dot{Q} & =c\left(\left\langle\ddot{e}_{0}, e_{1} \wedge \dot{e}_{1} \wedge e_{0}\right\rangle+\left\langle\dot{e}_{0}, e_{1} \wedge \ddot{e}_{1} \wedge e_{0}\right\rangle\right) \\
& =c(L-G)
\end{aligned}
$$

e

$$
\begin{aligned}
Q^{2} & =\left(\operatorname{det}\left[\begin{array}{c}
\dot{e}_{0} \\
e_{1} \\
\dot{e}_{1} \\
e_{0}
\end{array}\right]\right)^{2}=\operatorname{det}\left(\left[\begin{array}{cccc}
1 & 0 & \left\langle\dot{e}_{1}, \dot{e}_{0}\right\rangle & 0 \\
0 & 1 & 0 & 0 \\
\left\langle\dot{e}_{0}, \dot{e}_{1}\right\rangle & 0 & \left|\dot{e}_{1}\right|^{2} & 0 \\
0 & 0 & 0 & 1
\end{array}\right]\right) \\
& =\left|\dot{e}_{1}\right|^{2}-\left\langle\dot{e}_{0}, \dot{e}_{1}\right\rangle^{2} .
\end{aligned}
$$

\subsection{Superfícies regradas tipo espaço em $\mathbb{S}_{1}^{3}$}

Seja $M^{2} \subset \mathbb{S}_{1}^{3}$ uma superfície conexa, tipo espaço, regrada imersa em $\mathbb{S}_{1}^{3}$ que pode ser, de acordo com a seção (1.7), parametrizada localmente por

$$
X(s, t)=e_{0}(s) \cos t+e_{1}(s) \sin t
$$

onde $e_{0}$ é uma curva tipo espaço em $M^{2}$ e $e_{1}$ é um campo tangente à $M^{2}$ ao longo de $e_{0}\left(\left\langle e_{0}, e_{1}\right\rangle=0\right)$, tal que $\left\langle e_{1}, e_{1}\right\rangle=1$. Além disso, suponhamos que $\left\langle\dot{e}_{0}, \dot{e}_{1}\right\rangle=0 \mathrm{e}$ $\left\langle\dot{e}_{1}, \dot{e}_{1}\right\rangle=1$, onde '!' é a derivação usual em relação a variável $s \mathrm{em} \mathbb{L}^{4}$. 
Por derivação direta, segue-se de (2.13) que

$$
\begin{aligned}
& X_{s}=\dot{e}_{0} \cos t+\dot{e}_{1} \sin t \\
& X_{t}=-e_{0} \sin t+e_{1} \cos t \\
& X_{s t}=-\dot{e}_{0} \sin t+\dot{e}_{1} \cos t \\
& X_{s s}=\ddot{e}_{0} \cos t+\ddot{e}_{1} \sin t \\
& X_{t t}=-X,
\end{aligned}
$$

donde temos que

$$
\left\{\begin{array}{l}
\left\langle X_{s}, X_{t}\right\rangle=\left\langle\dot{e}_{0}, e_{1}\right\rangle \\
\left\langle X_{t}, X_{t}\right\rangle=1 \\
\left\langle X_{s}, X_{s}\right\rangle=\left\langle\dot{e}_{0}, \dot{e}_{0}\right\rangle \cos ^{2} t+\sin ^{2} t
\end{array}\right.
$$

Note que $\left\langle X_{s}, X_{s}\right\rangle>0$.

Consideremos a base ortonormal $\mathcal{D}=\left\{X_{t}, \frac{\tilde{X}_{s}}{\left|\tilde{X}_{s}\right|}, X_{t} \wedge \frac{\tilde{X}_{s}}{\left|\tilde{X}_{s}\right|} \wedge X, X\right\}$ de $\mathbb{L}^{4}$, onde $\tilde{X}_{s}=X_{s}-\left\langle X_{t}, X_{s}\right\rangle X_{t}$. Observe que os dois primeiros campos de $\mathcal{D}$ são tangentes a $M^{2}$, isto é, determinam em cada ponto um vetor tipo espaço e o terceiro campo é tangente a $\mathbb{S}_{1}^{3}$ e normal a $M^{2}$, isto é, em cada ponto determina um vetor tipo tempo.

Se $\alpha$ denota a segunda forma fundamental de $M^{2}$ segue-se, analogamente à seção anterior, que

$$
\begin{gathered}
\alpha\left(\tilde{X}_{s}, \tilde{X}_{s}\right)=\alpha\left(X_{s}, X_{s}\right)-2\left\langle X_{t}, X_{s}\right\rangle \alpha\left(X_{s}, X_{t}\right)+\left\langle X_{t}, X_{s}\right\rangle^{2} \alpha\left(X_{t}, X_{t}\right), \\
\alpha\left(\tilde{X}_{s}, X_{t}\right)=\alpha\left(X_{s}, X_{t}\right)-\left\langle X_{t}, X_{s}\right\rangle \alpha\left(X_{t}, X_{t}\right)
\end{gathered}
$$

onde

$$
\begin{gathered}
\alpha\left(X_{s}, X_{s}\right)=X_{s s}-\left\langle X_{s s}, \tilde{X}_{s}\right\rangle \frac{\tilde{X}_{s}}{\left|\tilde{X}_{s}\right|^{2}}-\left\langle X_{s s}, X_{t}\right\rangle X_{t}-\left\langle X_{s s}, X\right\rangle X \\
\alpha\left(X_{t}, X_{t}\right)=X_{t t}-\left\langle X_{t t}, \tilde{X}_{s}\right\rangle \frac{\tilde{X}_{s}}{\left|\tilde{X}_{s}\right|^{2}}-\left\langle X_{t t}, X_{t}\right\rangle X_{t}-\left\langle X_{t t}, X\right\rangle X
\end{gathered}
$$

$\mathrm{e}$

$$
\alpha\left(X_{s}, X_{t}\right)=X_{s t}-\left\langle X_{s t}, \tilde{X}_{s}\right\rangle \frac{\tilde{X}_{s}}{\left|\tilde{X}_{s}\right|^{2}}-\left\langle X_{s t}, X_{t}\right\rangle X_{t}-\left\langle X_{s t}, X\right\rangle X
$$

donde temos que o vetor curvatura média de uma superfície regrada tipo espaço em $\mathbb{S}_{1}^{3}$ é dado por

$$
\vec{H}(s, t)=\frac{1}{2\left|\tilde{X}_{s}\right|^{2}} \alpha\left(\tilde{X}_{s}, \tilde{X}_{s}\right)
$$


e portanto, a função curvatura média da superfície regrada é dada por

$$
\mathcal{H}=\langle\vec{H}, \vec{H}\rangle=\frac{1}{4\left|\tilde{X}_{s}\right|^{4}}\left\langle\alpha\left(\tilde{X}_{s}, \tilde{X}_{s}\right), \alpha\left(\tilde{X}_{s}, \tilde{X}_{s}\right)\right\rangle .
$$

Observe que $\mathcal{H}$ é um número negativo. Denotando por $K$ e $\bar{K}$ as curvaturas seccionais de $M^{2}$ e $\mathbb{S}_{1}^{3}$, respectivamente, segue-se por Gauss que

$$
K\left(\frac{\tilde{X}_{s}}{\left|\tilde{X}_{s}\right|}, X_{t}\right)=1-\frac{\left\langle\alpha\left(X_{s}, X_{t}\right), \alpha\left(X_{s}, X_{t}\right)\right\rangle}{\left|\tilde{X}_{s}\right|^{2}}
$$

Passemos agora a escrever (2.14) e (2.15) em função da base $\mathcal{D}$. Seguindo cálculos análogos à seção anterior, temos que

$$
\begin{aligned}
X_{t} \wedge \tilde{X}_{s} \wedge X & =X_{t} \wedge X_{s} \wedge X \\
& =\cos t e_{1} \wedge \dot{e}_{0} \wedge e_{0}+\sin t e_{1} \wedge \dot{e}_{1} \wedge e_{0}
\end{aligned}
$$

donde segue-se que

$$
\left\langle X_{s t}, X_{t} \wedge X_{s} \wedge X\right\rangle=-\left\langle\dot{e}_{0}, e_{1} \wedge \dot{e}_{1} \wedge e_{0}\right\rangle
$$

$\mathrm{e}$

$$
\begin{aligned}
\left\langle X_{s s}, X_{t} \wedge X_{s} \wedge X\right\rangle & =\cos ^{2} t\left\langle\ddot{e}_{0}, e_{1} \wedge \dot{e}_{0} \wedge e_{0}\right\rangle+\cos t \sin t\left(\left\langle\ddot{e}_{0}, e_{1} \wedge \dot{e}_{1} \wedge e_{0}\right\rangle+\right. \\
& \left.+\left\langle\ddot{e}_{1}, e_{1} \wedge \dot{e}_{0} \wedge e_{0}\right\rangle\right)+\sin ^{2} t\left\langle\ddot{e}_{1}, e_{1} \wedge \dot{e}_{1} \wedge e_{0}\right\rangle .
\end{aligned}
$$

Além disso, como $X_{s t}$ e $X_{s s}$ pertencem a $\mathbb{L}^{4}$, temos usando-se a base $\mathcal{D}$ que

$$
\begin{gathered}
X_{s t}=\left\langle X_{s t}, \tilde{X}_{s}\right\rangle \frac{\tilde{X}_{s}}{\left|\tilde{X}_{s}\right|^{2}}-\left\langle X_{s t}, X_{t} \wedge X_{s} \wedge X\right\rangle X_{t} \wedge \frac{X_{s}}{\left|\tilde{X}_{s}\right|^{2}} \wedge X+ \\
+\left\langle X_{s t}, X\right\rangle X
\end{gathered}
$$

$\mathrm{e}$

$$
\begin{aligned}
X_{s s}= & \left\langle X_{s s}, X_{t}\right\rangle X_{t}+\left\langle X_{s s}, \tilde{X}_{s}\right\rangle \frac{\tilde{X}_{s}}{\left|\tilde{X}_{s}\right|^{2}}+ \\
& -\left\langle X_{s s}, X_{t} \wedge X_{s} \wedge X\right\rangle X_{t} \wedge \frac{X_{s}}{\left|\tilde{X}_{s}\right|^{2}} \wedge X+\left\langle X_{s s}, X\right\rangle X
\end{aligned}
$$

donde segue-se que

$$
\left|X_{s t}\right|^{2}=\frac{\left\langle X_{s t}, \tilde{X}_{s}\right\rangle^{2}}{\left|\tilde{X}_{s}\right|^{2}}-\frac{\left\langle X_{s t}, X_{t} \wedge X_{s} \wedge X\right\rangle^{2}}{\left|\tilde{X}_{s}\right|^{2}}+\left\langle X_{s t}, X\right\rangle^{2}
$$




$$
\left|X_{s s}\right|^{2}=\left\langle X_{s s}, X_{t}\right\rangle^{2}+\frac{\left\langle X_{s s}, \tilde{X}_{s}\right\rangle^{2}}{\left|\tilde{X}_{s}\right|^{2}}-\frac{\left\langle X_{s s}, X_{t} \wedge X_{s} \wedge X\right\rangle^{2}}{\left|\tilde{X}_{s}\right|^{2}}+\left\langle X_{s s}, X\right\rangle^{2}
$$

$$
\begin{aligned}
\left\langle X_{s t}, X_{s s}\right\rangle= & \frac{\left\langle X_{s t}, \tilde{X}_{s}\right\rangle\left\langle X_{s s}, \tilde{X}_{s}\right\rangle}{\left|\tilde{X}_{s}\right|^{2}}+\left\langle X_{s t}, X\right\rangle\left\langle X_{s s}, X\right\rangle+ \\
& -\frac{\left\langle X_{s t}, X_{t} \wedge X_{s} \wedge X\right\rangle\left\langle X_{s s}, X_{t} \wedge X_{s} \wedge X\right\rangle}{\left|\tilde{X}_{s}\right|^{2}}
\end{aligned}
$$

Após cálculos elementares mostra-se que

$$
\begin{aligned}
\left|\alpha\left(\tilde{X}_{s}, \tilde{X}_{s}\right)\right|^{2}= & \left|\alpha\left(X_{s}, X_{s}\right)\right|^{2}+4\left\langle X_{t}, X_{s}\right\rangle\left|\alpha\left(X_{s}, X_{t}\right)\right|^{2}+ \\
& -4\left\langle X_{t}, X_{s}\right\rangle\left\langle\alpha\left(X_{s}, X_{t}\right), \alpha\left(X_{s}, X_{s}\right)\right\rangle, \\
\left|\alpha\left(X_{s}, X_{s}\right)\right|^{2}= & \left|X_{s s}\right|^{2}-\left\langle X_{s s}, X\right\rangle^{2}-\left\langle X_{s s}, X_{t}\right\rangle^{2}+ \\
& -\frac{1}{\left|\tilde{X}_{s}\right|^{2}}\left(\left\langle X_{s s}, X_{s}\right\rangle-\left\langle X_{t}, X_{s}\right\rangle\left\langle X_{s s}, X_{t}\right\rangle\right)^{2}, \\
\left|\alpha\left(X_{t}, X_{s}\right)\right|^{2}=\mid & \left|X_{s t}\right|^{2}-\left\langle X_{s t}, X\right\rangle^{2}+ \\
& -\frac{1}{\left|\tilde{X}_{s}\right|^{2}}\left(\left\langle X_{s t}, X_{s}\right\rangle-\left\langle X_{t}, X_{s}\right\rangle\left\langle X_{s t}, X_{t}\right\rangle\right)^{2}, \\
\left|\alpha\left(X_{t}, \tilde{X}_{s}\right)\right|^{2}=\left|\alpha\left(X_{t}, X_{s}\right)\right|^{2}, & \mid\left\langle X_{s s}, X_{s t}\right\rangle-\left\langle X_{s t}, X\right\rangle\left\langle X_{s s}, X\right\rangle+ \\
\left\langle\alpha\left(X_{s}, X_{s}\right), \alpha\left(X_{s}, X_{t}\right)\right\rangle= & -\frac{1}{\left|\tilde{X}_{s}\right|^{2}}\left(\left\langle X_{s t}, X_{s}\right\rangle\right) \\
& \left(\left\langle X_{s s}, X_{s}\right\rangle-\left\langle X_{t}, X_{s}\right\rangle\left\langle X_{s s}, X_{t}\right\rangle\right) .
\end{aligned}
$$

Logo pelas igualdades anteriores, temos que (2.14) e (2.15) se reescrevem como

$$
\begin{aligned}
\mathcal{H}= & \frac{1}{4\left|\tilde{X}_{s}\right|^{4}}\left(-\frac{\left\langle X_{s s}, X_{t} \wedge X_{s} \wedge X\right\rangle^{2}}{\left|\tilde{X}_{s}\right|^{2}}-4\left\langle X_{t}, X_{s}\right\rangle^{2} \frac{\left\langle X_{s s}, X_{t} \wedge X_{s} \wedge X\right\rangle^{2}}{\left|\tilde{X}_{s}\right|^{2}}+\right. \\
& \left.\quad+4 \frac{\left\langle X_{s}, X_{t}\right\rangle\left\langle X_{s s}, X_{t} \wedge X_{s} \wedge X\right\rangle\left\langle X_{s t}, X_{t} \wedge X_{s} \wedge X\right\rangle}{\left|\tilde{X}_{s}\right|^{2}}\right) \\
= & -\frac{1}{4\left|\tilde{X}_{s}\right|^{6}}\left(\left\langle X_{s s}, X_{t} \wedge X_{s} \wedge X\right\rangle+\right. \\
& \left.\quad-2\left\langle X_{s}, X_{t}\right\rangle\left\langle X_{s t}, X_{t} \wedge X_{s} \wedge X\right\rangle\right)^{2} \\
K= & 1+\frac{1}{\left|\tilde{X}_{s}\right|^{4}}\left\langle X_{s t}, X_{t} \wedge X_{s} \wedge X\right\rangle^{2}
\end{aligned}
$$

Usando-se a notação

$$
\left\{\begin{array}{l}
A(s)=\left|\dot{e}_{0}\right|^{2}-\left\langle\dot{e}_{0}, e_{1}\right\rangle^{2} \\
B(s)=1-\left\langle\dot{e}_{0}, e_{1}\right\rangle^{2} \\
P(s, t)=A(s) \cos ^{2} t+B(s) \sin ^{2} t \\
Q(s, t)=\left\langle\dot{e}_{1}, \dot{e}_{0} \wedge e_{1} \wedge e_{0}\right\rangle \\
h(s, t)=F(s) \cos ^{2} t+D(s) \sin ^{2} t+(L(s)+G(s)) \cos t \sin t
\end{array}\right.
$$


onde $F(s), D(s), L(s)$ e $G(s)$ são dados em (2.10), mostramos que as curvaturas Gaussiana e média de uma superfície tipo espaço em $\mathbb{S}_{1}^{3}$ são dadas por

$$
K=1+\frac{Q^{2}}{P^{2}}, \quad \mathcal{H}=-\frac{\left(h-2 Q\left\langle\dot{e}_{0}, \dot{e}_{1}\right\rangle\right)^{2}}{4 P^{3}} .
$$

Observe que $Q^{2}=A B$. De fato,

$$
\begin{aligned}
Q^{2} & =\left(\operatorname{det}\left[\begin{array}{c}
\dot{e}_{1} \\
\dot{e}_{0} \\
e_{1} \\
e_{0}
\end{array}\right]\right)^{2}= \\
& =\operatorname{det}\left(\left[\begin{array}{cccc}
1 & 0 & 0 & \left\langle\dot{e}_{1}, e_{0}\right\rangle \\
0 & \left|\dot{e}_{0}\right|^{2} & \left\langle\dot{e}_{0}, e_{1}\right\rangle & 0 \\
0 & \left\langle\dot{e}_{0}, e_{1}\right\rangle & 1 & 0 \\
\left\langle\dot{e}_{1}, e_{0}\right\rangle & 0 & 0 & 1
\end{array}\right]\right) \\
& =\left(\left|\dot{e}_{0}\right|^{2}-\left\langle\dot{e}_{0}, e_{1}\right\rangle^{2}\right) \\
& =A B
\end{aligned}
$$

\subsection{Equação de Codazzi para superfícies em $\mathbb{S}^{3} \mathrm{e}$ $\mathbf{H}^{3}$}

Será importante em cálculos futuros, determinarmos a equação de Codazzi de uma superfície regrada em $\mathbb{Q}^{3}(c)$ em termos dos campos coordenados da parametrização dada em (2.1). Segue-se de (1.1) que a equação de Codazzi de uma superfície parametrizada como em (2.1) é dada por:

$$
\left(\tilde{\nabla}_{X_{s}} A_{\xi}\right) X_{t}=\left(\tilde{\nabla}_{X_{t}} A_{\xi}\right) X_{s}
$$

onde $\left(\tilde{\nabla}_{X} A_{\xi}\right) Y=\tilde{\nabla}_{X}\left(A_{\xi} Y\right)-A_{\xi} \tilde{\nabla}_{X} Y$, com $\tilde{\nabla}$ a conexão de Levi-Civita da superfície e $X_{s}, X_{t}$ e $\xi=X_{t} \wedge \frac{X_{s}}{\left|X_{s}\right|} \wedge X$ definidos como anteriormente.

Os coeficientes da segunda forma fundamental da imersão $X$ são dados por

$$
\left\{\begin{array}{l}
e=\left\langle\alpha\left(X_{t}, X_{t}\right), \xi\right\rangle \\
f=\left\langle\alpha\left(X_{t}, X_{s}\right), \xi\right\rangle \\
g=\left\langle\alpha\left(X_{s}, X_{s}\right), \xi\right\rangle
\end{array}\right.
$$

ou equivalentemente,

$$
e=0, \quad f=-c \frac{Q}{\left|X_{s}\right|}, \quad g=\frac{h}{\left|X_{s}\right|} .
$$


Com a notação anterior temos que

$$
\begin{aligned}
\left(\tilde{\nabla}_{X_{t}} A_{\xi}\right) X_{s}= & \tilde{\nabla}_{X_{t}}\left(\left\langle A_{\xi} X_{s}, X_{t}\right\rangle X_{t}+\left\langle A_{\xi} X_{s}, X_{s}\right\rangle \frac{X_{s}}{\left|X_{s}\right|^{2}}\right)+ \\
& -\left\langle A_{\xi} \tilde{\nabla}_{X_{t}} X_{s}, X_{t}\right\rangle X_{t}-\left\langle A_{\xi} \tilde{\nabla}_{X_{t}} X_{s}, X_{s}\right\rangle \frac{X_{s}}{\left|X_{s}\right|^{2}} \\
= & \tilde{\nabla}_{X_{t}}\left(f X_{t}+g \frac{X_{s}}{\left|X_{s}\right|^{2}}\right)-\left\langle\alpha\left(\tilde{\nabla}_{X_{t}} X_{s}, X_{t}\right), \xi\right\rangle X_{t}+ \\
& -\left\langle\alpha\left(\tilde{\nabla}_{X_{t}} X_{s}, X_{s}\right), \xi\right\rangle \frac{X_{s}}{\left|X_{s}\right|^{2}}
\end{aligned}
$$

e analogamente mostra-se que

$$
\begin{aligned}
\left(\tilde{\nabla}_{X_{s}} A_{\xi}\right) X_{t}=\tilde{\nabla}_{X_{s}} & \left(f \frac{X_{s}}{\left|X_{s}\right|^{2}}\right)-\left\langle\alpha\left(\tilde{\nabla}_{X_{s}} X_{t}, X_{t}\right), \xi\right\rangle X_{t}+ \\
& -\left\langle\alpha\left(\tilde{\nabla}_{X_{s}} X_{t}, X_{s}\right), \xi\right\rangle \frac{X_{s}}{\left|X_{s}\right|^{2}} .
\end{aligned}
$$

Então a equação de Codazzi de uma superfície regrada em $\mathbb{Q}^{3}(c)$ se resume a

$$
\tilde{\nabla}_{X_{t}}\left(f X_{t}+g \frac{X_{s}}{\left|X_{s}\right|^{2}}\right)=\tilde{\nabla}_{X_{s}}\left(f \frac{X_{s}}{\left|X_{s}\right|^{2}}\right) \text {. }
$$

Desenvolvendo-se a igualdade anterior temos que

$$
f_{t} X_{t}+g_{t} \frac{X_{s}}{\left|X_{s}\right|^{2}}+f \tilde{\nabla}_{X_{t}} X_{t}+g \tilde{\nabla}_{X_{t}} \frac{X_{s}}{\left|X_{s}\right|^{2}}=f_{s} \frac{X_{s}}{\left|X_{s}\right|^{2}}+f \tilde{\nabla}_{X_{s}} \frac{X_{s}}{\left|X_{s}\right|^{2}}
$$

ou equivalentemente,

$$
\left\{\begin{array}{l}
f_{t}+f\left\langle\tilde{\nabla}_{X_{t}} X_{t}, X_{t}\right\rangle+g\left\langle\tilde{\nabla}_{X_{t}} \frac{X_{s}}{\left|X_{s}\right|^{2}}, X_{t}\right\rangle=f\left\langle\tilde{\nabla}_{X_{s}} \frac{X_{s}}{\left|X_{s}\right|^{2}}, X_{t}\right\rangle \\
g_{t}+f\left\langle\tilde{\nabla}_{X_{t}} X_{t}, X_{s}\right\rangle+g\left\langle\tilde{\nabla}_{X_{t}} \frac{X_{s}}{\left|X_{s}\right|^{2}}, X_{s}\right\rangle=f_{s}+f\left\langle\tilde{\nabla}_{X_{s}} \frac{X_{s}}{\left|X_{s}\right|^{2}}, X_{s}\right\rangle .
\end{array}\right.
$$

Observando-se que

$$
\begin{gathered}
\tilde{\nabla}_{X_{s}} \frac{X_{s}}{\left|X_{s}\right|^{2}}=-2 \frac{\left\langle X_{s s}, X_{s}\right\rangle}{\left|X_{s}\right|^{4}} X_{s}+\frac{1}{\left|X_{s}\right|^{2}}\left(X_{s s}-\left\langle X_{s s}, X\right\rangle X+\right. \\
\left.-\left\langle X_{s s}, X_{t} \wedge X_{s} \wedge X\right\rangle X_{t} \wedge \frac{X_{s}}{\left|X_{s}\right|^{2}} \wedge X\right)
\end{gathered}
$$

$\mathrm{e}$

$$
\begin{gathered}
\tilde{\nabla}_{X_{t}} \frac{X_{s}}{\left|X_{s}\right|^{2}}=-2 \frac{\left\langle X_{s t}, X_{s}\right\rangle}{\left|X_{s}\right|^{4}} X_{s}+\frac{1}{\left|X_{s}\right|^{2}}\left(X_{s t}-\left\langle X_{s t}, X\right\rangle X+\right. \\
\left.-\left\langle X_{s s}, X_{t} \wedge X_{s} \wedge X\right\rangle X_{t} \wedge \frac{X_{s}}{\left|X_{s}\right|^{2}} \wedge X\right)
\end{gathered}
$$


temos, após algumas simplificações, que

$$
\begin{aligned}
f_{t} & =-f \frac{\left\langle X_{s}, X_{s t}\right\rangle}{\left|X_{s}\right|^{2}} \\
g_{t}-f_{s} & =-f \frac{\left\langle X_{s}, X_{s s}\right\rangle}{\left|X_{s}\right|^{2}}+g \frac{\left\langle X_{s}, X_{s t}\right\rangle}{\left|X_{s}\right|^{2}}
\end{aligned}
$$

Agora segue-se por derivação direta de (2.20) que

$$
f_{t}=c \frac{Q\left(\left|X_{s}\right|\right)_{t}}{\left|X_{s}\right|^{2}}=c \frac{Q\left\langle X_{s}, X_{s t}\right\rangle}{\left|X_{s}\right|^{3}}
$$

ou seja, apenas reobtemos (2.21). Ainda por (2.20) temos que

$$
f_{s}=\frac{c\left(Q\left\langle X_{s}, X_{s s}\right\rangle-\dot{Q}\left|X_{s}\right|^{2}\right)}{\left|X_{s}\right|^{3}}
$$

$\mathrm{e}$

$$
g_{t}=\frac{h_{t}\left|X_{s}\right|^{2}-h\left\langle X_{s}, X_{s t}\right\rangle}{\left|X_{s}\right|^{3}},
$$

donde a igualdade (2.22) pode ser reescrita como

$$
h_{t}\left|X_{s}\right|^{2}-2 h\left\langle X_{s}, X_{s t}\right\rangle-c 2 Q\left\langle X_{s}, X_{s s}\right\rangle+c \dot{Q}\left|X_{s}\right|^{2}=0,
$$

ou equivalentemente,

$$
c\left(\frac{Q}{\left|X_{s}\right|^{2}}\right)_{s}+\left(\frac{h}{\left|X_{s}\right|^{2}}\right)_{t}=0 .
$$

Logo se $M^{2} \subset \mathbb{Q}^{3}(c)$ é uma superfície regrada, então $M^{2}$ deve satisfazer a relação dada em (2.24).

Usando-se (2.10) podemos ainda reescrever (2.23) como

$$
\begin{aligned}
f^{2}(t) & \left(2 L-2 F\left\langle\dot{e}_{0}, \dot{e}_{1}\right\rangle\right)+ \\
& g^{2}(t)\left(-2\left|\dot{e}_{1}\right|^{2} G+2 D\left\langle\dot{e}_{0}, \dot{e}_{1}\right\rangle-c Q\left|\dot{e}_{1}\right|^{2}\right)+ \\
& f(t) g(t)\left(2 D-2 F\left|\dot{e}_{1}\right|^{2}-2(G-L)\left\langle\dot{e}_{0}, \dot{e}_{1}\right\rangle-2 c Q\left\langle\dot{e}_{0}, \dot{e}_{1}\right\rangle\right)=0,
\end{aligned}
$$

donde segue-se que se $M^{2} \subset \mathbb{Q}^{3}(c)$ é uma superfície regrada, então $M^{2}$ deve verificar

$$
\left\{\begin{array}{l}
L-F\left\langle\dot{e}_{0}, \dot{e}_{1}\right\rangle=0 \\
-2\left|\dot{e}_{1}\right|^{2} G+2 D\left\langle\dot{e}_{0}, \dot{e}_{1}\right\rangle-c Q\left|\dot{e}_{1}\right|^{2}=0 \\
D-F\left|\dot{e}_{1}\right|^{2}-(G-L)\left\langle\dot{e}_{0}, \dot{e}_{1}\right\rangle-c Q\left\langle\dot{e}_{0}, \dot{e}_{1}\right\rangle=0
\end{array}\right.
$$

pois $f^{2}(t), g^{2}(t)$ e $f(t) g(t)$ são linearmente independentes como funções de $t$. 


\subsection{Superfícies regradas com curvatura Gaussiana constante em $\mathbb{Q}^{3}(c)$ e $\mathbb{S}_{1}^{3}$}

Existem vários trabalhos em formas espaciais que estudam o comportamento das superfícies com curvatura média ou curvatura Gaussiana constante. O estudo das superfícies regradas em $\mathbb{Q}^{3}(c)$ com curvatura média constante pode ser encontrado nos trabalhos [BDJ] e [BD], os quais são sempre citados quando o assunto envolve regradas com curvatura média constante. Em relação ao estudo das superfícies regradas em $\mathbb{S}_{1}^{3}$ não encontramos nada na literatura.

A seguir mostramos dois resultados, provavelmente conhecidos, que caracterizam as superfícies regradas em $\mathbb{Q}^{3}(c)$ e $\mathbb{S}_{1}^{3}$ com curvatura Gaussiana constante.

Proposição 2.1 Seja $M^{2} \subset \mathbb{Q}^{3}(c)$ uma superfície regrada com curvatura Gaussiana constante. Se

1. $c=0$, então $K=0$;

2. $c=1$, então $K=0$ ou $K=1$;

3. $c=-1$, então $K=-1$;

\section{Demonstração:}

Caso $c=0$ : Ver $[\mathrm{St}]$

Caso $c=1$ : Segue-se por (2.11) que $K$ é constante se, e somente se, $\frac{Q}{\left|X_{s}\right|^{2}}$ for constante. Se $Q \equiv 0$, então $K \equiv 1$. Se $\frac{Q}{\left|X_{s}\right|^{2}}=a$, onde $a$ é uma constante real não nula, então

$$
\begin{aligned}
\left(\frac{Q}{\left|X_{s}\right|^{2}}\right)_{t}=0 & \Leftrightarrow 2 Q\left\langle X_{s t}, X_{s}\right\rangle=0 \\
& \Rightarrow 2\left\langle X_{s t}, X_{s}\right\rangle=0 \\
& \Rightarrow\left(\left|\dot{e}_{1}\right|^{2}-1\right) \sin 2 t+ \\
& +2\left\langle\dot{e}_{1}, \dot{e}_{0}\right\rangle \cos 2 t=0 \\
& \Rightarrow\left\{\begin{array}{l}
\left|\dot{e}_{1}\right|^{2}=1 \\
\left\langle\dot{e}_{0}, \dot{e}_{1}\right\rangle=0
\end{array}\right. \\
& \Rightarrow K=0,
\end{aligned}
$$

onde a última implicação segue-se de (2.2) e (2.12). 
Caso $c=-1$ : Analogamente ao caso anterior, segue-se por (2.11) que $K$ é constante se, e somente se, $\frac{Q}{\left|X_{s}\right|^{2}}$ for constante. Se $Q \equiv 0$, então $K \equiv-1$. Se $\frac{Q}{\left|X_{s}\right|^{2}}=a$, onde $a$ é uma constante real não nula, então

$$
\begin{aligned}
\left(\frac{Q}{\left|X_{s}\right|^{2}}\right)_{t}=0 & \Leftrightarrow 2 Q\left\langle X_{s t}, X_{s}\right\rangle=0 \\
& \Rightarrow 2\left\langle X_{s t}, X_{s}\right\rangle=0 \\
& \Rightarrow\left(\left|\dot{e}_{1}\right|^{2}+1\right) \sinh 2 t+ \\
& +2\left\langle\dot{e}_{1}, \dot{e}_{0}\right\rangle \cosh 2 t=0 \\
& \Rightarrow\left\{\begin{array}{l}
\left|\dot{e}_{1}\right|^{2}=-1, \\
\left\langle\dot{e}_{0}, \dot{e}_{1}\right\rangle=0 .
\end{array}\right.
\end{aligned}
$$

Logo se $K$ é constante, então $K \equiv-1$, pois o vetor $\dot{e}_{1}(s)$ pertence ao espaço tangente a $\mathbb{H}^{3}, \forall s$, isto é, $\left|\dot{e}_{1}(s)\right|^{2}>0, \forall s$.

Proposição 2.2 Se $M^{2} \subset \mathbb{S}_{1}^{3}$ é uma superfície regrada tipo espaço com curvatura Gaussiana constante, então $K=1$ ou $K=2$.

\section{Demonstração:}

Segue-se por (2.18) que $K$ é constante se, e somente se, $\frac{Q}{P}$ for constante. Se $Q \equiv 0$, então $K \equiv 1$. Se $\frac{Q}{P}=a$, onde $a$ é uma constante real não nula, então

$$
\begin{aligned}
\left(\frac{Q}{P}\right)_{t}=0 & \Leftrightarrow P_{t} Q=0 \\
& \Rightarrow(B-A) \sin 2 t=0 \\
& \Rightarrow B=A .
\end{aligned}
$$

Logo por (2.17) e (2.19) temos que $a=1$, isto é, $K=2$.

\subsubsection{Superfícies regradas desenvolvíveis em $\mathbb{Q}^{3}(c)$}

Seja $X(s, t)=\alpha(s)+t \beta(s)$ a parametrização local de uma superfície regrada em $\mathbb{R}^{3}$, tal que $|\beta|=|\dot{\beta}|=1 \mathrm{e}\langle\dot{\alpha}, \dot{\beta}\rangle=0$. A superfície regrada $X(s, t)$ é dita desenvolvível se o campo $\eta$, normal à superfície, é constante ao longo das folhas, isto é, se $\eta_{t}$ é nulo. Não é difícil mostrar que as superfícies regradas desenvolvíveis em $\mathbb{R}^{3}$ são exatamente as superfícies regradas flat, melhor dizendo, uma superfície 
regrada em $\mathbb{R}^{3}$ é desenvolvível se, e somente se, a curvatura Gaussiana da superfície é identicamente nula.

Segue-se da expressão da curvatura Gaussiana de uma superfície regrada em $\mathbb{R}^{3}$ que a superfície é flat se, e somente se, os vetores $\dot{\alpha}, \beta, \dot{\beta}$ são linearmente dependentes em todo ponto. Analisando estes vetores não é difícil verificar que localmente uma superfície regrada flat é um pedaço de um cilindro, de um cone, ou de uma superfície tangente.

A seguir definiremos o conceito de superfície desenvolvível em $\mathbb{S}^{3}$ e $\mathbb{H}^{3}$ e estudaremos a extensão de alguns dos resultados anteriores. Analogamente ao caso Euclidiano, temos a seguinte definição:

Definição 2.3 Seja $M^{2}$ uma superfície regrada em $\mathbb{Q}^{3}(c), c= \pm 1$. Dizemos que $M^{2}$ é uma superfície desenvolvivel se o campo normal à superfície é paralelo ao longo das folhas.

Segue-se da definição anterior o seguinte resultado

Proposição 2.4 Seja $M^{2}$ uma superfície regrada em $\mathbb{Q}^{3}(c), c= \pm 1$. Então $M^{2}$ é uma superfície desenvolvivel se, e somente se, $K_{\text {int }}=c$, onde $K_{\text {int }}$ é a curvatura intrínseca da superficie.

\section{Demonstração:}

Faremos a demonstração apenas para o caso $c=1$, visto que a demonstração para o caso $c=-1$ é análoga, guardadas as particularidades de cada ambiente.

Sejam $M^{2}$ uma superfície regrada em $\mathbb{S}^{3}$, parametrizada como em (2.1), e $\eta=$ $X_{t} \wedge \frac{X_{s}}{\left|X_{s}\right|} \wedge X$ o campo unitário normal à superfície em $\mathbb{S}^{3}$. Se $M^{2}$ é desenvolvivel, então $\bar{\nabla}_{\dot{\gamma}(t)} \eta=0$, onde $\gamma(t)=X\left(s_{0}, t\right)$ é uma folha da superfície regrada e $\bar{\nabla}$ é a conexão de Levi-Civita em $\mathbb{S}^{3}$. Mas

$$
\bar{\nabla}_{\dot{\gamma}(t)} \eta=\eta_{t}-\left\langle\eta_{t}, X\right\rangle X=\eta_{t},
$$

pois

$$
\eta_{t}=X_{t} \wedge\left(\frac{X_{s t}\left|X_{s}\right|-\left(\left|X_{s}\right|\right)_{t} X_{s}}{\left|X_{s}\right|^{2}}\right) \wedge X
$$

Segue-se, da expressão de $\eta_{t}$ e do fato que $\eta$ é um campo unitário, que $\eta_{t}$ pertence ao espaço tangente à superfície, donde temos por (2.10) que

$$
\begin{aligned}
\eta_{t}= & \left\langle\eta_{t}, X_{t}\right\rangle X_{t}+\left\langle\eta_{t}, X_{s}\right\rangle \frac{X_{s}}{\left|X_{s}\right|^{2}} \\
& =\left\langle X_{t} \wedge X_{s t} \wedge X, X_{s}\right\rangle \frac{X_{s}}{\left|X_{s}\right|^{3}} \\
& =\frac{Q}{\left|X_{s}\right|^{3}} .
\end{aligned}
$$


Logo $\bar{\nabla}_{\dot{\gamma}(t)} \eta=0$ se, e somente se, $Q=0$, donde segue-se por (2.11) o desejado.

A construção de exemplos de superfícies regradas com $K=1$ e $K=-1$ em $\mathbb{S}^{3}$ e $\mathbb{H}^{3}$, respectivamente, é trivial de acordo com a definição e o teorema que passamos a descrever.

Definição 2.5 Um homeomorfismo $\phi: M_{1} \rightarrow M_{2}$ é chamado uma aplicação geodésica se para toda geodésica $\gamma \in M_{1}, \phi \circ \gamma$ é uma reparametrização de uma geodésica de $\mathrm{M}_{2}$.

Temos então o seguinte resultado, cuja demonstração encontra-se em [S].

Teorema 2.6 Sejam $\phi: \mathbb{Q}^{m}(c) \rightarrow \mathbb{R}^{m}$ uma aplicação geodésica e $M^{2} \subset \mathbb{Q}^{m}(c)$ uma superfície regrada. Então $M^{2}$ tem curvatura intrínseca $c$ se, e somente se, $\phi\left(M^{2}\right) \subset \mathbb{R}^{m}$ é uma superfície flat.

Segue-se assim que as superfícies com $K=1$ e $K=-1$ em $\mathbb{S}^{3}$ e $\mathbb{H}^{3}$, respectivamente, são localmente a imagem inversa através de uma aplicação geodésica de um cone, de um cilindro ou de uma superfície tangente. Vejamos qual a parametrização de uma aplicação geodésica de $\mathbb{S}^{3}$ e $\mathbb{H}^{3}$ em $\mathbb{R}^{3}$.

No caso esférico, definimos uma aplicação $\phi$ que associa a cada ponto $p$, pertencente a $\mathbb{S}_{+}^{3}=\left\{p=\left(p_{1}, p_{2}, p_{3}, p_{4}\right) \in \mathbb{R}^{4} / p_{4}>0\right\}$, ao ponto obtido pela intersecção de $\mathbb{R}^{3} \equiv \mathbb{R}^{3} \times\{1\} \subset \mathbb{R}^{4}$ com a reta que passa pela origem de $\mathbb{R}^{4}$ e $p$. É fácil verificar que $\phi: \mathbb{S}_{+}^{3} \rightarrow \mathbb{R}^{3}$ é uma aplicação geodésica, pois as geodésicas de $\mathbb{S}^{3}$ são determinadas pela interseç̧ão de $\mathbb{S}^{3}$ com planos que passam pela origem de $\mathbb{S}^{3}$. Segue-se ainda que $\phi$ é dada por

$$
\begin{aligned}
\phi: & \mathbb{S}_{+}^{3} \longrightarrow \mathbb{R}^{3} \equiv \mathbb{R}^{3} \times\{1\} \\
& p \longrightarrow\left(\frac{p_{1}}{p_{4}}, \frac{p_{2}}{p_{4}}, \frac{p_{3}}{p_{4}}, 1\right)
\end{aligned}
$$

e

$$
\begin{aligned}
\phi^{-1}: & \mathbb{R}^{3} \longrightarrow \mathbb{S}^{3}\left(\mathbb{S}_{+}^{3}\right) \\
& y=\left(y_{1}, y_{2}, y_{3}\right) \longrightarrow \frac{1}{\sqrt{1+||^{2}}}\left(y_{1}, y_{2}, y_{3}, 1\right) .
\end{aligned}
$$

De forma análoga, podemos definir uma aplicação geodésica no caso hiperbólico, com a diferença que neste caso teremos uma aplicação definida em todo $\mathbb{H}^{3}$. Seja $\phi$ a aplicação que associa a cada ponto $p \in \mathbb{H}^{3}$, o ponto obtido pela intersecção de $\mathbb{R}^{3} \equiv\{1\} \times \mathbb{R}^{3} \subset \mathbb{R}^{4}$ com a reta que passa pela origem de $\mathbb{R}^{4}$ e $p$. Observe que 


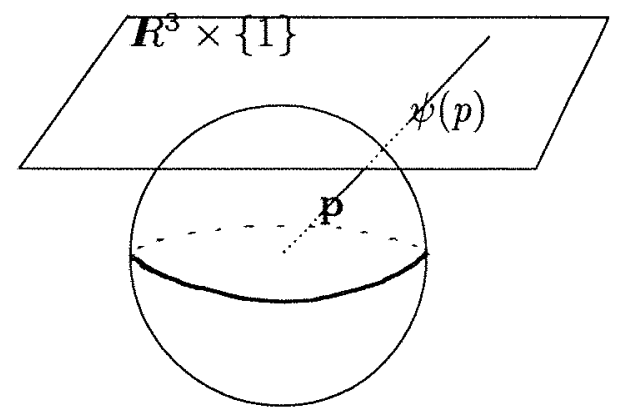

Figura 2.1: Aplicação geodésica no caso esférico

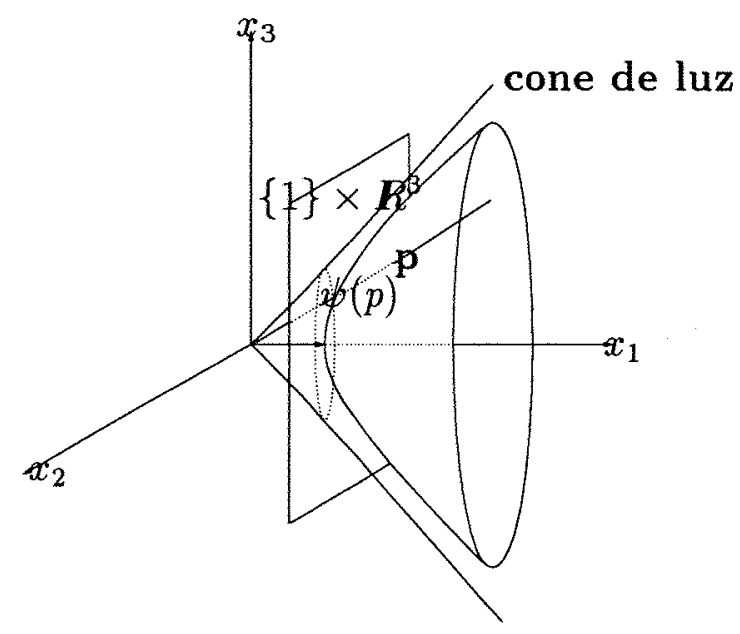

Figura 2.2: Aplicação geodésica no caso hiperbólico 
neste caso a imagem de $\mathbb{H}^{3}$ é a bola aberta de $\mathbb{R}^{3}$ limitada pela intersecção de $\mathbb{R}^{3}$ com o cone $-p_{1}^{2}+\sum_{j=2}^{4} p_{j}^{2}=0$. Segue-se ainda que $\phi$ é dada por

$$
\begin{aligned}
\phi: & \mathbb{H}^{3} \longrightarrow \mathbb{R}^{3} \equiv\{1\} \times \mathbb{R}^{3} \\
& p \longrightarrow\left(1, \frac{p_{2}}{p_{1}}, \frac{p_{3}}{p_{1}}, \frac{p_{4}}{p_{1}}\right)
\end{aligned}
$$

e

$$
\begin{aligned}
\phi^{-1}: & \mathbb{R}^{3} \longrightarrow \mathbb{H}^{3} \\
& y=\left(y_{2}, y_{3}, y_{4}\right) \longrightarrow \frac{1}{\sqrt{1+||^{2}}}\left(1, y_{2}, y_{3}, y_{4}\right) .
\end{aligned}
$$

\subsubsection{Superfícies regradas tipo espaço desenvolvíveis em $\mathbb{S}_{1}^{3}$}

Analogamente ao caso Riemanniano, podemos definir em $\mathbb{S}_{1}^{3}$ a noção de superfície desenvolvivel:

Definição 2.7 Seja $M^{2}$ uma superfície regrada tipo espaço em $\mathbb{S}_{1}^{3}$. Dizemos que $M^{2}$ é uma superfície desenvolvível se o campo normal à superfície é paralelo ao longo das folhas.

Segue-se da definição anterior o seguinte resultado:

Proposição 2.8 Seja $M^{2}$ uma superfície regrada tipo espaço em $\mathbb{S}_{1}^{3}$. Então $M^{2} e^{\prime}$ uma superfície desenvolvivel se, e somente se, $K_{\text {int }}=1$.

\section{Demonstração:}

É análoga a demonstração feita na Proposição (2.4). Observe que sendo $M^{2}$ uma superfície tipo espaço, o campo normal ao longo da superfície, $\eta=X_{t} \wedge \frac{\tilde{X}_{s}}{\left|\tilde{X}_{s}\right|} \wedge X$, determina em cada ponto de $M^{2}$ um vetor tipo tempo, isto é, $\langle\eta, \eta\rangle=-1$. 


\section{Capítulo 3}

\section{Superfícies regradas helicoidais em formas espaciais}

O objetivo deste capítulo é caracterizar as superfícies regradas helicoidais em $\mathbb{Q}^{3}(c)$, isto é, encontrar uma condição necessária e suficiente para que uma superfície regrada seja helicoidal. Tal caracterização é possível pois segue-se da Proposição (3.2) uma caracterização das hélices em $\mathbb{Q}^{3}(c)$. O resultado da Proposição (3.2) provavelmente é bem conhecido, no entanto, o único lugar que encontramos referência a ele, foi em [BDJ], mas que cuja notação não é adequada aos nossos objetivos. $\mathrm{Na}$ seção 3.1 caracterizaremos as hélices em $\mathbb{Q}^{3}(c)$ e na seção 3.2 caracterizaremos as superfícies regradas helicoidais em $\mathbb{Q}^{3}(c)$.

\subsection{Uma caracterização das hélices em formas es- paciais}

Utilizando as definições e notações dadas nas seções (1.4) e (1.5) temos que.

Definição 3.1 Uma curva $\gamma$ em $\mathbb{Q}^{3}(c)$ é dita uma hélice se possui curvatura e torção constantes não nulas.

Note que pela definição anterior, as curvas planas e as geodésicas do ambiente não estão sendo consideradas. Além disso, observamos que as hélices formam uma 
classe particular dentro da classe das curvas denominadas hélices generalizadas. Ver $[\mathrm{Ba}]$.

Proposição 3.2 Seja $\gamma: I \subset \mathbb{R} \longrightarrow \mathbb{Q}^{3}(c)$ uma curva parametrizada regular em $\mathbb{Q}^{3}(c)$ com $c= \pm 1$. Entâo $\gamma(I)$ é invariante por um movimento helicoidal se, $e$ somente se, $\gamma$ é uma hélice.

\section{Demonstração:}

Caso $c=-1$ :

Seja $\gamma: I \longrightarrow \mathbb{H}^{3}$ uma curva invariante por um movimento helicoidal. Podemos assumir, a menos de isometria de $\mathbb{H}^{3}$, que $\mathcal{P}^{2}=\left\{x \in \mathbb{H}^{3}: x_{2}=0\right\}$ é a 2-subvariedade totalmente geodésica de $\mathbb{H}^{3}$ ortogonal ao eixo invariante $\Gamma(r)=(\cosh r, \sinh r, 0,0)$. Claramente $\gamma(I)$ é a órbita de um ponto em $\mathcal{P}^{2}$, suponhamos $p=(x, 0, z, w)$, com $x \neq 1$. Temos então que a reparametrização por comprimento de arco de $\gamma$ é

$$
\gamma(s)=\left(x \cosh \frac{h s}{\theta}, x \sinh \frac{h s}{\theta}, z \cos \frac{s}{\theta}+w \sin \frac{s}{\theta},-z \sin \frac{s}{\theta}+w \cos \frac{s}{\theta}\right),
$$

$\operatorname{com} \theta=\sqrt{x^{2} h^{2}+z^{2}+w^{2}}$.

É imediato verificar que

$$
\begin{gathered}
\dot{\gamma}(s)=\left(\frac{h x}{\theta} \sinh \frac{h s}{\theta}, \frac{h x}{\theta} \cosh \frac{h s}{\theta},-\frac{z}{\theta} \sin \frac{s}{\theta}+\frac{w}{\theta} \cos \frac{s}{\theta},-\frac{z}{\theta} \cos \frac{s}{\theta}-\frac{w}{\theta} \sin \frac{s}{\theta}\right), \\
\ddot{\gamma}(s)=\left(\frac{h^{2} x}{\theta^{2}} \cosh \frac{h s}{\theta}, \frac{h^{2} x}{\theta^{2}} \sinh \frac{h s}{\theta},-\frac{z}{\theta^{2}} \cos \frac{s}{\theta}-\frac{w}{\theta^{2}} \sin \frac{s}{\theta}, \frac{z}{\theta^{2}} \sin \frac{s}{\theta}-\frac{w}{\theta^{2}} \cos \frac{s}{\theta}\right), \\
\dddot{\gamma}(s)=\left(\frac{h^{3} x}{\theta^{3}} \sinh \frac{h s}{\theta}, \frac{h^{3} x}{\theta^{3}} \cosh \frac{h s}{\theta}, \frac{z}{\theta^{3}} \sin \frac{s}{\theta}-\frac{w}{\theta^{3}} \cos \frac{s}{\theta}, \frac{z}{\theta^{3}} \cos \frac{s}{\theta}+\frac{w}{\theta^{3}} \sin \frac{s}{\theta}\right) .
\end{gathered}
$$

Então por (1.6) temos que

$$
\begin{aligned}
\kappa^{2} & =|\ddot{\gamma}|^{2}+1 \\
& =\frac{-x^{2} h^{4}+z^{2}+w^{2}}{\theta^{4}}+1 \\
& =\frac{\left(x^{2}-1\right)\left(1+h^{2}\right)^{2} x^{2}}{\left(x^{2}\left(h^{2}+1\right)-1\right)^{2}} .
\end{aligned}
$$

Além disso, após vários cálculos segue-se que

$$
\dot{\gamma} \wedge \dddot{\gamma} \wedge \gamma=\left\{\frac{h\left(1+h^{2}\right) x\left(w^{2}+z^{2}\right) \cosh \frac{h s}{\theta}}{\theta^{4}},\right.
$$




$$
\begin{aligned}
& \frac{h\left(1+h^{2}\right) x\left(w^{2}+z^{2}\right) \sinh \frac{h s}{\theta}}{\theta^{4}}, \\
& \frac{h\left(1+h^{2}\right) x^{2}\left(z \cos \frac{s}{\theta}+w \sin \frac{s}{\theta}\right)}{\theta^{4}}, \\
& \left.\frac{h\left(1+h^{2}\right) x^{2}\left(w \cos \frac{s}{\theta}-z \sin \frac{s}{\theta}\right)}{\theta^{4}}\right\},
\end{aligned}
$$

e portanto por (1.7) temos que

$$
\begin{aligned}
\tau & =-\frac{1}{\kappa^{2}}\left(\frac{-h\left(1+h^{2}\right)^{2} x^{2}\left(w^{2}+z^{2}\right)}{\left(w^{2}+z^{2}+x^{2} h^{2}\right)^{3}}\right) \\
& =\frac{h}{w^{2}+z^{2}+x^{2} h^{2}} .
\end{aligned}
$$

Observe que $\gamma$ nunca será uma curva plana, pois $\tau=0$ se, e somente se, $h=0$.

Segue-se portanto de (3.1) e (3.2) que toda curva invariante por um movimento helicoidal é uma hélice, pois suas funções curvatura e torção são constantes não nulas.

Reciprocamente, usando-se o fato que duas curvas com mesma curvatura e mesma torção são congruente em $\mathbb{H}^{3}$, queremos, dada uma hélice em $\mathbb{H}^{3}$, isto é, dados $\kappa>0$ e $\tau \neq 0$, determinar um ponto $p \in \mathcal{P}^{2}$ e um passo $h$, tal que a órbita de $p$ por $\mathcal{H}_{\varphi}^{h, 1}$ possui $\kappa$ e $\tau$ como curvatura e torção, respectivamente.

Como $-x^{2}+w^{2}+z^{2}=-1$, segue-se por (3.2) que

$$
w^{2}+z^{2}=\frac{h(1-h \tau)}{\tau\left(1+h^{2}\right)}
$$

e substituindo esta igualdade em (3.1), temos

$$
h=\frac{-\left(\kappa^{2}+\tau^{2}-1\right) \pm \sqrt{\left(\kappa^{2}+\tau^{2}-1\right)^{2}+4 \tau^{2}}}{2 \tau} .
$$

Observamos que como $\left(\kappa^{2}(s)+\tau^{2}(s)-1\right)>0, \forall s$, e por (3.2) $\tau$ e $h$ possuem o mesmo sinal, existe apenas uma possibilidade para $h$, a saber

$$
h=\frac{-\left(\kappa^{2}+\tau^{2}-1\right)+\sqrt{\left(\kappa^{2}+\tau^{2}-1\right)^{2}+4 \tau^{2}}}{2 \tau} .
$$

Portanto, segue-se diretamente de (3.3) e (3.4), que dada uma hélice é possível determinar um ponto $p=(x, 0, z, w)$ e um passo $h$ tal que a órbita de $p$ possui a mesma curvatura e torção que a hélice dada, donde segue-se o desejado. Observamos 
que na realidade determina-se não apenas um ponto, mas uma curva.

Caso $c=1$ :

A demonstração da primeira parte é análoga ao caso anterior, isto é, dados $\gamma$ : $I \longrightarrow \mathbb{S}^{3}$ uma curva invariante por um movimento helicoidal, $\mathcal{P}^{2}=\left\{y \in \mathbb{S}^{3}: y_{2}=0\right\}$ uma 2-subvariedade totalmente geodésica de $\mathbb{S}^{3}$ ortogonal ao eixo invariante $\Gamma(r)=$ $(\cos r, \sin r, 0,0)$ e $p=(x, 0, z, w) \in \mathcal{P}_{+}^{2}=\left\{y \in \mathcal{P}^{2}: y_{1}>0\right\}$ com $x \neq 1$, então

$$
\kappa^{2}=\frac{\left(1-x^{2}\right)\left(h^{2}-1\right)^{2} x^{2}}{\left(x^{2}\left(h^{2}-1\right)+1\right)^{2}}, \quad \tau=-\frac{h}{x^{2}\left(h^{2}-1\right)+1} .
$$

Temos que $\kappa=0$ se, e somente se, $h= \pm 1$, mas neste caso a órbita de qualquer ponto em $\mathcal{P}_{+}^{2}$ se degenera em um grande círculo de $S^{3}$. Consideremos então $h \neq \pm 1$. Observe ainda que $\tau=0$ se, e somente se, $h=0$.

$\mathrm{Na}$ demonstração da segunda parte existe uma diferença. Analogamente ao caso anterior, é fácil verificar que

$$
x^{2}=\frac{-(h+\tau)}{\tau\left(h^{2}-1\right)}, \quad h=\frac{-\left(\kappa^{2}+\tau^{2}+1\right) \pm \sqrt{\left(\kappa^{2}+\tau^{2}+1\right)^{2}-4 \tau^{2}}}{2 \tau} .
$$

No entanto, não é possível descartarmos uma das alternativas para $h$, pois para todo $s$ vale que $\sqrt{\left(\kappa^{2}+\tau^{2}+1\right)^{2}-4 \tau^{2}}<\left(\kappa^{2}+\tau^{2}+1\right)$.

Segue-se então que neste caso é possível determinar dois passos, e portanto dois pontos em $\mathcal{P}_{+}^{2}$, cada um associado a um passo. $\mathrm{Na}$ realidade determinamos duas curvas. É possível, no entanto, verificar com cálculos diretos que as respectivas funções curvatura são iguais e portanto as duas órbitas encontradas são curvas congruentes em $\mathbb{R}^{4}$, donde segue-se que são curvas congruentes em $S^{3}$.

Uma simples, mas importante, conseqüência da proposição anterior é que toda hélice pode ser parametrizada como a órbita de um ponto $p \in \mathcal{P}^{2}, \mathcal{P}_{+}^{2}$ no caso $c=1$, e tem, a menos de uma isometria de $\mathbb{Q}^{3}(c)$, a seguinte expressão:

$$
\begin{array}{ll}
\gamma(s)=\left(x \cosh \frac{h s}{\theta}, x \sinh \frac{h s}{\theta}, z \cos \frac{s}{\theta},-z \sin \frac{s}{\theta}\right), & \text { se } c=-1, \\
\gamma(s)=\left(x \cos \frac{s}{\theta_{1}},-x \sin \frac{s}{\theta_{1}}, \frac{h s}{\theta_{1}}\right), & \text { se } c=0, \\
\gamma(s)=\left(x \cos \frac{h s}{\theta},-x \sin \frac{h s}{\theta}, z \cos \frac{s}{\theta},-z \sin \frac{s}{\theta}\right), & \text { se } c=1,
\end{array}
$$

onde $\theta=\sqrt{x^{2} h^{2}+z^{2}}$ e $\theta_{1}=\sqrt{x^{2}+h^{2}}$. O caso $c=0$ não foi feito na proposição anterior, pois pode ser facilmente encontrado nos livros de geometria básica. 


\subsection{Uma caracterização das superfícies regradas helicoidais em formas espaciais}

Mostraremos no próximo teorema, que uma condição suficiente para que uma superfície regrada seja helicoidal, é que suas curvaturas Gaussiana e média sejam constantes em relação ao parâmetro translacional.

Teorema 3.3 Seja $X(s, t)$ a parametrização local de uma superfície regrada em $\mathbb{Q}^{3}(c)$ dada por (2.1). Se $X(s, t)$ possui curvatura Gaussiana e curvatura média constantes em relação ao parâmetro translacional, entâo $X(s, t)$ é a parametrização de uma superfície regrada helicoidal.

\section{Demonstração:}

Caso $c=0$ :

Tomando-se a base ortonormal $\mathcal{B}=\left\{e_{1}, \dot{e}_{1}, e_{1} \times \dot{e}_{1}\right\}$ de $\mathbb{R}^{3}$ e usando-se a notação dada em (2.3), segue-se por (1.8) que

$$
\kappa_{e_{1}}^{2}=1+J^{2}, \quad \tau_{e_{1}}=\frac{\dot{J}}{1+J^{2}},
$$

onde $\kappa_{e_{1}}$ e $\tau_{e_{1}}$ denotam, respectivamente, a curvatura e a torção da curva $e_{1}$.

Por hipótese e usando-se (2.4), temos

$$
\begin{aligned}
K_{s}=0 & \Leftrightarrow \quad\left(\frac{Q}{D^{2}}\right)_{s}=0 \\
& \Leftrightarrow \quad \dot{Q} t^{2}-\dot{Q} Q^{2}=0 \\
& \Rightarrow Q=c t e .
\end{aligned}
$$

Como $Q$ é função constante segue-se então que

$$
\begin{aligned}
H_{s}=0 & \Leftrightarrow\left(\frac{J t^{2}+Q(Q J-F)}{2 D^{3}}\right)_{s}=0 \\
& \Leftrightarrow \quad \dot{J} t^{2}+Q(Q \dot{J}-\dot{F})=0 \\
& \Rightarrow\left\{\begin{array}{l}
J=\text { cte } \\
F=\text { cte. }
\end{array}\right.
\end{aligned}
$$

Segue-se então de (3.6) e (3.7) que $e_{1}$ é uma curva esférica plana, isto é, está contida na interseç̧ão de $\mathbb{S}^{2}$ com um plano de $\mathbb{R}^{3}$. Observamos que este plano conterá a origem se, e somente se, $J=0$. 
Sem perda de generalidade, podemos supor que a curva $e_{1}$ está contida no plano $x y$, isto é,

$$
e_{1}(s)=\left(d \sin s,-d \cos s, \sqrt{1-d^{2}}\right),
$$

onde $d$ é uma constante real não nula. Usando-se a base $\mathcal{B}$, é fácil verificar que $\dot{e}_{0}=F e_{1}+Q e_{1} \times \dot{e}_{1} \mathrm{e}$, usando-se o fato que

$$
e_{1} \times \dot{e}_{1}=\left(-d \sqrt{1-d^{2}} \sin s, d \sqrt{1-d^{2}} \cos s, d^{2}\right),
$$

temos

$$
e_{0}(s)=(a \cos s, a \sin s, b s),
$$

onde $a, b$ são constantes reais, que dependem do valor das constantes $F, Q$, e $d$.

Logo $X(s, t)=e_{0}(s)+t e_{1}(s)$ é a parametrização local de uma superfície regrada helicoidal em $\mathbb{R}^{3}$.

\section{Caso $c=-1$ :}

Mostraremos, em primeiro lugar, que a curva $e_{0}$ é uma hélice em $\mathbb{H}^{3}$, isto é, $\kappa_{e_{0}}$ e $\tau_{e_{0}}$ são funções constantes.

Como $e_{0}$ é uma curva parametrizada pelo comprimento de arco, segue-se respectivamente de (1.6) e (1.7) que

$$
\kappa_{e_{0}}=\left|\ddot{e}_{0}-e_{0}\right|, \quad \tau_{e_{0}}=\frac{-1}{\kappa_{e_{0}}^{2}}\left\langle\dot{e}_{0} \wedge \dddot{e}_{0} \wedge e_{0}, \ddot{e}_{0}\right\rangle .
$$

Tomando-se a base ortonormal $\left\{\dot{e}_{0}, e_{1}, \dot{e}_{0} \wedge e_{1} \wedge e_{0}, e_{0}\right\}$ de $\mathbb{L}^{4}$ ao longo de $e_{0}$, e utilizando-se a notação dada em (2.10), é fácil verificar que

$$
\ddot{e}_{0}=-\left\langle\dot{e}_{0}, \dot{e}_{1}\right\rangle e_{1}-F \dot{e}_{0} \wedge e_{1} \wedge e_{0}+e_{0},
$$

donde segue-se que

$$
\kappa_{e_{0}}^{2}=\left\langle\dot{e}_{0}, \dot{e}_{1}\right\rangle^{2}+F^{2}
$$

Temos ainda que

$$
\dot{F}=\left\langle\dddot{e}_{0}, e_{1} \wedge \dot{e}_{0} \wedge e_{0}\right\rangle+\left\langle\ddot{e}_{0}, \dot{e}_{1} \wedge \dot{e}_{0} \wedge e_{0}\right\rangle,
$$

mas pela expressão de $\ddot{e}_{0}$

$$
\begin{aligned}
\left\langle\ddot{e}_{0}, \dot{e}_{1} \wedge \dot{e}_{0} \wedge e_{0}\right\rangle & =-\left\langle\dot{e}_{0}, \dot{e}_{1}\right\rangle\left\langle e_{1}, \dot{e}_{1} \wedge \dot{e}_{0} \wedge e_{0}\right\rangle-F\left\langle\dot{e}_{0} \wedge e_{1} \wedge e_{0}, \dot{e}_{1} \wedge \dot{e}_{0} \wedge e_{0}\right\rangle \\
& =\left\langle\dot{e}_{0}, \dot{e}_{1}\right\rangle Q
\end{aligned}
$$


donde temos que,

$$
\dot{F}=\left\langle\dddot{e}_{0}, e_{1} \wedge \dot{e}_{0} \wedge e_{0}\right\rangle+\left\langle\dot{e}_{0}, \dot{e}_{1}\right\rangle Q .
$$

Portanto mostra-se que

$$
\begin{aligned}
\left\langle\dot{e}_{0} \wedge \dddot{e}_{0} \wedge e_{0}, \ddot{e}_{0}\right\rangle=-\left\langle\dot{e}_{0}, \dot{e}_{1}\right\rangle\left\langle e_{1}, \dot{e}_{0} \wedge \dddot{e}_{0} \wedge e_{0}\right\rangle+ \\
-F\left\langle\dot{e}_{0} \wedge \dddot{e}_{0} \wedge e_{0}, \dot{e}_{0} \wedge e_{1} \wedge e_{0}\right\rangle \\
=-\left\langle\dot{e}_{0}, \dot{e}_{1}\right\rangle\left(\dot{F}-\left\langle\dot{e}_{0}, \dot{e}_{1}\right\rangle Q\right)+ \\
\left.+F\left(2\left\langle\ddot{e}_{0}, \dot{e}_{1}\right\rangle+\left\langle\dot{e}_{1}, \ddot{e}_{0}\right\rangle\right)\right)
\end{aligned}
$$

donde segue-se que,

$$
\tau_{e_{0}}=\frac{-1}{\kappa_{e_{0}}^{2}}\left(-\left\langle\dot{e}_{0}, \dot{e}_{1}\right\rangle\left(\dot{F}-\left\langle\dot{e}_{0}, \dot{e}_{1}\right\rangle Q\right)+F\left(2\left\langle\ddot{e}_{0}, \dot{e}_{1}\right\rangle+\left\langle\dot{e}_{1}, \ddot{e}_{0}\right\rangle\right)\right) .
$$

Temos ainda por (2.11) que

$$
\begin{aligned}
K_{s}=0 \quad & \Leftrightarrow\left(\frac{Q}{\left|X_{s}\right|^{2}}\right)_{s}=0 \\
& \Leftrightarrow \quad \dot{Q}\left|X_{s}\right|^{2}-2\left\langle X_{s s}, X_{s}\right\rangle Q=0 .
\end{aligned}
$$

Em particular, para $t=0$, vale que

$$
\left\langle X_{s s}, X_{s}\right\rangle=0 \Rightarrow \dot{Q}=0
$$

donde segue-se que

$$
\left\langle X_{s s}, X_{s}\right\rangle=0 \forall s, t \Rightarrow\left\{\begin{array}{l}
\left|\dot{e}_{1}\right|^{2}=\text { cte } \\
\left\langle\dot{e}_{0}, \dot{e}_{1}\right\rangle=\text { cte }
\end{array}\right.
$$

isto é, $\left|X_{s}\right|^{2}$ não depende da variável $s$.

Usando-se (3.8) temos por (2.11) que

$$
\begin{aligned}
H_{s}=0 & \Leftrightarrow h_{s}=0 \\
& \Leftrightarrow \quad \dot{F} \cosh ^{2} t+\dot{D} \sinh ^{2} t+(\dot{G}+\dot{L}) \cosh t \sinh t=0 .
\end{aligned}
$$

Como $\cosh ^{2} t, \sinh ^{2} t$ e $\cosh t \sinh t$ são linearmente independentes, como funções de $t$, segue-se que $F, D$ e $(G+L)$ são funções constantes. Observando que $\dot{Q}=G-L=0$ e $\dot{G}+\dot{L}=0$ segue-se que $G$ e $L$ são funções constantes, o que completa a primeira parte da demonstração. 
Mostraremos agora que $X(s, t)$ é uma superfície regrada helicoidal em $\mathbb{H}^{3}$. Seja $T$ a isometria de $\mathbb{H}^{3}$ tal que $T\left(e_{0}\right)=\beta_{0}$, onde $\beta_{0}$ é a órbita do ponto $p=(x, 0, z, 0)$, isto é, $\beta_{0}$ é dada como em (3.5). Afirmamos que

$$
\begin{aligned}
Y(s, t)=T(X(s, t)) & =T\left(e_{0}\right) \cosh t+T\left(e_{1}\right) \sinh t \\
& =\beta_{0} \cosh t+\beta_{1} \sinh t
\end{aligned}
$$

é uma superfície regrada helicoidal.

Observamos que sendo $T$ uma isometria de $\mathbb{H}^{3}, Y(s, t)$ herda todas as propriedades geométricas de $X(s, t)$. É fácil ver que as curvas $\beta_{0}$ e $\beta_{1}$ têm as mesmas propriedades que as curvas $e_{0}$ e $e_{1}$. Além disso, segue-se que $Y(s, t)$ é uma superfície regrada com $H_{s}{ }^{Y}=0$ e $K_{s}{ }^{Y}=0$. Determinemos $\beta_{1}$.

Sabendo-se que $\beta_{1}(s) \in T_{\beta_{0}(s)} Y \forall s$ e denotando-se por $\left\{T_{\beta_{0}}, N_{\beta_{0}}, B_{\beta_{0}}\right\}$ o triedro de Frenet da curva $\beta_{0}$, não é difícil verificar, usando-se a observação anterior, que $\beta_{1}(s)=a N_{\beta_{0}}+b B_{\beta_{0}}$, onde

$$
a=\frac{\left\langle\beta_{1}, \ddot{\beta}_{0}-\beta_{0}\right\rangle}{\kappa_{\beta_{0}}}=-\frac{\left\langle\dot{\beta}_{0}, \dot{\beta}_{1}\right\rangle}{\kappa_{\beta_{0}}}=c t e
$$

$\mathrm{e}$

$$
b=\frac{\left\langle\beta_{1}, \dot{\beta}_{0} \wedge \ddot{\beta}_{0} \wedge \beta_{0}\right\rangle}{\kappa_{\beta_{0}}}=\frac{F}{\kappa_{\beta_{0}}}=c t e,
$$

com $a^{2}+b^{2}=1$. Após alguns cálculos, segue-se ainda que

$$
\begin{aligned}
N_{\beta_{0}}(s)= & \frac{\ddot{\beta}_{0}-\beta_{0}}{\kappa_{\beta_{0}}} \\
= & \frac{1}{\kappa}\left(\left(\frac{x h^{2}}{\theta^{2}}-x\right) \cosh \frac{h s}{\theta},\left(\frac{x h^{2}}{\theta^{2}}-x\right) \sinh \frac{h s}{\theta},\right. \\
& \left.-\left(\frac{z}{\theta^{2}}+z\right) \cos \frac{s}{\theta},\left(\frac{z}{\theta^{2}}+z\right) \sin \frac{s}{\theta}\right) \\
= & \left(-z \cosh \frac{h s}{\theta},-z \sinh \frac{h s}{\theta},-x \cos \frac{s}{\theta}, x \sin \frac{s}{\theta}\right),
\end{aligned}
$$

e

$$
\begin{aligned}
B_{\beta_{0}}(s) & =\frac{\dot{\beta}_{0} \wedge \ddot{\beta}_{0} \wedge \beta_{0}}{\kappa_{\beta_{0}}} \\
& =\frac{1+h^{2}}{\kappa \theta^{3}}\left(x z^{2} \sinh \frac{h s}{\theta}, x z^{2} \cosh \frac{h s}{\theta}, h x^{2} z \sin \frac{s}{\theta}, h x^{2} z \cos \frac{h s}{\theta}\right) \\
& =\left(\frac{z}{\theta} \sinh \frac{h s}{\theta}, \frac{z}{\theta} \cosh \frac{h s}{\theta}, \frac{h x}{\theta} \sin \frac{s}{\theta}, \frac{h x}{\theta} \cos \frac{h s}{\theta}\right) .
\end{aligned}
$$

Agora é fácil verificar que

$$
\beta_{1}(s)=\left(-a z \cosh \frac{h s}{\theta}+\frac{b z}{\theta} \sinh \frac{h s}{\theta},-a z \sinh \frac{h s}{\theta}+\frac{b z}{\theta} \cosh \frac{h s}{\theta},\right.
$$




$$
\left.-a x \cos \frac{s}{\theta}+\frac{b h x}{\theta} \sin \frac{s}{\theta}, a x \sin \frac{s}{\theta}+\frac{b h x}{\theta} \cos \frac{s}{\theta}\right)
$$

é uma curva invariante pelo mesmo movimento helicoidal que deixa $\beta_{0}$ invariante, isto é,

$$
\begin{aligned}
\mathcal{H}_{\varphi}\left(\beta_{1}(s)\right)= & \left(-a z \cosh \left(\frac{h s}{\theta}+h \varphi\right)+\frac{b z}{\theta} \sinh \left(\frac{h s}{\theta}+h \varphi\right),\right. \\
& -a z \sinh \left(\frac{h s}{\theta}+h \varphi\right)+\frac{b z}{\theta} \cosh \left(\frac{h s}{\theta}+h \varphi\right), \\
& -a x \cos \left(\frac{s}{\theta}+\varphi\right)+\frac{b h x}{\theta} \sin \left(\frac{s}{\theta}+\varphi\right) \\
& \left.a x \sin \left(\frac{s}{\theta}+\varphi\right)+\frac{b h x}{\theta} \cos \left(\frac{s}{\theta}+\varphi\right)\right)
\end{aligned}
$$

Logo $Y(s, t)$ é uma superfície regrada helicoidal, e portanto, pelo comentário feito após a Observação (1.17), temos que $X(s, t)$ também é uma superfície regrada helicoidal, como queríamos mostrar.

Caso $c=1$ :

A demonstração segue de forma análoga ao caso $c=-1$, guardadas as particularidades de cada ambiente.

Observando-se que pela Proposição (1.16), toda superfície helicoidal, em particular regrada helicoidal, possui curvatura Gaussiana e curvatura média constantes em relação a um dos parâmetros podemos fornecer uma recíproca para o teorema anterior.

Teorema 3.4 Seja $X$ a parametrização de uma superfície regrada em $\mathbb{Q}^{3}($ c). São equivalentes:

(i) X possui curvaturas Gaussiana e média constantes em relação ao parâmetro translacional;

(ii) X é a parametrização de uma superfície regrada helicoidal definida pelo movimento helicoidal de uma geodésica em $\mathbb{Q}^{3}(c)$.

Note que se $M^{2}$ é uma superfície regrada helicoidal em $\mathbb{Q}^{3}(c)$ definida pelo movimento helicoidal de uma geodésica, então a parametrização local de $M^{2}$ dada por $X(s, t)=\mathcal{H}_{s}(\gamma(t))$ é uma parametrização do tipo (2.1), onde $\gamma(t)$ é uma geodésica de $M^{2}$, e portanto de $\mathbb{Q}^{3}(c)$, e $\mathcal{H}_{s}$ é o movimento helicoidal definido no Teorema (1.14) que deixa $M^{2}$ invariante. Vejamos o que ocorre em cada caso.

- caso $c=0$ : 
Tomemos $\gamma(t)=a+t b$, onde $a=\left(a_{1}, a_{2}, a_{3}\right)$ e $b=\left(b_{1}, b_{2}, b_{3}\right)$ são vetores em $\mathbb{R}^{3}$ com $|b|^{2}=1$. Cálculos simples mostram que

$$
\begin{aligned}
X(s, t) & =\mathcal{H}_{s}(\gamma(t)) \\
& =\alpha(s)+t \beta(s),
\end{aligned}
$$

onde

$$
\alpha(s)=\left(a_{1} \cos s+a_{2} \sin s,-a_{1} \sin s+a_{2} \cos s, h s+a_{3}\right)
$$

e

$$
\beta(s)=\left(b_{1} \cos s+b_{2} \sin s,-b_{1} \sin s+b_{2} \cos s, b_{3}\right) .
$$

- caso $c=-1$ :

Tomemos $\gamma(t)=a \cosh t+b \sinh t$, onde $a=\left(a_{1}, a_{2}, a_{3}, a_{4}\right)$ e $b=\left(b_{1}, b_{2}, b_{3}, b_{4}\right)$ são vetores em $\mathbb{L}^{4}$ tais que $|a|^{2}=-1,|b|^{2}=1$ e $\langle a, b\rangle=0$. Após cálculos simples mostra-se que

$$
\begin{aligned}
X(s, t) & =\mathcal{H}_{s}(\gamma(t)) \\
& =\alpha(s) \cosh t+\beta(s) \sinh t
\end{aligned}
$$

onde

$$
\begin{array}{r}
\alpha(s)=\left(a_{1} \cosh h s+a_{2} \sinh h s, a_{1} \sinh h s+a_{2} \cosh h s,\right. \\
\left.a_{3} \cos s+a_{4} \sin s,-a_{3} \sin s+a_{4} \cos s\right)
\end{array}
$$

$\mathrm{e}$

$$
\begin{gathered}
\beta(s)=\left(b_{1} \cosh h s+b_{2} \sinh h s, b_{1} \sinh h s+b_{2} \cosh h s,\right. \\
\left.b_{3} \cos s+b_{4} \sin s,-b_{3} \sin s+b_{4} \cos s\right),
\end{gathered}
$$

$\operatorname{com}|\alpha(s)|^{2}=-1,|\beta(s)|^{2}=1$ e $\langle\alpha(s), \beta(s)\rangle=0$.

- caso $c=1$ :

Tomemos $\gamma(t)=a \cos t+b \sin t$, onde $a=\left(a_{1}, a_{2}, a_{3}, a_{4}\right)$ e $b=\left(b_{1}, b_{2}, b_{3}, b_{4}\right)$ são vetores em $\mathbb{R}^{4}$ tais que $|a|^{2}=|b|^{2}=1$ e $\langle a, b\rangle=0$. Analogamente, após cálculos simples mostra-se que

$$
\begin{aligned}
X(s, t) & =\mathcal{H}_{s}(\gamma(t)) \\
& =\alpha(s) \cos t+\beta(s) \sin t
\end{aligned}
$$

onde

$$
\begin{array}{r}
\alpha(s)=\left(a_{1} \cos h s+a_{2} \sin h s,-a_{1} \sin h s+a_{2} \cos h s\right. \\
\left.a_{3} \cos s+a_{4} \sin s,-a_{3} \sin s+a_{4} \cos s\right)
\end{array}
$$


e

$$
\begin{array}{r}
\beta(s)=\left(b_{1} \cos h s+b_{2} \sin h s,-b_{1} \sin h s+b_{2} \cos h s,\right. \\
\left.b_{3} \cos s+b_{4} \sin s,-b_{3} \sin s+b_{4} \cos s\right), \\
\operatorname{com}|\alpha(s)|^{2}=|\beta(s)|^{2}=1 \text { e }\langle\alpha(s), \beta(s)\rangle=0 .
\end{array}
$$




\section{Capítulo 4}

\section{Superfícies regradas e de Weingarten em formas espaciais}

Como já mencionado na introdução, generalizamos neste capítulo, a menos de uma hipótese técnica, um resultado obtido separadamente por Beltrami e Dini em $1865 / 66$ e mais tarde reescrito por Kühnel. Mais especificamente, classificamos as superfícies regradas de Weingarten $\mathrm{em} \mathbb{Q}^{3}(c)$. De acordo com a seção (1.8), para fazermos tal classificação, basta verificarmos quais são as superfícies regradas em $\mathbb{Q}^{3}(c)$ que satisfazem a relação $K_{t} H_{s}=K_{s} H_{t}$. Como as superfícies com curvatura Gaussiana constante ou curvatura média constante são exemplos de superfícies de Weingarten, não as estaremos considerando neste capítulo. Na seção (4.1) classificaremos as superfícies regradas de Weingarten em $\mathbb{S}^{3}$ e na seção (4.2) classificaremos as $\mathrm{em} \mathbb{H}^{3}$.

\subsection{Classificação das superfícies regradas e de Wein- garten em $\mathbb{S}^{3}$}

Para fazermos tal classificação, como já citado, basta verificarmos quais são as características que uma superfície deve ter, afim de que ela satisfaça a equação $H_{s} K_{t}-$ $H_{t} K_{s}=0$. No que se segue estaremos usando todas as definições e resultados dados nos Capítulos 2 e 3, com o objetivo de resolver tal equação.

Lema 4.1 Seja $M^{2} \subset \mathbb{S}^{3}$ uma superfície conexa regrada não desenvolvível e parame- 
trizada como em (2.1). Se a equação $K_{t} H_{s}-K_{s} H_{t}=0$ é satisfeita, então a função $\digamma(s)=\frac{1+\left|\dot{e}_{1}\right|^{2}}{Q}$ é constante.

\section{Demonstração:}

Como $M^{2}$ é uma superfície não desenvolvível, existe um ponto $p \in M^{2}$ tal que $K(p) \neq 1$, o que é equivalente a dizer que $Q\left(s_{0}\right) \neq 0$, onde $p=\left(s_{0}, t_{0}\right)$. Logo existe um intervalo $\tilde{V} \subset \mathbb{R}$ contendo $s_{0}$, tal que $Q(s) \neq 0 \forall s \in \tilde{V}$, ou equivalentemente, existe uma vizinhança $V \subset M^{2}$ de $p$ tal que $K(q) \neq 1 \forall q \in V$.

Após derivação direta de (2.11) segue-se que

$$
\left\{\begin{array}{l}
K_{s}=\frac{-2 Q}{\left|X_{s}\right|^{6}}\left(\dot{Q}\left|X_{s}\right|^{2}-2 Q\left\langle X_{s s}, X_{s}\right\rangle\right) \\
K_{t}=\frac{4 Q^{2}\left\langle X_{s t}, X_{s}\right\rangle}{\left|X_{s}\right|} \\
H_{s}=\frac{h_{s}\left|X_{s}\right|^{6}-3 h\left\langle X_{s s}, X_{s}\right\rangle}{2\left|X_{s}\right|^{5}} \\
H_{t}=\frac{h_{t}\left|X_{s}\right|^{2}-3 h\left\langle X_{s t}, X_{s}\right\rangle}{2\left|X_{s}\right|^{5}}
\end{array}\right.
$$

Então na vizinhança $V$ vale que

$$
\begin{aligned}
H_{s} K_{t}-H_{t} K_{s}=0 & \Leftrightarrow-h_{t}\left|X_{s}\right|^{2}\left(\dot{Q}\left|X_{s}\right|^{2}-2 Q\left\langle X_{s s}, X_{s}\right\rangle\right)+ \\
& -2 Q h_{s}\left|X_{s}\right|^{2}\left\langle X_{s t}, X_{s}\right\rangle+3 h \dot{Q}\left|X_{s}\right|^{2}\left\langle X_{s t}, X_{s}\right\rangle=0
\end{aligned}
$$

ou equivalentemente,

$$
-h_{t}\left|X_{s}\right|^{2}\left(\frac{Q}{\left|X_{s}\right|^{2}}\right)_{s}+h \dot{Q}\left\langle X_{s t}, X_{s}\right\rangle+\left(\left|X_{s}\right|^{2}\right)_{t} h^{2}\left(\frac{Q}{h}\right)_{s}=0
$$

O estudo das soluções da equação (4.2), da forma como ela esta apresentada, mostrou-se muito complicado devido a quantidade de funções envolvidas na mesma. A solução para este problema, foi encontrada substituindo algumas funções por sua expressão em termos da parametrização dada.

Após cálculos demorados, mas no entanto elementares, mostra-se que na vizinhança $V$ vale a equação

$$
\begin{aligned}
H_{s} K_{t}-H_{t} K_{s}=0 & \Leftrightarrow \frac{1}{2} Q\left|X_{s}\right|^{2} \mathcal{J}(s, t)=0 \\
& \Leftrightarrow \mathcal{J}(s, t)=0,
\end{aligned}
$$

onde

$$
\mathcal{J}(s, t)=g_{1}(s) \sin 2 t+g_{2}(s) \sin 4 t+g_{3}(s) \cos 2 t+g_{4}(s) \cos 4 t+g_{5}(s)
$$


com

$$
\begin{aligned}
& g_{1}(s)=-2 Q\left(\left(-1+\left|\dot{e}_{1}\right|^{2}\right) \dot{D}+(F-D)\left|\dot{e}_{1}\right|^{2}+\left(-1+\left|\dot{e}_{1}\right|^{2}\right) \dot{F}\right)+ \\
& +\left(D\left(-5+\left|\dot{e}_{1}\right|^{2}\right)+\left(-1+5\left|\dot{e}_{1}\right|^{2}\right) F\right) \dot{Q} \\
& g_{2}(s)=2 Q\left(\left(-1+\left|\dot{e}_{1}\right|^{2}\right) \dot{D}+2(G+L)\left\langle\dot{e}_{0}, \dot{e}_{1}\right\rangle+(F-D)\left|\dot{e}_{1}\right|^{2}-\right. \\
& \left.-\left(-1+\left|\dot{e}_{1}\right|^{2}\right) \dot{F}-2\left\langle\dot{e}_{0}, \dot{e}_{1}\right\rangle(\dot{G}+\dot{L})\right)+ \\
& +\left(-D\left(-1+\left|\dot{e}_{1}\right|^{2}\right)+\left(-1+\left|\dot{e}_{1}\right|^{2}\right) F+\right. \\
& \left.+2\left\langle\dot{e}_{0}, \dot{e}_{1}\right\rangle(G+L)\right) \dot{Q} \\
& g_{3}(s)=(G+L)\left(Q\left|\dot{e}_{1}\right|^{2}-\left(+1+\left|\dot{e}_{1}\right|^{2}\right) \dot{Q}\right)+\left\langle\dot{e}_{0}, \dot{e}_{1}\right\rangle(-2 Q(\dot{D}+\dot{F})+ \\
& +3(D+F) \dot{Q}) \\
& \begin{aligned}
g_{4}(s) & =2 Q\left(2(F-D)\left\langle\dot{e}_{0}, \dot{e}_{1}\right\rangle-(G+L)\left|\dot{e}_{1}\right|^{2}+2\left\langle\dot{e}_{0}, \dot{e}_{1}\right\rangle(\dot{D}-\right. \\
& \left.-\dot{F})+\left(-1+\left|\dot{e}_{1}\right|^{2}\right)(\dot{G}+\dot{L})\right)-\left(2\left\langle\dot{e}_{0}, \dot{e}_{1}\right\rangle(D-\right. \\
& \left.-F)+\left(-1+\left|\dot{e}_{1}\right|^{2}\right)(G+L)\right) \dot{Q}
\end{aligned} \\
& g_{5}(s)=2 Q\left(2(D-F)\left\langle\dot{e}_{0}, \dot{e}_{1}\right\rangle-(G+L)\left|\dot{e}_{1}\right|^{2}+2\left\langle\dot{e}_{0}, \dot{e}_{1}\right\rangle(\dot{D}-\right. \\
& \text { - } \left.\dot{F})-\left(-1+\left|\dot{e}_{1}\right|^{2}\right)(\dot{G}+\dot{L})\right)-5\left(2\left\langle\dot{e}_{0}, \dot{e}_{1}\right\rangle(D-\right. \\
& \left.-F)-\left(-1+\left|\dot{e}_{1}\right|^{2}\right)(G+L)\right) \dot{Q} \text {. }
\end{aligned}
$$

Segue-se então que na vizinhança de um ponto onde $K \neq 1$ a equação $K_{t} H_{s}-K_{s} H_{t}=$ 0 é satisfeita se, e somente se, $\mathcal{J}(s, t)=0, \forall s, t$. Observamos no entanto, que as funções $\sin 2 t, \sin 4 t, \cos 2 t$ e $\cos 4 t$ são linearmente independentes como funções de $t$, o que implica que a equação é satisfeita se, e somente se, $g_{1}(s)=g_{2}(s)=g_{3}(s)=$ $g_{4}(s)=g_{5}(s)=0$. Passemos então ao estudo destas cinco EDO's.

É fácil ver que as EDO's anteriores verificam a seguinte relação matricial

$$
[X]_{5 \times 5} \cdot[Y]_{5 \times 1}=[W]_{5 \times 1}
$$


onde

$$
[X]=\left[\begin{array}{ccccc}
-2 g Q & -2 g Q & 0 & 0 & -2 Q(F-D) \\
2 g Q & -2 g Q & -4 Q\left\langle\dot{e}_{0}, \dot{e}_{1}\right\rangle & 4 Q(G+L) & 2 Q(F-D) \\
-2 Q\left\langle\dot{e}_{0}, \dot{e}_{1}\right\rangle & -2 Q\left\langle\dot{e}_{0}, \dot{e}_{1}\right\rangle & 0 & 0 & Q(G+L) \\
4 Q\left\langle\dot{e}_{0}, \dot{e}_{1}\right\rangle & -4 Q\left\langle\dot{e}_{0}, \dot{e}_{1}\right\rangle & 2 g Q & 4 Q(F-D) & -2 Q(G+L) \\
4 Q\left\langle\dot{e}_{0}, \dot{e}_{1}\right\rangle & -4 Q\left\langle\dot{e}_{0}, \dot{e}_{1}\right\rangle & -2 g Q & 4 Q(D-F) & -2 Q(G+L)
\end{array}\right],
$$

$$
[Y]=\left[\begin{array}{l}
\dot{D} \\
\dot{F} \\
\dot{G}+\dot{L} \\
\left\langle\dot{e}_{0}, \dot{e}_{1}\right\rangle \\
\left|\dot{e}_{1}\right|^{2}
\end{array}\right]
$$

$\mathrm{e}$

$$
[W]=\left[\begin{array}{l}
(G-L)\left(4\left(F\left|\dot{e}_{1}\right|^{2}-D\right)+(D+F) g\right) \\
(G-L)\left((F-D) g+2\left\langle\dot{e}_{0}, \dot{e}_{1}\right\rangle(G+L)\right) \\
(G-L)\left(3\left\langle\dot{e}_{0}, \dot{e}_{1}\right\rangle(D+F)-\left(1+\left|\dot{e}_{1}\right|^{2}\right)(G+L)\right) \\
(G-L)\left(-2\left\langle\dot{e}_{0}, \dot{e}_{1}\right\rangle(D-F)-g(G+L)\right) \\
5(L-G)\left(2\left\langle\dot{e}_{0}, \dot{e}_{1}\right\rangle(D-F)-g(G+L)\right)
\end{array}\right]
$$

com

$$
g=\left(-1+\left|\dot{e}_{1}\right|^{2}\right), \quad \dot{Q}=L-G
$$

Após alguns cálculos, verifica-se que

$$
\operatorname{det}[X]=-256 Q^{5}\left\langle\dot{e}_{0}, \dot{e}_{1}\right\rangle\left(2\left\langle\dot{e}_{0}, \dot{e}_{1}\right\rangle(F-D)+g(G+L)\right)^{2},
$$

donde temos duas situações para analisar: quando a matriz $[X]$ é inversível e quando não é. Antes de analisarmos cada situação, observamos que por (2.25) segue-se que

$$
\begin{aligned}
\operatorname{det}[X]= & -256 Q^{5}\left\langle\dot{e}_{0}, \dot{e}_{1}\right\rangle\left(2\left\langle\dot{e}_{0}, \dot{e}_{1}\right\rangle(F-D)+g(G+L)\right)^{2} \\
= & -256 Q^{5}\left\langle\dot{e}_{0}, \dot{e}_{1}\right\rangle\left((G+L)\left(-1+\left|\dot{e}_{1}\right|^{2}\right)+2 L+\right. \\
& \left.-2\left|e_{1}\right|^{2} G-Q\left|\dot{e}_{1}\right|^{2}\right)^{2} \\
= & -256 Q^{5}\left\langle\dot{e}_{0}, \dot{e}_{1}\right\rangle\left(Q\left(1+\left|\dot{e}_{1}\right|^{2}\right)-Q\left|\dot{e}_{1}\right|^{2}\right)^{2} .
\end{aligned}
$$

Denotando por $[X]\left(s_{j}\right)$ a matriz $[X]$ aplicada no ponto $s_{j}$, temos que se existe um ponto $s_{1}$ em $\tilde{V}$ tal que $\operatorname{det}[X]\left(s_{1}\right) \neq 0$, então por continuidade, existe um intervalo 
$\tilde{U} \subset \tilde{V}$ contendo $s_{1}$ tal que $\operatorname{det}[X](s) \neq 0, \forall s \in \tilde{U}$. Nesta vizinhança é possível calcularmos explicitamente $[X]^{-1}$, dada por

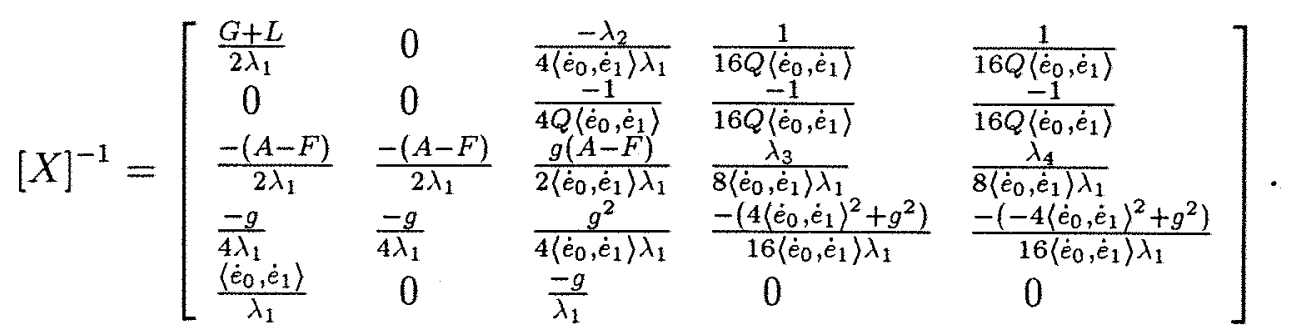

onde

$$
\begin{gathered}
\lambda_{1}(s)=Q\left(2\left\langle\dot{e}_{0}, \dot{e}_{1}\right\rangle(D-F)-g(G+L)\right), \\
\lambda_{2}(s)=2\left\langle\dot{e}_{0}, \dot{e}_{1}\right\rangle(D-F)+g(G+L), \\
\lambda_{3}(s)=(D-F) g-2(G+L)\left\langle\dot{e}_{0}, \dot{e}_{1}\right\rangle, \\
\lambda_{4}(s)=(D-F) g+2(G+L)\left\langle\dot{e}_{0}, \dot{e}_{1}\right\rangle .
\end{gathered}
$$

Da igualdade $[Y]=[X]^{-1} \cdot[W]$, obtém-se que

$$
\left\{\begin{aligned}
\dot{D} & =\frac{(L-G)\left(3 D\left\langle\dot{e}_{0}, \dot{e}_{1}\right\rangle+G+L\right)}{2 Q\left\langle\dot{e}_{0}, \dot{e}_{1}\right\rangle}, \\
\dot{F} & =\frac{-(L-G)\left(-3 F\left\langle\dot{e}_{0}, \dot{e}_{1}\right\rangle+G+L\right)}{2 Q\left\langle\dot{e}_{0}, \dot{e}_{1}\right\rangle} \\
(\dot{G}+\dot{L}) & =\frac{(L-G)\left(2(F-D)+3\left\langle\dot{e}_{0}, \dot{e}_{1}\right\rangle(G+L)\right)}{2 Q\left\langle\dot{e}_{0}, \dot{e}_{1}\right\rangle} \\
\left\langle\dot{e}_{0}, \dot{e}_{1}\right\rangle & =\frac{(L-G)\left(1+2\left\langle\dot{e}_{0}, \dot{e}_{1}\right\rangle^{2}-\left|\dot{e}_{1}\right|^{2}\right)}{2 Q\left\langle\dot{e}_{0}, \dot{e}_{1}\right\rangle} \\
\left|\dot{e}_{1}\right|^{2} & =\frac{(L-G)\left(1+\left|\dot{e}_{1}\right|^{2}\right)}{Q}
\end{aligned}\right.
$$

onde $Q=\sqrt{\left|\dot{e}_{1}\right|^{2}-\left\langle\dot{e}_{0}, \dot{e}_{1}\right\rangle^{2}}$.

Denotando-se por $U$ a vizinhança correspondente ao intervalo $\tilde{U}$ segue-se que, neste caso, para determinarmos quais são as condições que a superfície $M^{2}$ deve satisfazer na vizinhança $U$ para que a equação $K_{t} H_{s}-K_{s} H_{t}=0$ seja satisfeita basta, fixadas condições iniciais, resolver o sistema de equações diferenciais dado em (4.9). Observamos no entanto, que a última equação de (4.9) é equivalente ao $\operatorname{det}[X]=0$ em $U$, o que é uma contradição. Portanto $\operatorname{det}[X] \equiv 0$ em $\tilde{V}$. 
Denotando $f(s)=\left(2\left\langle\dot{e}_{0}, \dot{e}_{1}\right\rangle(F-D)+g(G+L)\right)$, temos que

$$
\operatorname{det}[X](s)=0 \Leftrightarrow\left\langle\dot{e}_{0}(s), \dot{e}_{1}(s)\right\rangle f^{2}(s)=0
$$

Suponhamos que existe um ponto $s_{2}$ em $\tilde{V}$ tal que $f\left(s_{2}\right) \neq 0$, então $\left\langle\dot{e}_{0}\left(s_{2}\right), \dot{e}_{1}\left(s_{2}\right)\right\rangle=$ 0. Como $(4.5)+(4.6)+(4.7)=0$, isto é,

$$
2\left\langle\dot{e}_{0}, \dot{e}_{1}\right\rangle Q \dot{F}+\left(-2\left\langle\dot{e}_{0}, \dot{e}_{1}\right\rangle F+G\right) \dot{Q}=0
$$

temos que no ponto $s_{2}$ vale que $G\left(s_{2}\right) \dot{Q}\left(s_{2}\right)=0$. Além disso, segue-se por (2.25) que $L\left(s_{2}\right)=0$, então usando-se o fato que $\dot{Q}=L-G$ segue-se que também $G\left(s_{2}\right)=0$, o que gera uma contradição, pois neste caso teríamos que $f\left(s_{2}\right)=0$. Logo,

$$
\operatorname{det}[X](s)=0 \Leftrightarrow f(s)=0,
$$

donde por (4.8) segue-se que na vizinhança $\tilde{V}$

$$
\begin{aligned}
\operatorname{det}[X]=0 & \Leftrightarrow\left(2\left\langle\dot{e}_{0}, \dot{e}_{1}\right\rangle(F-D)+g(G+L)\right)^{2}=0 \\
& \Leftrightarrow \dot{Q}\left(1+\left|\dot{e}_{1}\right|^{2}\right)-Q\left|\dot{e}_{1}\right|^{2}=0 \\
& \Leftrightarrow \frac{\left|\dot{e}_{1}\right|^{2}}{\left(1+\left|\dot{e}_{1}\right|^{2}\right)}=\frac{\dot{Q}}{Q} \\
& \Leftrightarrow \log \left(1+\left|\dot{e}_{1}\right|^{2}\right)=\log Q+\log \lambda \\
& \Leftrightarrow \frac{1+\left|\dot{e}_{1}\right|^{2}}{Q}=\lambda,
\end{aligned}
$$

onde $\lambda$ é uma constante real positiva não nula. Logo em uma vizinhança de cada ponto onde $K \neq 1$ vale que a função $F(s)$ é constante.

Consideremos o conjunto $A=\left\{p \in M^{2}: K(p) \neq 1\right\}$ e mostremos que $A=M^{2}$. Suponhamos que $A$ seja conexo. Se $A \varsubsetneqq M^{2}$, então existe pelo menos um ponto, $q$, no bordo de $A$ tal que $K(q)=1$, o que é uma contradição. De fato, como int $A \neq \emptyset$ existe uma seqüencia, de valor constante não nulo, tendendo para $q$, mas em $q$ temos que $Q\left(s_{i}\right)=0$, onde $q=\left(s_{i}, t_{i}\right)$, o que implica $F\left(s_{i}\right)=\infty$. Portanto $A=M^{2}$ e a função $F(s)$ é constante em $M^{2}$. Se $A$ não é conexo, chegamos a mesma contradição repetindo o raciocínio anterior em cada componente conexa de $A$.

Observamos que é imediato da última equivalência em (4.11) que $\left|\dot{e}_{1}\right|^{2}$ é constante se, e somente se, $\left\langle\dot{e}_{0}, \dot{e}_{1}\right\rangle$ for constante, mas estas funções não precisam ser constantes para que o quociente o seja.

Usando-se de toda a notação anterior temos que 
Teorema 4.2 Seja $M^{2} \subset \mathbb{S}^{3}$ uma superfície conexa, regrada não desenvolvível parametrizada como em (2.1). Suponha que se a função $F(s)$ for constante em $M^{2}$, então $Q(s)$ também é uma função constante em $M^{2}$. Sâo equivalentes:

i) $M^{2}$ é de Weingarten;

ii) as funçôes $\left|\dot{e}_{1}\right|^{2},\left\langle\dot{e}_{0}, \dot{e}_{1}\right\rangle, F, D, L$ e $G$ são constantes em $M^{2}$;

iii) $M^{2}$ é uma superfície helicoidal;

\section{Demonstração:}

i) $\Rightarrow$ ii)

Como $M^{2}$ é uma superfície de Weingarten segue-se, pelo Lema (4.1), que a função $\digamma(s)$ é constante em $M^{2}$ e portanto por hipótese as funções $\left|\dot{e}_{1}\right|^{2}$ e $\left\langle\dot{e}_{0}, \dot{e}_{1}\right\rangle^{2}$ são funções constantes distintas em $M^{2}$.

Temos então por (2.11) que $K_{s} \equiv 0 \mathrm{em} M^{2}$ e portanto pela Proposição (1.22), $H_{s} \equiv 0 \mathrm{em} M^{2}$. Mas como $\left|X_{s}\right|^{2}$ é constante em relação a $s, H_{s} \equiv 0$ se, e somente se, $h_{s} \equiv 0$, ou equivalentemente, se as funções $F, D, G$ e $L$ são constantes em $M^{2}$.

A demonstração que (ii) implica (i) é imediata. Os outros casos seguem diretamente do teorema (3.3).

\subsection{Classificação das superfícies regradas e de Wein- garten em $\mathbf{H}^{3}$}

Como já mencionado, nesta seção estaremos classificando as superfícies regradas de Weingarten em $\mathbb{H}^{3}$. Apesar do raciocínio usado ser exatamente o mesmo da seção anterior, não unimos as duas seções, como já feito em outros casos, pois existe a troca de sinal em vários lugares. Por este motivo, para não tornarmos a leitura repetitiva iremos apenas escrever o fundamental.

Lema 4.3 Seja $M^{2} \subset \mathbb{H}^{3}$ uma superficie conexa regrada não desenvolvível e parametrizada como em (2.1). Se a equação $K_{t} H_{s}-K_{s} H_{t}=0$ é satisfeita, então a função $\digamma(s)=\frac{-1+\left|\dot{e}_{1}\right|^{2}}{Q} e ́$ constante em cada componente conexa onde $K \neq-1$.

\section{Demonstração:}

Como $M^{2}$ é uma superfície não desenvolvível, existe um ponto $p \in M^{2}$ tal que $K(p) \neq-1$, o que é equivalente a dizer que $Q\left(s_{0}\right) \neq 0$, onde $p=\left(s_{0}, t_{0}\right)$. Logo existe 
um intervalo $\tilde{V} \subset \mathbb{R}$ contendo $s_{0}$ tal que $Q(s) \neq 0 \forall s \in \tilde{V}$, ou equivalentemente, existe uma vizinhança $V \subset M^{2}$ de $p$ tal que $K(q) \neq-1, \forall q \in V$.

Não é difícil mostrar, que na vizinhança $V$ vale que

$$
\begin{aligned}
H_{s} K_{t}-H_{t} K_{s}=0 & \Leftrightarrow-h_{t}\left|X_{s}\right|^{2}\left(\dot{Q}\left|X_{s}\right|^{2}-2 Q\left\langle X_{s s}, X_{s}\right\rangle\right)+ \\
& -2 Q h_{s}\left|X_{s}\right|^{2}\left\langle X_{s t}, X_{s}\right\rangle+3 h \dot{Q}\left|X_{s}\right|^{2}\left\langle X_{s t}, X_{s}\right\rangle=0,
\end{aligned}
$$

ou equivalentemente,

$$
-h_{t}\left|X_{s}\right|^{2}\left(\frac{Q}{\left|X_{s}\right|^{2}}\right)_{s}+h \dot{Q}\left\langle X_{s t}, X_{s}\right\rangle+\left(\left|X_{s}\right|^{2}\right)_{t} h^{2}\left(\frac{Q}{h}\right)_{s}=0
$$

É natural que aqui valha o mesmo comentário feito sobre a equação (4.2), isto é, o estudo da equação anterior mostrou-se muito complicado devido a forma como ela esta apresentada. Fazendo-se as alterações necessárias, mostra-se após vários cálculos, que na vizinhança $V$ vale a equação

$$
\begin{aligned}
H_{s} K_{t}-H_{t} K_{s}=0 & \Leftrightarrow \frac{1}{2} Q\left|X_{s}\right|^{2} \mathcal{J}(s, t)=0 \\
& \Leftrightarrow \mathcal{J}(s, t)=0,
\end{aligned}
$$

onde

$$
\mathcal{J}(s, t)=g_{1}(s) \sinh 2 t+g_{2}(s) \sinh 4 t+g_{3}(s) \cosh 2 t+g_{4}(s) \cosh 4 t+g_{5}(s),
$$

com

$$
\begin{aligned}
g_{1}(s) & =2 Q\left(-\left(1+\left|\dot{e}_{1}\right|^{2}\right) \dot{D}+(F+D)\left|\dot{e}_{1}\right|^{2}+\left(1+\left|\dot{e}_{1}\right|^{2}\right) \dot{F}\right)+ \\
& +\left(D\left(5+\left|\dot{e}_{1}\right|^{2}\right)-\left(1+5\left|\dot{e}_{1}\right|^{2}\right) F\right) \dot{Q} \\
g_{2}(s) & =2 Q\left(\left(1+\left|\dot{e}_{1}\right|^{2}\right) \dot{D}-2(G+L)\left\langle\dot{e}_{0}, \dot{e}_{1}\right\rangle-(F+D)\left|\dot{e}_{1}\right|^{2}+\right. \\
& \left.+\left(1+\left|\dot{e}_{1}\right|^{2}\right) \dot{F}+2\left\langle\dot{e}_{0}, \dot{e}_{1}\right\rangle(\dot{G}+\dot{L})\right)+ \\
& -\left(D\left(1+\left|\dot{e}_{1}\right|^{2}\right)+\left(1+\left|\dot{e}_{1}\right|^{2}\right) F+\right. \\
& \left.+2\left\langle\dot{e}_{0}, \dot{e}_{1}\right\rangle(G+L)\right) \dot{Q} \\
& \\
g_{3}(s)= & (G+L)\left(Q\left|\dot{e}_{1}\right|^{2}-\left(-1+\left|\dot{e}_{1}\right|^{2}\right) \dot{Q}\right)+\left\langle\dot{e}_{0}, \dot{e}_{1}\right\rangle(2 Q(-\dot{D}+\dot{F})+ \\
+ & 3(D-F) \dot{Q})
\end{aligned}
$$




$$
\begin{aligned}
g_{4}(s) & =2 Q\left(-2(F+D)\left\langle\dot{e}_{0}, \dot{e}_{1}\right\rangle-(G+L)\left|\dot{e}_{1}\right|^{2}+2\left\langle\dot{e}_{0}, \dot{e}_{1}\right\rangle(\dot{D}+\right. \\
& \left.+\dot{F})+\left(1+\left|\dot{e}_{1}\right|^{2}\right)(\dot{G}+\dot{L})\right)-\left(2\left\langle\dot{e}_{0}, \dot{e}_{1}\right\rangle(D+\right. \\
& \left.+F)+\left(1+\left|\dot{e}_{1}\right|^{2}\right)(G+L)\right) \dot{Q}, \\
g_{5}(s) & =2 Q\left(2(D+F)\left\langle\dot{e}_{0}, \dot{e}_{1}\right\rangle-(G+L)\left|\dot{e}_{1}\right|^{2}+2\left\langle\dot{e}_{0}, \dot{e}_{1}\right\rangle(\dot{D}+\right. \\
& \left.+\dot{F})-\left(1+\left|\dot{e}_{1}\right|^{2}\right)(\dot{G}+\dot{L})\right)+5\left(-2\left\langle\dot{e}_{0}, \dot{e}_{1}\right\rangle(D+\right. \\
& \left.+F)+\left(1+\left|\dot{e}_{1}\right|^{2}\right)(G+L)\right) \dot{Q} .
\end{aligned}
$$

Analogamente ao caso esférico, temos que na vizinhança de um ponto onde $K \neq-1$ a equação $K_{t} H_{s}-K_{s} H_{t}=0$ é satisfeita se, e somente se, $g_{1}(s)=g_{2}(s)=g_{3}(s)=$ $g_{4}(s)=g_{5}(s)=0$. Passemos então ao estudo destas cinco EDO's.

É fácil ver que as EDO's anteriores verificam a seguinte relação matricial

$$
[X]_{5 \times 5} \cdot[Y]_{5 \times 1}=[W]_{5 \times 1}
$$

onde

$$
\begin{gathered}
{[X]=\left[\begin{array}{ccccc}
-2 g Q & 2 g Q & 0 & 0 & 2 Q(F+D) \\
2 g Q & 2 g Q & 4 Q\left\langle\dot{e}_{0}, \dot{e}_{1}\right\rangle & -4 Q(G+L) & -2 Q(F+D) \\
-2 Q\left\langle\dot{e}_{0}, \dot{e}_{1}\right\rangle & 2 Q\left\langle\dot{e}_{0}, \dot{e}_{1}\right\rangle & 0 & 0 & Q(G+L) \\
4 Q\left\langle\dot{e}_{0}, \dot{e}_{1}\right\rangle & 4 Q\left\langle\dot{e}_{0}, \dot{e}_{1}\right\rangle & 2 g Q & -4 Q(F+D) & -2 Q(G+L) \\
4 Q\left\langle\dot{e}_{0}, \dot{e}_{1}\right\rangle & 4 Q\left\langle\dot{e}_{0}, \dot{e}_{1}\right\rangle & -2 g Q & 4 Q(D+F) & -2 Q(G+L)
\end{array}\right]} \\
{[Y]=\left[\begin{array}{c}
\dot{D} \\
\dot{F} \\
\dot{G}+\dot{L} \\
\left\langle\dot{e}_{0}, \dot{e}_{1}\right\rangle \\
\left.\dot{e}_{1}\right|^{2}
\end{array}\right]}
\end{gathered}
$$

e

$$
[W]=\left[\begin{array}{l}
-(G-L)\left(4\left(-F\left|\dot{e}_{1}\right|^{2}+D\right)+(D-F) g\right) \\
(G-L)\left((F+D) g+2\left\langle\dot{e}_{0}, \dot{e}_{1}\right\rangle(G+L)\right) \\
-(G-L)\left(3\left\langle\dot{e}_{0}, \dot{e}_{1}\right\rangle(D-F)-\left(-1+\left|\dot{e}_{1}\right|^{2}\right)(G+L)\right) \\
(G-L)\left(2\left\langle\dot{e}_{0}, \dot{e}_{1}\right\rangle(D+F)+g(G+L)\right) \\
5(L-G)\left(-2\left\langle\dot{e}_{0}, \dot{e}_{1}\right\rangle(D+F)+g(G+L)\right)
\end{array}\right]
$$


com

$$
g=\left(1+\left|\dot{e}_{1}\right|^{2}\right), \quad \dot{Q}=G-L
$$

Após alguns cálculos, verifica-se que

$$
\operatorname{det}[X]=-256 Q^{5}\left\langle\dot{e}_{0}, \dot{e}_{1}\right\rangle\left(2\left\langle\dot{e}_{0}, \dot{e}_{1}\right\rangle(F+D)-g(G+L)\right)^{2}
$$

donde temos novamente duas situações para analisar: quando a matriz [X] é inversível e quando não é. Observamos que por (2.25) segue-se que

$$
\begin{aligned}
\operatorname{det}[X]= & -256 Q^{5}\left\langle\dot{e}_{0}, \dot{e}_{1}\right\rangle\left(2\left\langle\dot{e}_{0}, \dot{e}_{1}\right\rangle(F+D)-g(G+L)\right)^{2} \\
= & -256 Q^{5}\left\langle\dot{e}_{0}, \dot{e}_{1}\right\rangle\left(-(G+L)\left(-1+\left|\dot{e}_{1}\right|^{2}\right)+2 L+\right. \\
& \left.+2\left|\dot{e}_{1}\right|^{2} G-Q\left|\dot{e}_{1}\right|^{2}\right)^{2} \\
= & -256 Q^{5}\left\langle\dot{e}_{0}, \dot{e}_{1}\right\rangle\left(\dot{Q}\left(-1+\left|\dot{e}_{1}\right|^{2}\right)-Q\left|\dot{e}_{1}\right|^{2}\right)^{2} .
\end{aligned}
$$

Se existe um ponto $s_{1}$ em $\tilde{V}$ tal que $\operatorname{det}[X]\left(s_{1}\right) \neq 0$, então por continuidade, existe um intervalo $\tilde{U} \subset \tilde{V}$ contendo $s_{1}$ tal que $\operatorname{det}[X](s) \neq 0, \forall s \in \tilde{U}$. Nesta vizinhança é possivel calcular explicitamente $[X]^{-1}$, isto é,

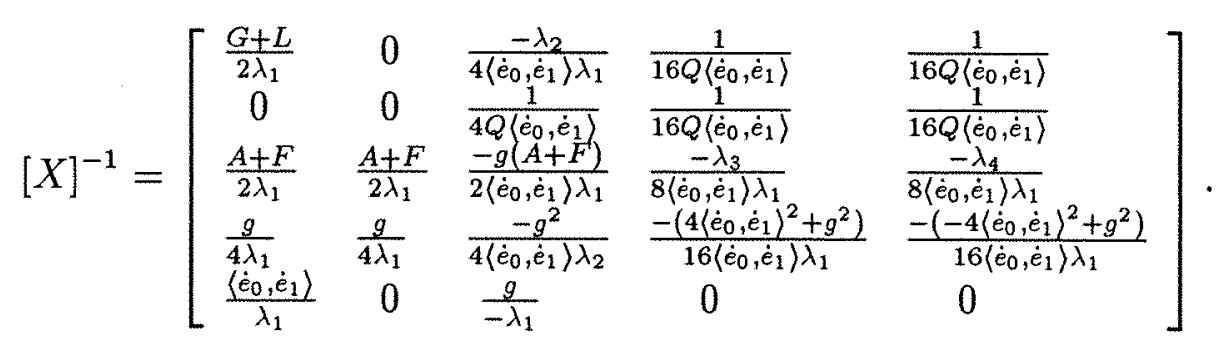

onde

$$
\begin{gathered}
\lambda_{1}(s)=Q\left(2\left\langle\dot{e}_{0}, \dot{e}_{1}\right\rangle(D+F)-g(G+L)\right), \\
\lambda_{2}(s)=2\left\langle\dot{e}_{0}, \dot{e}_{1}\right\rangle(D+F)+g(G+L), \\
\lambda_{3}(s)=(D+F) g+2(G+L)\left\langle\dot{e}_{0}, \dot{e}_{1}\right\rangle, \\
\lambda_{4}(s)=(D+F) g-2(G+L)\left\langle\dot{e}_{0}, \dot{e}_{1}\right\rangle .
\end{gathered}
$$


Da igualdade $[Y]=[X]^{-1} \cdot[W]$, obtém-se que

$$
\left\{\begin{array}{c}
\dot{D}=\frac{-(L-G)\left(3 D\left\langle\dot{e}_{0}, \dot{e}_{1}\right\rangle-G-L\right)}{2 Q\left\langle\dot{e}_{0}, \dot{e}_{1}\right\rangle} \\
\dot{F}=\frac{-(L-G)\left(6 F\left\langle\dot{e}_{0}, \dot{e}_{1}\right\rangle-2(G+L)\right)}{2 Q\left\langle\dot{e}_{0}, \dot{e}_{1}\right\rangle} \\
(\dot{G}+\dot{L})=\frac{-(L-G)\left(-2(F+D)+3\left\langle\dot{e}_{0}, \dot{e}_{1}\right\rangle(G+L)\right)}{2 Q\left\langle\dot{e}_{0}, \dot{e}_{1}\right\rangle} \\
\left\langle\dot{e}_{0}, \dot{e}_{1}\right\rangle=\frac{-(L-G)\left(-1+2\left\langle\dot{e}_{0}, \dot{e}_{1}\right\rangle^{2}-\left|\dot{e}_{1}\right|^{2}\right)}{2 Q\left\langle\dot{e}_{0}, \dot{e}_{1}\right\rangle} \\
\left|\dot{e}_{1}\right|^{2}=\frac{-(L-G)\left(-1+\left|\dot{e}_{1}\right|^{2}\right)}{Q}
\end{array}\right.
$$

onde $Q=\sqrt{\left|\dot{e}_{1}\right|^{2}-\left\langle\dot{e}_{0}, \dot{e}_{1}\right\rangle^{2}}$.

Denotando-se por $U$ a vizinhança correspondente ao intervalo $\tilde{U}$, segue-se analogamente ao caso esférico, que para determinarmos quais são as condições que a superfície $M^{2}$ deve satisfazer na vizinhança $U$ para que a equação $K_{t} H_{s}-K_{s} H_{t}=0$ seja satisfeita basta, fixadas condições iniciais, resolver o sistema de equações diferenciais em (4.18). Observamos no entanto, que a última equação de (4.18) é equivalente ao $\operatorname{det}[X]=0$ em $U$, o que é uma contradição. Portanto $\operatorname{det}[X] \equiv 0$ em $\tilde{V}$.

Denotando agora $f(s)=\left(2\left\langle\dot{e}_{0}, \dot{e}_{1}\right\rangle(F+D)-g(G+L)\right)$, temos que

$$
\operatorname{det}[X](s)=0 \Leftrightarrow\left\langle\dot{e}_{0}(s), \dot{e}_{1}(s)\right\rangle f^{2}(s)=0 .
$$

Se supormos, analogamente ao caso esférico, que existe um ponto $s_{2}$ em $\tilde{V}$ tal que $f\left(s_{2}\right) \neq 0$, chegaremos novamente em uma contradição Logo,

$$
\operatorname{det}[X](s)=0 \Leftrightarrow f(s)=0,
$$

donde segue-se por (4.17) que

$$
\operatorname{det}[X]=0 \Leftrightarrow \dot{Q}\left(-1+\left|\dot{e}_{1}\right|^{2}\right)-Q\left|\dot{e}_{1}\right|^{2}=0 .
$$

$\operatorname{Se}\left(-1+\left|\dot{e}_{1}\right|^{2}\right)>0$, então

$$
\begin{aligned}
\operatorname{det}[X]=0 & \Leftrightarrow \frac{\left|\dot{e}_{1}\right|^{2}}{\left(-1+\left|\dot{e}_{1}\right|^{2}\right)}=\frac{\dot{Q}}{Q} \\
& \Leftrightarrow \log \left(-1+\left|\dot{e}_{1}\right|^{2}\right)=\log Q+\log \lambda_{1} \\
& \Leftrightarrow \frac{-1+\left|\dot{e}_{1}\right|^{2}}{Q}=\lambda_{1},
\end{aligned}
$$


onde $\lambda_{1}$ é uma constante real positiva não nula. Se $\left(-1+\left|\dot{e}_{1}\right|^{2}\right)<0$, então

$$
\begin{aligned}
\operatorname{det}[X]=0 & \Leftrightarrow-\dot{Q}\left(1-\left|\dot{e}_{1}\right|^{2}\right)-Q\left|\dot{e}_{1}\right|^{2}=0 \\
& \Leftrightarrow \frac{-\left|\dot{e}^{2}\right|^{2}}{\left(1-\left|\dot{e}_{1}\right|^{2}\right)}=\frac{\dot{Q}}{Q} \\
& \Leftrightarrow \log \left(1-\left|\dot{e}_{1}\right|^{2}\right)=\log Q+\log \lambda_{2} \\
& \Leftrightarrow \frac{1-\left|\dot{e}_{1}\right|^{2}}{Q}=\lambda_{2},
\end{aligned}
$$

onde $\lambda_{2}$ é uma constante real positiva não nula.

Logo em uma vizinhança de cada ponto onde $K \neq-1$, vale o desejado. Observe que $F(s)=0$ quando $\left|\dot{e}_{1}\right|^{2}=1$.

Usando-se de toda a notação anterior, segue-se no caso hiperbólico um teorema análogo ao Teorema (4.2):

Teorema 4.4 Seja $M^{2} \subset \mathbb{H}^{3}$ uma superfície regrada não desenvolvivel parametrizada como em (2.1). Suponha ainda que se a função $F(s)$ for localmente constante, então $Q(s)$ também é uma função localmente constante. São equivalentes:

i) $M^{2}$ é de Weingarten;

ii) as funções $\left|\dot{e}_{1}\right|^{2},\left\langle\dot{e}_{0}, \dot{e}_{1}\right\rangle, F, D, L$ e $G$ são constantes em $M^{2}$;

iii) $M^{2}$ é uma superfície helicoidal;

\section{Demonstração:}

Consideremos o conjunto aberto $A=\left\{p \in M^{2}: K(p) \neq-1\right\}$. Como $M^{2}$ é uma superfície de Weingarten temos pelo Lema (4.3) que a função $\digamma(s)$ é constante em cada componente conexa de $A$, e portanto por hipótese as funções $\left|\dot{e}_{1}\right|^{2}$ e $\left\langle\dot{e}_{0}, \dot{e}_{1}\right\rangle^{2}$ são funções constantes distintas em cada componente conexa de $A$.

Segue-se, ainda por (2.11), que em cada componente conexa de $A, K_{s} \equiv 0$ e portanto pela Proposição (1.22), $H_{s} \equiv 0$ em cada componente. Mas como $\left|X_{s}\right|^{2}$ é constante em relação a $s, H_{s} \equiv 0$ se, e somente se, $h_{s} \equiv 0$, ou equivalentemente, se as funções $F, D, G$ e $L$ são constantes em cada componente conexa de $A$.

Mostremos que $A=M^{2}$. Suponhamos que $A$ seja conexo. Se $A \nsubseteq M^{2}$, então existe pelo menos um ponto no bordo de $A$ onde a função $Q$ se anula, o que é um absurdo, pois como int $A \neq \emptyset$ existiria uma seqüencia, de valor constante não nulo, tendendo para zero. Portanto $A=M^{2}$. Se $A$ não é conexo, chegamos a mesma contradição repetindo o raciocínio anterior em cada componente conexa de $A$.

Logo se $M^{2}$ é uma superfície regrada não desenvolvível e de Weingarten, então $Q$ é função constante não nula em $M^{2}$. Observe, no entanto, que $Q=0$ se, e somente 
se, $K=-1$, portanto se existe um ponto onde $K \neq-1$, então $K$ nunca assumirá o valor -1 em $M^{2}$.

Segue-se ainda que $F, D, G$ e $L$ são funções constantes em $M^{2}$, pois $M^{2}$ é conexo e estas funções são constantes em $A$.

A demonstração que (ii) implica ( $i$ ) é imediata. Os outros casos seguem diretamente do teorema (3.3).

Antes de terminarmos este capítulo, gostaríamos de dizer que o ideal seria ter conseguido demonstrar os teoremas (4.2) e (4.4) sem a hipótese adicional de que se a função $F(s)$ for localmente constante, então a função $Q(s)$ também é constante.

Vários cálculos foram feitos com o intuito de se entender qual seria o comportamento de uma superfície regrada em $\mathbb{Q}^{3}(c), c \neq 0$, que satisfaz a condição de que a função $\digamma(s)$ é localmente constante mas a função $Q(s)$ não é localmente constante, no entanto quase nada conseguimos. Não conseguimos garantir, por exemplo, nem que as funções $g_{j}{ }^{6} s(s)$ dadas em (4.3)-(4.7), no caso esférico, e (4.12)-(4.16), no caso hiperbólico, são nulas.

Como não conseguimos encontrar exemplos de superfícies regradas em $\mathbb{Q}^{3}(c), c \neq$ 0 , que satisfaçam esta condição, nem contra exemplos, esperamos que ainda possam ser encontrados resultados nesta direção. 


\section{Capítulo 5}

\section{Hipersuperfícies regradas e de Weingarten em formas espaciais}

Neste capítulo estenderemos a classificação feita no capítulo anterior para o caso de hipersuperfícies, ou seja, classificaremos as hipersuperfícies regradas e de Weingarten em $\mathbb{S}^{n+1}$ e $\mathbb{H}^{n+1}, n \geq 3$. O caso euclidiano foi resolvido por Dajczer e Tenenblat em [DT]. Para classificarmos tais hipersuperfícies é fundamental alguns resultados descritos por Dajczer e Gromoll [DG] que parametrizam, pelo menos localmente, as hipersuperfícies em formas espaciais com índice de nulidade relativa constante. $\mathrm{Na}$ seção 5.1 daremos algumas definições básicas e discutiremos os resultados descritos por Dajczer e Gromoll. Nas seções 5.2 e 5.3 discutiremos a classificação das hipersuperfícies regradas e de Weingarten em $\mathbb{S}^{n+1}$ e $\mathbb{H}^{n+1}$, respectivamente.

\subsection{Parametrização de Gauss de uma hipersuperfície em uma forma espacial}

A definição usual de aplicação de Gauss em $\mathbb{R}^{n+1}$ estende-se naturalmente para formas espaciais, isto é, seja $f: M^{n} \rightarrow \mathbb{Q}^{n+1}(c)$ uma imersão isométrica de uma variedade Riemanniana conexa orientável em $\mathbb{Q}^{n+1}(c)$. Então o campo unitário normal a $M^{n}$ 
em $\mathbb{Q}^{n+1}(c)$ induz uma aplicação

$$
\begin{aligned}
\eta: & M^{n} \rightarrow \mathbb{S}^{n}, \quad \text { se } \mathrm{c}=0 \\
& x \longrightarrow \eta_{x} \\
\eta: & M^{n} \rightarrow \mathbb{S}^{n+1}, \quad \text { se } \mathrm{c}=1 \\
& x \longrightarrow \eta_{x} \\
\eta: & M^{n} \rightarrow \mathbb{S}_{1}^{n+1}, \quad \text { se } \mathrm{c}=-1 \\
& x \longrightarrow \eta_{x}
\end{aligned}
$$

chamada apenas de aplicação de Gauss se $c=0$, e de aplicação de Gauss esférica ou hiperbólica se $c=1$ ou $c=-1$, respectivamente. Geometricamente, nos três casos, a aplicação $\eta$ associa a cada ponto $x \in M^{n}$ o ponto $\eta_{x}$, obtido fazendo-se o transporte paralelo do vetor $\eta_{x} \in T_{x} M^{\perp}$ até a origem de $\mathbb{R}^{n+1}$, se $c=0$, de $\mathbb{R}^{n+2}$, se $c=1$, ou de $\mathbb{L}^{n+2}$, se $c=-1$.

Em geral, a aplicação de Gauss em $\mathbb{Q}^{n+1}(c)$ não é inversível, no entanto, quando colocamos uma hipótese adicional sobre a hipersuperfície (índice de nulidade relativa constante) tal inversão é possível. No que se segue, parametrizaremos uma hipersuperfície em $\mathbb{Q}^{n+1}(c)$, pelo menos localmente, usando-se a inversa da aplicação de Gauss (no fibrado normal à sua imagem), que será chamada de parametrização de Gauss.

Sejam agora $f: M^{n} \rightarrow \mathbb{Q}^{n+1}(c)$ uma imersão isométrica de uma variedade Riemanniana conexa em $\mathbb{Q}^{n+1}(c)$ e $\alpha$ a sua segunda forma fundamental.

Denotando-se por $x$ um ponto em $M^{n}$, temos que o subespaço de $T_{x} M$ dado por

$$
\Delta(x)=\left\{X \in T_{x} M: \alpha(X, Y)=0, \quad \forall Y \in T_{x} M\right\}
$$

é chamado de subespaço de nulidade relativa de $f$ em $x$. Note que $\Delta(x)=\operatorname{Ker} \alpha$. Seja ainda, $\nu(x)=\operatorname{dim} \Delta(x)$ o indice de nulidade relativa de $f$ em $x$ e $\nu_{0}$ o mínimo do índice de nulidade relativa de $f$, definido por $0 \leq \nu_{0}=\min _{x \in M} \nu(x) \leq n$.

É bem conhecido ( veja-se [D] e [F] ) que o conjunto $M_{0}=\left\{x \in M: \nu(x)=\nu_{0}\right\}$ é não vazio e aberto em $M, \Delta$ é uma distribuição diferenciável e involutiva em $M_{0}$ e cada folha da distribuição é totalmente geodésica em $M^{n}$ e em $\mathbb{Q}^{n+1}(c)$.

Suponhamos, no que se segue, que a imersão $f$ tem índice de nulidade relativa constante, isto é, $M=M_{0} \operatorname{com} \nu_{0}=n-k$. Tomemos $U$ um subconjunto aberto de $M$ e seja $\pi: U \rightarrow V$ a projeção de $U$ em $V$, onde $V$ é o espaço quociente das folhas da distribuição em $U$. Segue-se direto do fato que $\nu(x)=n-k, \forall x \in M$, que $V$ é uma variedade diferenciável de dimensão $k$. Toda vez que falarmos sobre as "folhas", estaremos nos referindo as folhas da distribuição da nulidade relativa. 
Seja $\eta$ o campo normal unitário definido sobre o aberto $U$. Observe que, a menos de sinal, $\eta$ é único. É imediato mostrar que $\eta$ é paralelo ao longo das folhas, na conexão normal. De fato

$$
\left(\bar{\nabla}_{X} \eta\right)^{T}=-A_{\eta} X=0
$$

para todo $X \in \Delta(x)$ e todo $x \in U$, onde $\bar{\nabla}$ é a conexão de Levi-Civita de $\mathbb{Q}^{n+1}(c)$.

Utilizando-se estes conceitos é possível caracterizar localmente as hipersuperfícies em formas espaciais com índice de nulidade relativa constante. Os próximos resultados e suas respectivas demonstrações encontram-se em [DG]. Observamos apenas que a aplicação de Gauss, $\eta$, induz uma imersão $g \operatorname{de~} V$ em $\mathbb{S}^{n}$, se $c=0$, em $\mathbb{S}^{n+1}$, se $c=1$ ou em $\mathbb{S}_{1}^{n+1}$, se $c=-1$, tal que $\eta=g \circ \pi$.

Teorema 5.1 Sejam $g: V^{k} \rightarrow \mathbb{S}^{n}$ uma imersão isométrica e $\gamma$ uma função em $V^{k}$. Considere a aplicação $\psi: \Lambda \rightarrow \mathbb{R}^{n+1}$ definida no fibrado normal, $\Lambda$, ao longo da imersão $g$, dada por

$$
\psi(y, w)=\gamma g+\nabla \gamma+w
$$

Então, no subconjunto aberto de pontos regulares, $\psi$ determina uma hipersuperfície imersa com indice de nulidade relativa constante $n-k$. Reciprocamente, qualquer hipersuperfície de $\mathbb{R}^{n+1}$ com indice de nulidade relativa constante pode ser obtida desta forma, pelo menos localmente.

Proposição 5.2 Com a notação do teorema anterior, temos:

(i) $\psi$ tem posto máximo $n$ em $(y, w)$ se, e somente se, o operador autoadjunto

$$
P=\gamma(y) I+H_{\gamma(y)}-B_{w}
$$

é não singular no espaço tangente a $V^{k}$ em $y$, onde $H_{\gamma}$ é o hessiano em $V^{k}$ e $B_{w} e ́$ a matriz da segunda forma fundamental de $g$ em y relativa a $w$.

(ii) Em tais pontos, $g$ é o campo normal unitário à imersão $\psi$, a matriz da segunda forma fundamental, $A=A_{g}$, em $\mathbb{R}^{n+1}$ tem posto $k e$

$$
A=-P^{-1} \quad \text { em } \Delta^{\perp}
$$

onde $\Delta^{\perp}$ é o complemento ortogonal do subespaço de nulidade relativa.

Corolário 5.3 Seja $g: V^{k} \rightarrow \mathbb{S}^{n+1}$ uma imersão isométrica. Considere a aplicação $\psi: \Lambda^{1} \longrightarrow \mathbb{S}^{n+1}$ definida no fibrado normal unitário, $\Lambda^{1}$, ao longo da imersão $\mathrm{g}$, dada por

$$
\psi(y, w)=w
$$




\section{Então:}

(i) No subconjunto aberto de pontos regulares, $\psi$ determina uma hipersuperficie imersa com indice de nulidade relativa constante $n-k$.

(ii) Reciprocamente, qualquer hipersuperficie de $\mathbb{S}^{n+1}$ com indice de nulidade relativa constante pode ser obtida desta forma, pelo menos localmente.

(iii) $\psi$ possui posto máximo $n$ no ponto $(y, w)$ se, e somente se, a matriz da segunda forma fundamental da imersão $g$ na direção $w$ é não-singular. Em tais pontos, $g$ é vetor normal unitário à imersão $\psi$, a matriz da segunda forma fundamental, $A$, da imersão $\psi$ em $\mathbb{S}^{n+1}$ tem posto $k e$

$$
A(y, w)=A_{g}(y, w)=B_{w}^{-1}(y) \text { em } \Delta^{\perp},
$$

onde $\Delta^{\perp}$ é o complemento ortogonal da nulidade relativa e $B$ é a segunda forma fundamental da imersão $g$.

O caso hiperbólico pode ser estudado de forma análoga ao caso esférico, isto é, seja $g: V^{k} \rightarrow \mathbb{S}_{1}^{n+1} \subset \mathbb{L}^{n+2}$ uma imersão isométrica de uma variedade Riemanniana no espaço de De Sitter. Considerando o fibrado normal unitário, $\Lambda^{1}$, ao longo da imersão $g$, isto é, o conjunto de pares $(y, w)$, onde $y \in V^{k}$ e $w$ é um vetor normal a $V^{k}$ em $y$ de comprimento $\left.-1(\langle w, w\rangle)=-1\right)$, então $\psi: \Lambda^{1} \rightarrow \mathbb{H}^{n+1} \subset \mathbb{L}^{n+2}$ dada por $\psi(y, w)=w$, determina, nos pontos regulares, uma hipersuperfície imersa com índice de nulidade relativa constante $\nu=n-k$. Segue-se então, que podemos enunciar para o caso hiperbólico um corolário análogo ao anterior, melhor dizendo: Seja $g: V^{k} \rightarrow \mathbb{S}_{1}^{n+1}$ uma imersão isométrica tipo espaço. Considere a aplicação $\psi: \Lambda^{1} \longrightarrow \mathbb{H}_{1}^{n+1}$ definida no fibrado normal unitário, $\Lambda^{1}$, ao longo da imersão $g$, dada por

$$
\psi(y, w)=w
$$

Então:

(i) No subconjunto aberto de pontos regulares, $\psi$ determina uma hipersuperfície imersa com índice de nulidade relativa constante $n-k$;

(ii) Reciprocamente, qualquer hipersuperfície de $\mathbb{H}_{1}^{n+1}$ com índice de nulidade relativa constante pode ser obtida desta forma, pelo menos localmente;

(iii) $\psi$ possui posto máximo $n$ no ponto $(y, w)$ se, e somente se, a matriz da segunda forma fundamental da imersão $g$ na direção $w$ é não-singular. Em tais pontos, $g$ é vetor normal unitário à imersão $\psi$, a matriz da segunda forma fundamental, $A$, da imersão $\psi$ em $\mathbb{H}_{1}^{n+1}$ tem posto $k$ e

$$
A(y, w)=A_{g}(y, w)=B_{w}^{-1}(y) \quad \text { em } \quad \Delta^{\perp}
$$


onde $\Delta^{\perp}$ é o complemento ortogonal da nulidade relativa e $B$ é a segunda forma fundamental da imersão $g$.

Antes de terminarmos esta seção, faremos algumas observações que serão usadas nas próximas seções.

Observação 5.4 Sejam $M^{n}, n \geq 3$, uma hipersuperfície conexa regrada em $\mathbb{Q}^{n+1}(c)$ e $\bar{M}=\left\{p \in M^{n}: S(p) \neq c\right\}$, onde $S$ é a curvatura escalar de $M$. Como $M$ é uma hipersuperfície regrada, segue-se que a curvatura seccional em pontos de $\bar{M}$, em relação a algum subespaço bi-dimensional do espaço tangente a $M^{n}$, não é igual a $c$. Observe que para qualquer hipersuperfície vale a afirmação anterior, mas sendo $M^{n}$ regrada temos que estes dois conjuntos coincidem, isto é, em todo ponto onde existe um subespaço bi-dimensional do espaço tangente a $M^{n}$ tal que a curvatura seccional não é igual a $c$, a curvatura escalar é diferente de $c$. De fato, seja $\left\{Y_{1}, Y_{2}, \ldots . Y_{n}\right\}$ uma base ortonormal para o espaço tangente a $M^{n}$ em $p$, tal que $Y_{j}, 1 \leq j \leq n-1$, são tangentes à folha que regra a hipersuperfície. Lembrando-se que $\alpha\left(Y_{j}, Y_{i}\right)=0$, $1 \leq i, j \leq n-1$, onde $\alpha$ é a segunda forma fundamental da imersão, segue-se pela equação de Gauss que

$$
\begin{gathered}
K\left(Y_{i}, Y_{j}\right)=c+\left\langle\alpha\left(Y_{i}, Y_{i}\right), \alpha\left(Y_{j}, Y_{j}\right)\right\rangle-\left|\alpha\left(Y_{i}, Y_{j}\right)\right|^{2} \\
=c, \quad 1 \leq i, j \leq n-1 \\
\quad e \\
K\left(Y_{k}, Y_{n}\right)=c-\left|\alpha\left(Y_{k}, Y_{n}\right)\right|^{2}, \quad 1 \leq k \leq n-1 .
\end{gathered}
$$

Portanto se $p$ é um ponto em $M^{n}$ onde a curvatura seccional é menor do que $c$, então $S(p) \neq c$, pois

$$
\begin{aligned}
S & =\frac{1}{n(n-1)} \sum_{i, j=1, i \neq j}^{n}\left\langle R\left(Y_{i}, Y_{j}\right) Y_{i}, Y_{j}\right\rangle \\
& =c+\frac{1}{n(n-1)} \sum_{i, j=1}^{n}\left(\left\langle\alpha\left(Y_{i}, Y_{i}\right), \alpha\left(Y_{j}, Y_{j}\right)\right\rangle-\left|\alpha\left(Y_{i}, Y_{j}\right)\right|^{2}\right) .
\end{aligned}
$$

Observe que a curvatura seccional em relação a qualquer subespaço bi-dimensional de qualquer ponto em uma hipersuperfície regrada em $\mathbb{Q}^{n+1}(c)$ é sempre menor ou igual a $c$. De fato, seja $\sigma=\operatorname{span}\{X, Z\}$ um subespaço bi-dimensional de $T_{p} M^{n}$, tal que $X=\sum_{i=1}^{n} a_{i} Y_{i}$ e $Z=\sum_{i=1}^{n} b_{i} Y_{i}$ formam uma base ortonormal para $\sigma$, então

$$
\begin{aligned}
K(X, Z)= & c+\langle\alpha(X, X), \alpha(Z, Z)\rangle-|\alpha(X, Z)|^{2} \\
= & c-\sum_{j=1}^{n-1}\left(a_{j} b_{n}-a_{n} b_{j}\right)^{2}\left|\alpha\left(Y_{j}, Y_{n}\right)\right|^{2} \\
& +\sum_{j, i=1}^{n-1} j \neq i=1-a_{j} a_{i} b_{n}^{2}+a_{j} a_{n} b_{i} b_{n}+a_{i} a_{n} b_{j} b_{n}+ \\
& \left.-a_{n}^{2} b_{i} b_{j}\right)\left\langle\alpha\left(Y_{j}, Y_{n}\right), \alpha\left(Y_{i}, Y_{n}\right)\right\rangle \\
= & c-\left|\sum_{j=1}^{n-1}\left(a_{j} b_{n}-a_{n} b_{j}\right) \alpha\left(Y_{j}, Y_{n}\right)\right|^{2}
\end{aligned}
$$


Observação 5.5 Sejam $M^{n}, n \geq 3$, uma hipersuperfície conexa regrada em $\mathbb{Q}^{n+1}(c)$ e $p$ um ponto qualquer em $M^{n}$. A curvatura seccional da hipersuperfície em $p$ é diferente de $c$ se, e somente se, o índice de nulidade relativa em $p$ é igual a $n-2$.

Verifiquemos o fato anterior para o caso $c=1$, já que nos outros dois casos o raciocínio é análogo.

Tomemos novamente $\left\{Y_{1}, Y_{2}, \ldots . . Y_{n}\right\}$ uma base ortonormal para o espaço tangente a $M^{n}$ em $p$, tal que $Y_{j}, 1 \leq j \leq n-1$, são tangentes à folha que regra a hipersuperfície. Denotando por $A$ a matriz da segunda forma fundamental da hipersuperfície $M^{n}$, temos que

$$
A=\left(\begin{array}{ccccc}
\left\langle A Y_{1}, Y_{1}\right\rangle & \left\langle A Y_{1}, Y_{2}\right\rangle & \left\langle A Y_{1}, Y_{3}\right\rangle & \cdots & \left\langle A Y_{1}, Y_{n}\right\rangle \\
\left\langle A Y_{2}, Y_{1}\right\rangle & \left\langle A Y_{2}, Y_{2}\right\rangle & \left\langle A Y_{2}, Y_{3}\right\rangle & \cdots & \left\langle A Y_{2}, Y_{n}\right\rangle \\
\vdots & \vdots & \vdots & \vdots & \vdots \\
\left\langle A Y_{n}, Y_{1}\right\rangle & \left\langle A Y_{n}, Y_{2}\right\rangle & \left\langle A Y_{n}, Y_{3}\right\rangle & \cdots & \left\langle A Y_{n}, Y_{n}\right\rangle
\end{array}\right)
$$

ou equivalentemente, usando-se o fato que os $\alpha\left(Y_{i}, Y_{j}\right)=0,1 \leq i, j \leq n-1$

$$
A=\left(\begin{array}{ccccc}
0 & 0 & 0 & \cdots & \left\langle A Y_{1}, Y_{n}\right\rangle \\
0 & 0 & \cdots & \cdots & \left\langle A Y_{2}, Y_{n}\right\rangle \\
\vdots & & & & \\
\left\langle A Y_{n}, Y_{1}\right\rangle & \left\langle A Y_{n}, Y_{2}\right\rangle & \left\langle A Y_{n}, Y_{3}\right\rangle & \cdots & \left\langle A Y_{n}, Y_{n}\right\rangle
\end{array}\right)
$$

Logo o postoA é menor ou igual a 2 , com

$$
\text { posto } A=\left\{\begin{array}{l}
2 \text { se } \nu=n-2, \\
1 \text { se } \nu=n-1, \\
0 \text { se } \nu=n .
\end{array}\right.
$$

A igualdade anterior segue-se do fato que posto $A=n-\operatorname{dim}(\operatorname{Ker} A)$ e $\operatorname{dim}(\operatorname{Ker} A)$ $=\operatorname{dim}(\operatorname{Ker} \alpha)$.

Suponhamos então que $p$ seja um ponto em $M^{n}$ tal que a curvatura seccional da hipersuperfície em $p$ seja diferente de 1 , isto é, existe pelo menos um subespaço, $\sigma$, bi-dimensional do espaço tangente a $M^{n}$ em $p$ tal que a curvatura extrínseca da hipersuperfície calculada em $\sigma$ é diferente de zero, isto é, usando-se (5.6) e (5.7) temos que existe pelo menos um $Y_{j}$ tal que $\left|\alpha\left(Y_{j}, Y_{n}\right)\right|^{2} \neq 0$, ou equivalentemente, $\left\langle A Y_{j}, Y_{n}\right\rangle^{2} \neq 0$. Logo neste caso, temos que posto $A=2$, donde segue-se o desejado.

Reciprocamente, seja $M^{n}$ uma hipersuperfície regrada conexa com $\nu=n-2$ e tomemos $\left\{Z_{1}, Z_{2}, \ldots . Z_{n}\right\}$ uma base ortonormal para o espaço tangente a $M^{n}$ em $p$, tal que $Z_{j}, 1 \leq j \leq n-2$ geram a nulidade relativa de $M^{n}$ em $p$, isto é, 


$$
A=\left(\begin{array}{ccccc}
0 & 0 & \cdots & \ldots & 0 \\
\vdots & \vdots & \ldots & \ldots & 0 \\
0 & 0 & \cdots & \left\langle A Z_{n-1}, Z_{n-1}\right\rangle & \left\langle A Z_{n-1}, Z_{n}\right\rangle \\
0 & 0 & \cdots & \left\langle A Z_{n}, Z_{n-1}\right\rangle & \left\langle A Z_{n}, Z_{n}\right\rangle
\end{array}\right)
$$

Como o posto $A=2$, segue-se o desejado.

\subsection{Classificação das hipersuperfícies regradas e de Weingarten em $\mathbb{S}^{n+1}$}

Consideraremos, no que se segue, $M^{n} \subset \mathbb{S}^{n+1}$ uma hipersuperfície conexa regrada orientada com índice de nulidade relativa constante, $\nu=n-2$, ou seja, existe uma imersão isométrica $g: V^{2} \rightarrow \mathbb{S}^{n+1}$ bem definida e $M^{n}$ pode ser parametrizada com em (5.2). Tomemos ainda, $e_{1}, e_{2}, \ldots . . e_{n+1}, n+1$ campos ortonormais localmente definidos em $V^{2}$, tais que $e_{1}(y)$ e $e_{2}(y)$ são tangentes à imersão $g$ em $y$ e $e_{3}(y), e_{4}(y), \ldots e_{n+1}(y)$ geram o espaço normal à imersão $g$ em $\mathbb{S}^{n+1}$. A seguir demonstraremos alguns resultados que são fundamentais para o teorema principal desta seção.

Observamos, em primeiro lugar, que se a imersão $f: M^{n} \rightarrow \mathbb{S}^{n+1}$ define uma hipersuperfície regrada conexa e de Weingarten $\operatorname{com} \nu=n-2$ então mostra-se que o cone sobre $f$, isto é, a hipersuperfície $N^{n+1}$ definida através da imersão

$$
\begin{gathered}
F: \mathbb{R}_{+} \times M^{n} \longrightarrow \mathbb{R}^{n+2} \\
(t, p) \longrightarrow t f(p)
\end{gathered}
$$

é uma hipersuperfície regrada conexa $\operatorname{com} \nu=(n+1)-2$. Observamos ainda, que a aplicação de Gauss de $N^{n+1}$ em $\mathbb{S}^{n+1}$ coincide com a aplicação de Gauss de $M^{n} \mathrm{em}$ $\mathbb{S}^{n+1}$, pois em ambos os casos a aplicação de Gauss é paralela ao longo da nulidade relativa, o que significa que ela depende apenas do que não pertence à nulidade relativa, e estes subespaços coincidem em $f(p)$ e $F(t, p) \forall t \in \mathbb{R}_{+}$.

Pela observação anterior, usando-se o lema

Lema 5.6 Seja $M^{n} \subset \mathbb{R}^{n+1}$ uma hipersuperficie regrada com indice de nulidade relativa $\nu=n-2$. Então a imersão $g$ define uma superfície regrada em $\mathbb{S}^{n}$.

cuja demonstração encontra-se em [DT] e observando-se que a imersão g parametriza a aplicação de Gauss, fica demonstrada a seguinte proposição. 
Proposição 5.7 Seja $M^{n} \subset \mathbb{S}^{n+1}$ uma hipersuperfície regrada conexa orientada com $\nu=n-2$. Então a imersão $g$ é uma superfície regrada em $\mathbb{S}^{n+1}$.

Segue-se pela proposição anterior, que os $n+1$ campos ortonormais escolhidos, localmente definidos em $V^{2}$, podem ser tomados de forma que $e_{1}(y)$ seja tangente à folha da imersão $g$ passando por $y$, isto é, $\theta\left(e_{1}, e_{1}\right)=0$ onde $\theta$ é a segunda forma fundamental da imersão $g$. Com relação a esta base temos que

$$
B_{e_{j}}(y)=B_{j}=\left(\begin{array}{cc}
0 & \left\langle\theta\left(e_{1}, e_{2}\right), e_{j}\right\rangle \\
\left\langle\theta\left(e_{1}, e_{2}\right), e_{j}\right\rangle & \left\langle\theta\left(e_{2}, e_{2}\right), e_{j}\right\rangle
\end{array}\right)=\left(\begin{array}{cc}
0 & \beta_{j} \\
\beta_{j} & \lambda_{j}
\end{array}\right),
$$

$\operatorname{com} 3 \leq j \leq(n+1)$

Tomando-se $\left\{Y_{1}, Y_{2}, \ldots . Y_{n}\right\}$ uma base ortonormal para o espaço tangente a $M^{n}$ no ponto $(y, w)$, tal que $Y_{j}, 1 \leq j \leq n-2$ geram a nulidade relativa de $M^{n}$ em $(y, w)$, segue-se por (5.3) que a curvatura média, $H$, e a curvatura escalar, $S$, de $M^{n} \mathrm{em}$ $(y, w)$ são dadas por:

$$
\begin{gathered}
H(y, w)=\frac{1}{n} \operatorname{tr} A=\frac{1}{n} \operatorname{tr} B_{w}^{-1}(y)=\frac{1}{n} \frac{\operatorname{tr} B_{w}(y)}{\operatorname{det} B_{w}(y)} . \\
S(y, w)=\frac{1}{n(n-1)} \sum_{i, j=1, i \neq j}^{n}\left\langle R\left(Y_{i}, Y_{j}\right) Y_{i}, Y_{j}\right\rangle \\
=1+\frac{1}{n(n-1)} \sum_{i, j=1}^{n}\left(\left\langle\alpha\left(Y_{i}, Y_{i}\right), \alpha\left(Y_{j}, Y_{j}\right)\right\rangle-\left|\alpha\left(Y_{i}, Y_{j}\right)\right|^{2}\right) \\
=1+\frac{1}{n(n-1)} \sum_{i, j=n-1}^{n}\left(\left\langle\alpha\left(Y_{i}, Y_{i}\right), \alpha\left(Y_{j}, Y_{j}\right)\right\rangle-\left|\alpha\left(Y_{i}, Y_{j}\right)\right|^{2}\right) \\
=1+\frac{2}{n(n-1)}\left(\operatorname{det} B_{w}^{-1}(y)\right) \\
=1+\frac{2}{n(n-1)}\left(\frac{1}{\operatorname{det} B_{w}(y)}\right) .
\end{gathered}
$$

Observação 5.8 Sejam $M^{n} \subset \mathbb{S}^{n+1}$ uma hipersuperfície regrada e de Weingarten com índice de nulidade relativa constante $\nu=n-2$ e $(y, w) \sim\left(y_{1}, y_{2}, l_{3}, l_{4}, \ldots . l_{n+1}\right)$ uma parametrização local de $M^{n}$ onde $y_{1}$ e $y_{2}$ são as coordenadas de $y$ e $w=\sum_{j=3}^{n+1} l_{j} e_{j}$ é um campo normal unitário à imersão $g$. Note que $l_{j}=l_{j}(y, w)$ e $\sum_{j=3}^{n+1} l_{j}^{2}=1$, e $e_{1}, e_{2}, \ldots e_{n+1}$, são $n+1$ campos tomados como anteriormente. Usando-se a notação dada em (5.9), mostra-se que

$$
d\left(\sum_{j=3}^{n+1} l_{j} \lambda_{j}\right) \wedge d\left(\sum_{j=3}^{n+1} l_{j} \beta_{j}\right)=0 .
$$


De fato, segue-se por (1.14) que se $M^{n}$ é de Weingarten então $d H \wedge d S=0$. Utilizando-se (5.10) e (5.11) e escrevendo $B_{w}(y)=B$, temos

$$
\begin{aligned}
d H \wedge d S=0 & \Leftrightarrow\left(\frac{d(\operatorname{tr} B) \operatorname{det} B-d(\operatorname{det} B) \operatorname{tr} B}{n(\operatorname{det} B)^{2}}\right) \wedge\left(\frac{-2 d(\operatorname{det} B)}{(n(n-1))(\operatorname{det} B)^{2}}\right)=0 \\
& \Leftrightarrow d(\operatorname{tr} B) \operatorname{det} B \wedge(-2) d(\operatorname{det} B)=0 \\
& \Leftrightarrow d(\operatorname{tr} B) \wedge d(\operatorname{det} B)=0 .
\end{aligned}
$$

Agora ainda por (5.9) temos que

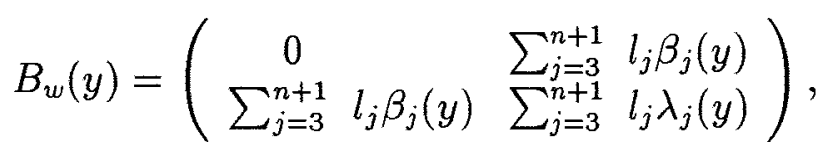

donde segue-se o desejado.

Lema 5.9 Seja $M^{n} \subset \mathbb{S}^{n+1}$ uma hipersuperficie regrada e de Weingarten com $\nu=$ $n-2$. Então a dimensão do primeiro espaço normal da imersão g é constante igual $a 1$.

\section{Demonstração:}

Como $M^{n}$ é uma hipersuperfície regrada segue-se, pela Proposição (5.7), que $g$ define uma superfície regrada em $\mathbb{S}^{n+1}$.

Consideremos, como antes, $e_{1}, e_{2}, e_{3}, \ldots \ldots e_{n+1}, n+1$ campos ortonormais tais que $e_{1}(y), e_{2}(y)$ geram o espaço tangente à imersão $g$ em $y \operatorname{com} \theta\left(e_{1}, e_{1}\right)=0 \mathrm{e}$ $e_{3}(y), \ldots \ldots e_{n+1}(y)$ geram o espaço normal a imersão $g$ em $\mathbb{S}^{n+1}$. Denotando por $N_{1}^{g}(y)$ o primeiro espaço normal da imersão $g$ em $y$, temos que $N_{1}^{g}(y)=\operatorname{span}\left\{\theta\left(e_{1}, e_{2}\right), \theta\left(e_{2}, e_{2}\right)\right\}$, isto é, $\operatorname{dim} N_{1}^{g} \leq 2$.

Suponhamos que $\operatorname{dim} N_{1}^{g}(y)=2$, portanto em uma vizinhança de $y$ vale que $\operatorname{dim} N_{1}^{g}=2$. Suponhamos ainda que nesta vizinhança $e_{3}, e_{4}$ geram $N_{1}^{g} \operatorname{com} e_{4}$ ortogonal a $\theta\left(e_{1}, e_{2}\right)$. Temos então, neste caso, que a segunda forma fundamental da imersão $g$ é dada por

$$
B_{3}=\left(\begin{array}{cc}
0 & \beta_{3} \\
\beta_{3} & \lambda_{3}
\end{array}\right), \quad B_{4}=\left(\begin{array}{cc}
0 & 0 \\
0 & \lambda_{4}
\end{array}\right),
$$

$\mathrm{e}$

$$
B_{j}=0, \quad 5 \leq j \leq n+1 .
$$

Como, por hipótese, $M^{n}$ é de Weingarten, segue-se por (5.12) que

$$
d\left(l_{3} \lambda_{3}+l_{4} \lambda_{4}\right) \wedge d\left(l_{3} \beta_{3}\right)=0
$$


ou seja,

$$
\left(\lambda_{3} d l_{3}+l_{3} d \lambda_{3}+\lambda_{4} d l_{4}+l_{4} d \lambda_{4}\right) \wedge\left(\beta_{3} d l_{3}+l_{3} d \beta_{3}\right)=0 .
$$

Observando-se que $\beta_{j^{\prime} s}$ e $\lambda_{j^{\prime} s}$ são funções apenas da variável $y$ segue-se, aplicando a última igualdade ao par $\left(\frac{\partial}{\partial l_{4}}, \frac{\partial}{\partial l_{3}}\right)$, que $\lambda_{4} \beta_{3}=0$. Se $\beta_{3}=0$, então $\theta\left(e_{1}, e_{2}\right)=0$, o que implica que $\operatorname{dim} N_{1}^{g}<2$; se $\lambda_{4}=0$, então $B_{4}=0$ o que implica novamente que $\operatorname{dim} N_{1}^{g}<2$. Logo $\operatorname{dim} N_{1}^{g} \leq 1$.

Suponhamos agora que $\operatorname{dim} N_{1}^{g}(y)=0$, isto é, $g$ determina um imersão totalmente geodésica em $y$. Neste caso, temos que

$$
B_{j}=0, \quad 3 \leq j \leq n+1,
$$

donde segue-se que $B_{v}$ é singular na direção de qualquer $v=\sum_{j=3}^{n+1} l_{j} e_{j}$ e portanto pelo Corolário (5.3) $\psi$ não determina uma imersão em $y$, o que gera uma contradição.

Logo, $\operatorname{dim} N_{1}^{g}=1$ em todo ponto.

Proposição 5.10 Seja $M^{n} \subset \mathbb{S}^{n+1}$ uma hipersuperfície conexa regrada de Weingarten com $\nu=n-2$. Então existe uma subvariedade totalmente geodésica $\mathbb{S}^{3} \subset \mathbb{S}^{n+1}$, tal que $g\left(V^{2}\right) \subset \mathbb{S}^{3}$ é uma superfície regrada e de Weingarten não desenvolvível e satisfaz $4 H^{2}+a^{2}(K-1)=0$, onde a é uma constante real, e $K$ e $H$ são as curvaturas Gaussiana e média da superfície, respectivamente.

\section{Demonstração:}

Por hipótese e usando a Proposição (5.7) e o Lema (5.9), segue-se que $g$ determina uma superfície regrada em $\mathbb{S}^{n+1}$ tal que $\operatorname{dim} N_{1}^{g}=1$ em todo ponto. Tomando-se como antes, $e_{1}, \ldots . e_{n+1}$ uma base ortonormal ao longo de $V^{2}$, suponhamos que $e_{3}$ gera $N_{1}^{g}$, ou seja

$$
B_{3}=\left(\begin{array}{cc}
0 & \beta \\
\beta & \lambda
\end{array}\right), \quad B_{j}=0,4 \leq j \leq n+1
$$

Queremos mostrar que $N_{1}^{g}$ é paralelo, pois neste caso teremos pelo Corolário (1.24) que a imersão $g$ reduz codimensão como desejado. Para isso basta mostrar que $\left(N_{1}^{g}\right)^{\perp}$ é paralelo. De fato, se $\left(N_{1}^{g}\right)^{\perp}$ é paralelo então denotando $\eta=\sum_{k=4}^{n+1} t_{k} e_{k}$ segue-se que

$$
\begin{aligned}
\left\langle\eta, e_{3}\right\rangle=0 & \Rightarrow\left\langle\eta, \nabla_{e_{j}}^{\perp} e_{3}\right\rangle=0, \quad j=1,2 \\
& \Rightarrow \nabla_{e_{j}}^{\perp} e_{3} \in N_{1}^{g} \\
& \Rightarrow N_{1}^{g} \text { é paralelo, }
\end{aligned}
$$


onde $\nabla$ é a conexão de Levi-Civita em $M^{n}$. Mostremos então que $\left(N_{1}^{g}\right)^{\perp}$ é paralelo.

Como estamos trabalhando em um espaço de curvatura constante, temos por (1.1) que a equação de Codazzi se reduz a

$$
\left(\nabla_{e_{1}} B\right)\left(e_{2}, \eta\right)=\left(\nabla_{e_{2}} B\right)\left(e_{1}, \eta\right)
$$

onde

$$
\left(\nabla_{e_{i}} B\right)\left(e_{j}, \eta\right)=\nabla_{e_{i}} B_{\eta} e_{j}-B_{\eta} \nabla_{e_{i}} e_{j}-B_{\nabla_{e_{i}}^{\frac{1}{\eta}}} e_{j}
$$

Em nosso caso, segue-se por (5.13) que

$$
B_{\nabla_{e_{1}} \eta} e_{2}=B_{\nabla_{e_{2}} \eta} e_{1}
$$

ou equivalentemente,

$$
\left\langle B_{\nabla_{e_{1}} \eta} e_{2}, e_{1}\right\rangle e_{1}+\left\langle B_{\nabla \frac{1}{e_{1}} \eta} e_{2}, e_{2}\right\rangle e_{2}-\left\langle B_{\nabla \frac{1}{e_{2}} \eta} e_{1}, e_{2}\right\rangle e_{2}=0
$$

isto é,

$$
\left\{\begin{array}{l}
\left\langle B_{\nabla_{e_{1}} \eta} e_{2}, e_{1}\right\rangle=0, \\
\left.B_{\nabla_{e_{1}} \frac{1}{1} \eta} e_{2}, e_{2}\right\rangle-\left\langle B_{\nabla_{e_{2}} \eta} e_{1}, e_{2}\right\rangle=0 .
\end{array}\right.
$$

Observando-se que para $i, j, k=1,2$

$$
\begin{aligned}
\left\langle B_{\nabla_{e_{j}}, \eta} e_{i}, e_{k}\right\rangle= & \left\langle\theta\left(e_{i}, e_{k}\right), \nabla_{e_{j}}^{\perp} \eta\right\rangle \\
& =\left\langle\theta\left(e_{i}, e_{k}\right), \sum_{l=3}^{n}\left\langle\nabla_{e_{j}}^{\perp} \eta, e_{l}\right\rangle e_{l}\right\rangle \\
& =\left\langle\theta\left(e_{i}, e_{k}\right), e_{3}\right\rangle\left\langle\nabla_{e_{j}}^{\perp} \eta, e_{3}\right\rangle
\end{aligned}
$$

temos que o sistema anterior é equivalente a

$$
\left\{\begin{array}{l}
\beta\left\langle\nabla_{e_{1}}^{\perp} \eta, e_{3}\right\rangle=0, \\
\lambda\left\langle\nabla_{e_{1}}^{\perp} \eta, e_{3}\right\rangle-\beta\left\langle\nabla_{e_{2}}^{\perp} \eta, e_{3}\right\rangle=0,
\end{array}\right.
$$

donde segue-se que

$$
\left\langle\nabla_{e_{1}}^{\perp} \eta, e_{3}\right\rangle=\left\langle\nabla_{e_{2}}^{\perp} \eta, e_{3}\right\rangle=0
$$

pois caso contrário, $B_{v}$ seria singular na direção de qualquer $v$, o que geraria uma contradição pelo Corolário (5.3). Logo $\left(N_{1}^{g}\right)^{\perp}$ é paralelo, e portanto pelo Corolário (1.24) existe uma subvariedade totalmente geodésica $\mathbb{S}^{3} \subset \mathbb{S}^{n+1}$, tal que $g\left(V^{2}\right) \subset \mathbb{S}^{3}$.

Como $M^{n}$ é de Weingarten, segue-se por (5.12) e (5.13) que

$$
d(l \lambda) \wedge d(l \beta)=0
$$


Aplicando-se este produto exterior ao par $\left(e_{j}, \frac{\partial}{\partial l}\right), j=1,2$, temos que

$$
l\left(\beta d \lambda\left(e_{j}\right)-\lambda d \beta\left(e_{j}\right)\right)=0 \forall l \in \mathbb{R} .
$$

Portanto

$$
\beta d \lambda-\lambda d \beta=0
$$

donde segue-se que existe uma constante real, $a$, tal que

$$
\lambda=a \beta .
$$

Temos por (5.13) que as curvaturas Gaussiana e média da imersão $g$ verificam, respectivamente,

$$
K=1-\beta^{2}, \quad H=\frac{1}{2} \lambda
$$

donde segue-se por (5.14) que a imersão $g$ satisfaz a relação

$$
4 H^{2}+a^{2}(K-1)=0 .
$$

A próxima proposição pode ser encontrada em [DT].

Proposição 5.11 Seja $g: V^{2} \longrightarrow \mathbb{S}^{3}$ uma superfície regrada conexa não desenvolvivel em $\mathbb{S}^{3}$ tal que $4 H^{2}+a^{2}(K-1)=0$. Então $H=0$ ou $H=\frac{a}{2} \neq 0$ e $K=0$. No segundo caso, temos que a superfície está contida no produto de dois círculos.

\section{Demonstração:}

Se a constante $a$ for nula, então $H=0$. Caso contrário, mostraremos que $H=\frac{a}{2}$ e $K=0$.

Como $g\left(V^{2}\right) \subset \mathbb{S}^{3}$ é uma superfície regrada, podemos parametrizá-la localmente por

$$
Y(s, t)=\beta_{0}(s) \cos t+\beta_{1}(s) \sin t
$$

onde $\beta_{0}$ e $\beta_{1}$ são vetores em $\mathbb{R}^{4}$ tais que

$$
\left|\beta_{0}\right|=\left|\beta_{1}\right|=1, \quad\left\langle\beta_{0}, \beta_{1}\right\rangle=0, \quad\left\langle\dot{\beta}_{0}, \dot{\beta}_{1}\right\rangle=0
$$

Além disso, podemos tomar $s$ o parâmetro comprimento de arco de $\beta_{1}$, isto é , $\left|\dot{\beta}_{1}\right|=1$. Observe que esta parametrização não coincide com a parametrização usada por nós nos capítulos anteriores. 
Tomemos a base ortonormal $\left\{Y_{t}, \frac{\tilde{Y}_{s}}{\left|\tilde{Y}_{s}\right|}, Y_{t} \wedge \frac{\tilde{Y}_{s}}{\left|\tilde{Y}_{s}\right|} \wedge Y, Y\right\}$ de $\mathbb{R}^{4}$, onde $\tilde{Y}_{s}=Y_{s}$ $\left\langle Y_{t}, Y_{s}\right\rangle Y_{t}$. Usando-se cálculos análogos aos feitos na seção (2.2) e denotando

$$
\left\{\begin{array}{l}
A(s)=\left|\dot{\beta}_{0}\right|^{2}-\left\langle\dot{\beta}_{0}, \beta_{1}\right\rangle^{2} \\
B(s)=1-\left\langle\dot{\beta}_{0}, \beta_{1}\right\rangle^{2} \\
P(s, t)=A(s) \cos ^{2} t+B(s) \sin ^{2} t \\
Q(s, t)=\left\langle\dot{\beta}_{1}, \dot{\beta}_{0} \wedge \beta_{1} \wedge \beta_{0}\right\rangle \\
h(s, t)=F(s) \cos ^{2} t+D(s) \sin ^{2} t+(L(s)+G(s)) \cos t \sin t
\end{array}\right.
$$

onde $F(s), D(s), L(s)$ e $G(s)$ são dados em (2.10), mostramos que as curvaturas Gaussiana e média da superfície são dadas por

$$
K=1-\frac{Q^{2}}{P^{2}}, \quad H=\frac{h+2 Q\left\langle\dot{\beta}_{0}, \beta_{1}\right\rangle}{2 P^{\frac{3}{2}}} .
$$

Observa-se que $Q^{2}=A B$. De fato,

$$
\begin{aligned}
Q^{2} & =\left(\operatorname{det}\left[\begin{array}{c}
\dot{\beta}_{1} \\
\dot{\beta}_{0} \\
\beta_{1} \\
\beta_{0}
\end{array}\right]\right)= \\
& =\operatorname{det}\left(\left[\begin{array}{cccc}
2 & 0 & 0 & \left\langle\dot{\beta}_{1}, \beta_{0}\right\rangle \\
0 & \left|\dot{\beta}_{0}\right|^{2} & \left\langle\dot{\beta}_{0}, \beta_{1}\right\rangle & 0 \\
0 & \left\langle\dot{\beta}_{0}, \beta_{1}\right\rangle & 1 & 0 \\
\left\langle\dot{\beta}_{1}, \beta_{0}\right\rangle & 0 & 0 & 1
\end{array}\right]\right) \\
& =\left(\left|\dot{\beta}_{0}\right|^{2}-\left\langle\dot{\beta}_{0}, \beta_{1}\right\rangle^{2}\right)\left(1-\left\langle\dot{\beta}_{0}, \beta_{1}\right\rangle^{2}\right) \\
& =A B
\end{aligned}
$$

Segue-se então que na vizinhança de um ponto onde $K \neq 1, A(s) \neq 0$ e $B(s) \neq 0$ (observe que tal vizinhança existe, pois g é uma superfície regrada não desenvolvível).

Por hipótese, temos que a superfície satisfaz a relação $4 H^{2}+a^{2}(K-1)=0$, ou seja,

$$
4\left(\frac{h+2 Q\left\langle\dot{\beta}_{0}, \beta_{1}\right\rangle}{2 P^{\frac{3}{2}}}\right)^{2}=\frac{a^{2} Q^{2}}{P^{2}}, \quad \forall s, t
$$


donde segue-se, sem perda de generalidade, que

$$
h+2 Q\left\langle\dot{\beta}_{0}, \beta_{1}\right\rangle-a Q P^{\frac{1}{2}}=0, \quad \forall s, t .
$$

Derivando-se a igualdade (5.17) em $t$, segue-se que

$$
\frac{\partial h}{\partial t}-a Q \frac{1}{2 P^{\frac{1}{2}}} \frac{\partial P}{\partial t}=0
$$

onde

$$
\frac{\partial h}{\partial t}=\sin 2 t(D-F)+\cos 2 t(L+G)
$$

$\mathrm{e}$

$$
\frac{\partial P}{\partial t}=\sin 2 t(B-A)
$$

Como as funções $\sin 2 t$ e $\cos 2 t$ são linearmente independentes como funções de $t$, segue-se por (5.18) que $L+G=0$, ou seja, a equação (5.18) se reduz a

$$
\sin 2 t(D-F)-a Q \frac{1}{2 P^{\frac{1}{2}}} \frac{\partial P}{\partial t}=0
$$

Suponhamos que na vizinhança, $V$, de um ponto onde $K \neq 1$, existe um ponto tal que $\frac{\partial P}{\partial t} \neq 0$. Então em uma vizinhança, $U \subset V$, deste ponto tal que $\frac{\partial P}{\partial t} \neq 0 \mathrm{em}$ $U$, vale que

$$
\frac{1}{B-A}(D-F)-a Q \frac{1}{2 P^{\frac{1}{2}}}=0
$$

ou equivalentemente,

$$
\frac{1}{P^{\frac{1}{2}}}=\frac{2}{a Q(B-A)}(D-F) \text {. }
$$

$O$ segundo termo da igualdade anterior é função apenas do parâmetro $s$. Derivandose esta igualdade em $t$ na vizinhança $U$, segue-se que $\frac{\partial P}{\partial t}=0$ em $U$, contradição. Logo

$$
\frac{\partial P}{\partial t}=0, \quad \forall s, t \text { em } V \Rightarrow A(s)=B(s), \quad \text { em } V .
$$

Portanto por (5.15) e (5.16) temos que $K=0$ em $V$, donde segue-se que $H=\frac{a}{2}$ em $V$. Seja $W=\left\{p \in g\left(V^{2}\right): K(p) \neq 1\right\}$. Pelo o que foi feito anteriormente, temos que $H=\frac{a}{2}$ em $W$, mostremos que $W=g\left(V^{2}\right)$. Suponhamos que $W$ é conexo e $W \varsubsetneqq g\left(V^{2}\right)$, então existe um ponto $q \in \partial W$ tal que $K(q)=1$, o que é um absurdo pois neste caso existiria um seqüencia constante não nula tendendo para zero, observe que 
$H(q)=0$. Se $W$ não for conexo, chegamos a mesma contradição em cada componente conexa de $W$. Logo $H=\frac{a}{2}$ em toda a superfície, como queríamos demonstrar.

Para a segunda parte da proposição ver [S].

Neste ponto convém observar que decorre das proposições (5.10) e (5.11) o seguinte: Se $M^{n} \subset \mathbb{S}^{n+1}$ é uma hipersuperfície regrada conexa e de Weingarten com $\nu=n-2$, então existe uma subvariedade totalmente geodésica $\mathbb{S}^{3} \subset \mathbb{S}^{n+1}$ tal que $g\left(V^{2}\right) \subset \mathbb{S}^{3}$ é uma superfície regrada mínima ou está contida no produto de dois círculos.

Vamos fornecer agora, em cada caso, parametrizações explícitas para $g\left(V^{2}\right)$ e $M^{n}$, que serão utilizadas no teorema principal.

- Primeiro caso:

Suponha que $g\left(V^{2}\right)$ é uma superfície regrada mínima em $\mathbb{S}^{3}$. Então segue-se por [L] que $g\left(V^{2}\right)$ pode ser localmente parametrizada por

$$
g\left(t_{1}, t_{2}\right)=\beta_{1}\left(t_{2}\right) \cos t_{1}+\beta_{2}\left(t_{2}\right) \sin t_{1}
$$

onde, sem perda de generalidade, podemos tomar

$$
\beta_{1}\left(t_{2}\right)=\left(\cos a t_{2}, \sin a t_{2}, 0 \ldots 0\right)
$$

$\mathrm{e}$

$$
\beta_{2}\left(t_{2}\right)=\left(0,0, \cos t_{2}, \sin t_{2}, 0 \ldots 0\right),
$$

com $a$ uma constante real positiva.

Consideremos $\left\{e_{1}, e_{2}, e_{3}, \ldots . . e_{n+1}\right\}$ uma base ortonormal ao longo de $g\left(V^{2}\right)$ onde $e_{1}, e_{2}$ são tangentes à superfície, $e_{3}$ é normal à superfície e tangente a $\mathbb{S}^{3}$ e $e_{j}, 4 \leq$ $j \leq n+1$, são normais à superfície mas tangentes a $\mathbb{S}^{n+1}$. Explicitamente, tomemos:

$$
\begin{aligned}
& e_{1}=\frac{\partial g}{\partial t_{1}}=-\beta_{1}\left(t_{2}\right) \sin t_{1}+\beta_{2}\left(t_{2}\right) \cos t_{1}, \\
& e_{2}=\frac{1}{\sqrt{E}} \frac{\partial g}{\partial t_{2}}=\frac{1}{\sqrt{E}}\left(\dot{\beta}_{1}\left(t_{2}\right) \cos t_{1}+\dot{\beta}_{2}\left(t_{2}\right) \sin t_{1}\right), \\
& e_{3}=\frac{1}{\sqrt{E}}\left(-\frac{\dot{\beta}_{1}\left(t_{2}\right)}{a} \sin t_{1}+a \dot{\beta}_{2}\left(t_{2}\right) \cos t_{1}\right), \\
& e_{4}=(0,0,0,0,1,0 \ldots 0), \\
& e_{5}=(0,0,0,0,0,1,0 \ldots 0) \\
& e_{n+1}=(0 \ldots .0,1)
\end{aligned}
$$

onde

$$
\begin{aligned}
E & =a^{2} \cos ^{2} t_{1}+\sin ^{2} t_{1} \\
\dot{\beta}_{1}\left(t_{2}\right) & =a\left(-\sin a t_{2}, \cos a t_{2}, 0 \ldots 0\right) \\
\dot{\beta}_{2}\left(t_{2}\right) & =\left(0,0,-\sin t_{2}, \cos t_{2}, 0 \ldots 0\right)
\end{aligned}
$$


Denotando por $\bar{\nabla}$ e $\nabla$ as conexões de Levi-Civita de $\mathbb{S}^{n+1}$ e $\mathbb{R}^{n+2}$, respectivamente, determinemos a matriz da segunda forma fundamental da imersão $g$ com relação a base dada. Temos

$$
\begin{gathered}
\theta\left(e_{1}, e_{1}\right)=\left(\bar{\nabla}_{e_{1}} e_{1}\right)^{\perp}=\nabla_{e_{1}} e_{1}-\left\langle\nabla_{e_{1}} e_{1}, e_{1}\right\rangle e_{1}+ \\
-\left\langle\nabla_{e_{1}} e_{1}, e_{2}\right\rangle e_{2}-\left\langle\nabla_{e_{1}} e_{1}, g\right\rangle g .
\end{gathered}
$$

Como $\nabla_{e_{1}} e_{1}=\frac{\partial^{2} g}{\partial t_{1}^{2}}$, então

$$
\theta\left(e_{1}, e_{1}\right)=\overrightarrow{0}
$$

onde $\overrightarrow{0}$ é o vetor nulo em $\mathbb{R}^{n+2}$. Temos também que

$$
\theta\left(e_{2}, e_{2}\right)=\nabla_{e_{2}} e_{2}-\left\langle\nabla_{e_{2}} e_{2}, e_{1}\right\rangle e_{1}-\left\langle\nabla_{e_{2}} e_{2}, e_{2}\right\rangle e_{2}-\left\langle\nabla_{e_{2}} e_{2}, g\right\rangle g
$$

e como $\nabla_{e_{2}} e_{2}=\frac{1}{\sqrt{E}}\left(\frac{1}{\sqrt{E}} \frac{\partial^{2} g}{\partial t_{2}^{2}}+\frac{\partial}{\partial t_{2}}\left[\frac{1}{\sqrt{E}}\right] \frac{\partial g}{\partial t_{2}}\right)$ segue-se que,

$$
\begin{aligned}
\theta\left(e_{2}, e_{2}\right) & =\frac{1}{E}\left(\frac{\partial^{2} g}{\partial t_{2}^{2}}-\left\langle\frac{\partial^{2} g}{\partial t_{2}^{2}}, e_{1}\right\rangle e_{1}-\left\langle\frac{\partial^{2} g}{\partial t_{2}^{2}}, e_{2}\right\rangle e_{2}-\left\langle\frac{\partial^{2} g}{\partial t_{2}^{2}}, g\right\rangle g\right) \\
& =\frac{1}{E}\left(\frac{\partial^{2} g}{\partial t_{2}^{2}}-\left\langle\frac{\partial^{2} g}{\partial t_{2}^{2}}, e_{1}\right\rangle e_{1}-\left\langle\frac{\partial^{2} g}{\partial t_{2}^{2}}, g\right\rangle g\right) \\
& =\overrightarrow{0} .
\end{aligned}
$$

Analogamente temos ainda que

$$
\begin{aligned}
\theta\left(e_{1}, e_{2}\right)= & \frac{1}{\sqrt{E}}\left(\frac{\partial^{2} g}{\partial t_{1} \partial t_{2}}-\left\langle\frac{\partial^{2} g}{\partial t_{1} \partial t_{2}}, e_{1}\right\rangle e_{1}-\left\langle\frac{\partial^{2} g}{\partial t_{1} \partial t_{2}}, e_{2}\right\rangle e_{2}-\left\langle\frac{\partial^{2} g}{\partial t_{1} \partial t_{2}}, g\right\rangle g\right) \\
= & \frac{1}{\sqrt{E}}\left(\frac{\partial^{2} g}{\partial t_{1} \partial t_{2}}-\left\langle\frac{\partial^{2} g}{\partial t_{1} \partial t_{2}}, e_{2}\right\rangle e_{2}\right) \\
= & \frac{1}{\sqrt{E}}\left(-\dot{\beta}_{1}\left(t_{2}\right) \sin t_{1}+\dot{\beta}_{2}\left(t_{2}\right) \cos t_{1}+\right. \\
& \left.-\frac{1}{E}\left(\left(\dot{\beta}_{1}\left(t_{2}\right) \cos t_{1}+\dot{\beta}_{2}\left(t_{2}\right) \sin t_{1}\right) \cos t_{1} \sin t_{1}\left(1-a^{2}\right)\right)\right)
\end{aligned}
$$

donde segue-se que

$$
B_{3}=\left(\begin{array}{cc}
0 & \frac{a}{E} \\
\frac{a}{E} & 0
\end{array}\right), \quad B_{j}=0,4 \leq j \leq n+1 .
$$

Pelo Corolário (5.3) temos que $M^{n}$ pode ser parametrizada por $\psi(y, w)=w$ onde $y \in g\left(V^{2}\right)$ e $w \in \Lambda^{1}$, melhor dizendo, podemos parametrizar $M^{n}$ por

$$
X\left(t_{1}, t_{2}, s_{1}, \ldots s_{n-2}\right)=\sum_{j=3}^{n+1} \varphi_{j}\left(s_{1}, \ldots . s_{n-2}\right) e_{j}\left(t_{1}, t_{2}\right),
$$


onde $w=\sum_{j=3}^{n+1} \varphi_{j} e_{j}$ e $\sum_{j=3}^{n+1} \varphi_{j}^{2}=1$. Observe que $X$ define um fibrado normal de $n-2$ esferas unitárias sobre $g\left(V^{2}\right)$.

Segue-se ainda, pelo Corolário (5.3), que a matriz da segunda forma fundamental da imersão $X$ no ponto $(y, w)$ restrito ao complemento ortogonal da nulidade relativa é dada por $B_{w}^{-1}(y)$, isto é,

$$
A_{g}=\left(\begin{array}{ll}
0 & \frac{a \varphi_{3}}{E} \\
\frac{a \varphi_{3}}{E} & 0
\end{array}\right)^{-1} .
$$

Temos então por (5.10) e (5.11) que as curvaturas média e escalar da hipersuperfície são dadas, respectivamente, por

$$
H=0, \quad S=1+\frac{-2}{n(n-1)} \frac{E^{2}}{a^{2} \varphi_{3}^{2}}
$$

isto é, $M^{n}$ é uma hipersuperfície regrada mínima em $\mathbb{S}^{n+1}$.

- Segundo caso:

Suponha agora que $g\left(V^{2}\right)$ é uma superfície regrada contida no produto de dois círculos em $\mathbb{S}^{3}$. Sem perda de generalidade, $g\left(V^{2}\right)$ pode ser localmente parametrizada por

$$
\begin{aligned}
g\left(t_{1}, t_{2}\right)= & r_{1}\left(\sin \frac{t_{1}}{r_{1}}, \cos \frac{t_{1}}{r_{1}}, 0, \ldots 0\right)+ \\
& +r_{2}\left(0,0, \sin \frac{t_{2}}{r_{2}}, \cos \frac{t_{2}}{r_{2}}, 0, \ldots .0\right),
\end{aligned}
$$

com $r_{1}^{2}+r_{2}^{2}=1, r_{1} \neq 0$ e $r_{2} \neq 0$.

Consideremos, analogamente ao caso anterior, $\left\{e_{1}, \ldots . e_{n+1}\right\}$ uma base ortonormal ao longo de $g\left(V^{2}\right)$ onde $e_{1}, e_{2}$ são tangentes à superfície, $e_{3}$ é normal à superfície e tangente a $\mathbb{S}^{3}$ e $e_{j}, 4 \leq j \leq n+1$, são normais à superfície mas tangentes a $\mathbb{S}^{n+1}$. Explicitamente tomemos:

$$
\begin{aligned}
e_{1} & =r_{1} \frac{\partial g}{\partial t_{1}}-r_{2} \frac{\partial g}{\partial t_{2}} \\
& =\left(r_{1} \cos \frac{t_{1}}{r_{1}},-r_{1} \sin \frac{t_{1}}{r_{1}},-r_{2} \cos \frac{t_{2}}{r_{2}}, r_{2} \sin \frac{t_{2}}{r_{2}}, 0, \ldots .0\right), \\
e_{2} & =r_{2} \frac{\partial g}{\partial t_{1}}+r_{1} \frac{\partial g}{\partial t_{2}} \\
& =\left(r_{2} \cos \frac{t_{1}}{r_{1}},-r_{2} \sin \frac{t_{1}}{r_{1}}, r_{1} \cos \frac{t_{2}}{r_{2}},-r_{1} \sin \frac{t_{2}}{r_{2}}, 0, \ldots .0\right), \\
e_{3} & =\left(-r_{2} \sin \frac{t_{1}}{r_{1}},-r_{2} \cos \frac{t_{1}}{r_{1}}, r_{1} \sin \frac{t_{2}}{r_{2}}, r_{1} \cos \frac{t_{2}}{r_{2}}, 0, \ldots 0\right), \\
e_{4} & =(0,0,0,0,1,0, \ldots .0), \\
e_{5} & =(0,0,0,0,0,1,0, \ldots \ldots 0), \\
e_{n+1} & =(0, \ldots .0,1) .
\end{aligned}
$$


Observe que $e_{j} \in \mathbb{R}^{n+2}, 1 \leq j \leq n+1$. Determinemos a matriz da segunda forma fundamental da imersão $g$ com relação a esta base, onde $\bar{\nabla}$ e $\nabla$ denotam as conexões de Levi-Civita de $\mathbb{S}^{n+1}$ e $\mathbb{R}^{n+2}$, respectivamente. Temos

$$
\begin{gathered}
\theta\left(e_{1}, e_{1}\right)=\left(\bar{\nabla}_{e_{1}} e_{1}\right)^{\perp}=\nabla_{e_{1}} e_{1}-\left\langle\nabla_{e_{1}} e_{1}, e_{1}\right\rangle e_{1} \\
-\left\langle\nabla_{e_{1}} e_{1}, e_{2}\right\rangle e_{2}-\left\langle\nabla_{e_{1}} e_{1}, g\right\rangle g
\end{gathered}
$$

onde $\nabla_{e_{1}} e_{1}=\left(r_{1}^{2} \frac{\partial^{2} g}{\partial t_{1}^{2}}+r_{2}^{2} \frac{\partial^{2} g}{\partial t_{2}^{2}}\right)$, então

$$
\theta\left(e_{1}, e_{1}\right)=\overrightarrow{0}
$$

Temos ainda que $\nabla_{e_{2}} e_{2}=r_{2}^{2} \frac{\partial^{2} g}{\partial t_{1}^{2}}+r_{1}^{2} \frac{\partial^{2} g}{\partial t_{2}^{2}}$ e $\nabla_{e_{1}} e_{2}=r_{1} r_{2}\left(\frac{\partial^{2} g}{\partial t_{1}^{2}}-\frac{\partial^{2} g}{\partial t_{2}^{2}}\right)$, donde segue-se que

$$
\begin{aligned}
\theta\left(e_{2}, e_{2}\right)= & \frac{-r_{2}^{2}}{r_{1}}\left(\sin \frac{t_{1}}{r_{1}}, \cos \frac{t_{1}}{r_{1}}, 0, \ldots \ldots 0\right)+ \\
& -\frac{-r_{1}^{2}}{r_{2}}\left(0,0, \sin \frac{t_{2}}{r_{2}}, \cos \frac{t_{2}}{r_{2}}, 0, \ldots \ldots 0\right)+g
\end{aligned}
$$

$\mathrm{e}$

$$
\begin{aligned}
\theta\left(e_{1}, e_{2}\right)= & -r_{2}\left(\sin \frac{t_{1}}{r_{1}}, \cos \frac{t_{1}}{r_{1}}, 0 \ldots \ldots 0\right)+ \\
& +r_{1}\left(0,0, \sin \frac{t_{2}}{r_{2}}, \cos \frac{t_{2}}{r_{2}}, 0, \ldots \ldots 0\right) .
\end{aligned}
$$

Temos então que

$$
B_{3}=\left(\begin{array}{ll}
0 & 1 \\
1 & \frac{r_{2}^{2}-r_{1}^{2}}{r_{1} r_{2}}
\end{array}\right), \quad B_{j}=0,4 \leq j \leq n+1 .
$$

Seguindo novamente raciocínio análogo ao caso anterior, podemos parametrizar $M^{n}$ por

$$
X\left(t_{1}, t_{2}, s_{1}, \ldots . s_{n-2}\right)=\sum_{j=3}^{n+1} \varphi_{j}\left(s_{1}, \ldots . s_{n-2}\right) e_{j}\left(t_{1}, t_{2}\right),
$$

onde $w=\sum_{j=3}^{n+1} \varphi_{j} e_{j}$ e $\sum_{j=3}^{n+1} \varphi_{j}^{2}=1$. Observe, novamente, que $X$ define um fibrado normal de $n-2$ esferas sobre $g\left(V^{2}\right)$.

Segue-se ainda, que a segunda forma fundamental da imersão $X$ no ponto $(y, w)$ restrito ao complemento ortogonal da nulidade relativa é dada por

$$
A_{g}=\left(\begin{array}{ll}
0 & \varphi_{3} \\
\varphi_{3} & \frac{\left(r_{2}^{2}-r_{1}^{2}\right) \varphi_{3}}{r_{1} r_{2}}
\end{array}\right)^{-1}
$$

Temos então por (5.10) e (5.11) que as curvaturas média e escalar da hipersuperfície são dadas, respectivamente, por

$$
H=\frac{1}{n} \frac{\left(r_{1}^{2}-r_{2}^{2}\right)}{\varphi_{3} r_{1} r_{2}}, \quad S=1+\frac{-2}{n(n-1)} \frac{1}{\varphi_{3}^{2}},
$$


isto é, $M^{n}$ possui curvatura média e escalar constantes em relação a dois de seus parâmetros.

Observação 5.12 Os dois casos estudados anteriormente tratam de hipersuperfícies regradas e de Weingarten, ou seja, para cada caso existe uma aplicação não trivial, $F$, tal que $F(H, S)=0$. No primeiro caso a aplicação $F(p, q)=p$ satisfaz à condição dada. Já no segundo caso, cálculos simples nos levam à aplicação $F(p, q)=\frac{\left(n r_{1} r_{2}\right)^{2}}{\left(r_{1}^{2}-r_{2}^{2}\right)^{2}} p^{2}+\frac{n(n-1)}{2}(q-1)$.

Pelo segundo caso estudado, fica fácil darmos exemplos de hipersuperfícies regradas de Weingarten em $\mathbb{S}^{n+1}$ que não sejam exemplos triviais, melhor dizendo, exemplos que não possuem curvatura escalar ou média constante.

Exemplo 5.13 Mostremos que a hipersuperfície em $\mathbb{S}^{4}$ parametrizada por

$$
X\left(t_{1}, t_{2}, t_{3}\right)=e_{1}\left(t_{1}, t_{2}\right) \cos t_{3}+e_{2}\left(t_{1}, t_{2}\right) \sin t_{3}
$$

define uma hipersuperfície regrada de Weingarten, $M^{3} \subset \mathbb{S}^{4}$ com índice de nulidade relativa constante igual a 1 , onde

$$
\begin{gathered}
e_{1}=\left(-r_{2} \sin \frac{t_{1}}{r_{1}},-r_{2} \cos \frac{t_{1}}{r_{1}}, r_{1} \sin \frac{t_{2}}{r_{2}}, r_{1} \cos \frac{t_{2}}{r_{2}}, 0\right), \\
e_{2}=(0,0,0,0,1),
\end{gathered}
$$

$\operatorname{com} r_{1}^{2}+r_{2}^{2}=1, r_{1} \neq 0$ e $r_{2} \neq 0$.

Consideremos $\left\{f_{1}, f_{2}, f_{3}\right\}$ o referencial ortonormal tangente a $M^{3}$, dado por:

$$
\begin{gathered}
f_{1}=\frac{r_{1} X_{t_{1}}-r_{2} X_{t_{2}}}{\left|r_{1} X_{t_{1}}-r_{2} X_{t_{2}}\right|}=\left(-r_{2} \cos \frac{t_{1}}{r_{1}}, r_{2} \sin \frac{t_{1}}{r_{1}},-r_{1} \cos \frac{t_{2}}{r_{2}}, r_{1} \sin \frac{t_{2}}{r_{2}}, 0\right), \\
f_{2}=\frac{\frac{r_{1}^{2}}{r_{2}} X_{t_{1}}+\frac{r_{2}^{2}}{r_{1}} X_{t_{2}}}{\left|\frac{r_{1}^{2}}{r_{2}} X_{t_{1}}+\frac{r_{2}^{2}}{r_{1}} X_{t_{2}}\right|}=\left(-r_{1} \cos \frac{t_{1}}{r_{1}}, r_{1} \sin \frac{t_{1}}{r_{1}}, r_{2} \cos \frac{t_{2}}{r_{2}},-r_{2} \sin \frac{t_{2}}{r_{2}}, 0\right), \\
f_{3}=X_{t_{3}} .
\end{gathered}
$$

Calculemos a matriz da segunda forma fundamental, com relação a este referencial. A normal à hipersuperfície é dada por

$$
\begin{aligned}
\eta & =f_{1} \wedge f_{3} \wedge f_{2} \wedge X \\
& =\left(r_{1} \sin \frac{t_{1}}{r_{1}}, r_{1} \cos \frac{t_{1}}{r_{1}}, r_{2} \sin \frac{t_{2}}{r_{2}}, r_{2} \cos \frac{t_{2}}{r_{2}}, 0\right)
\end{aligned}
$$


Observe que se considerarmos a aplicação $g$, dada em (5.24), com imagem em $\mathbb{S}^{4}$ teremos como esperado que $\eta=g$. Denotando por $\bar{\nabla}$ a conexão de Levi-Civita de $\mathbb{S}^{4}$, temos que:

$$
\begin{aligned}
& \alpha\left(f_{1}, f_{1}\right)=\frac{1}{\left|r_{1} X_{t_{1}}-r_{2} X_{t_{2}}\right|^{2}}\left(r_{1}^{2} \bar{\nabla}_{X_{t_{1}}} X_{t_{1}}+r_{2}^{2} \bar{\nabla}_{X_{t_{2}}} X_{t_{2}}\right)^{\perp}= \\
& =\frac{1}{\cos ^{2} t_{3}}\left(r_{1}^{2} X_{t_{1} t_{1}}+r_{2}^{2} X_{t_{2} t_{2}}+\right. \\
& \left.-\left(\cos t_{3} \sin t_{3}\right) f_{3}+\left(\cos ^{2} t_{3}\right) X\right) \\
& =\overrightarrow{0} \text {, } \\
& \alpha\left(f_{2}, f_{2}\right)=\frac{1}{\left|\frac{r_{1}^{2}}{r_{2}} X_{t_{1}}+\frac{r_{2}^{2}}{r_{1}} X_{t_{2}}\right|^{2}}\left(\frac{r_{1}^{4}}{r_{2}^{2}} \bar{\nabla}_{X_{t_{1}}} X_{t_{1}}+\frac{r_{2}^{4}}{r_{1}^{2}} \bar{\nabla}_{X_{t_{2}}} X_{t_{2}}\right)^{\perp}= \\
& =\frac{1}{\cos ^{2} t_{3}}\left(\frac{r_{1}^{4}}{r_{2}^{2}} X_{t_{1} t_{1}}+\frac{r_{2}^{4}}{r_{1}^{2}} X_{t_{2} t_{2}}+\right. \\
& \left.-\left(\cos t_{3} \sin t_{3}\right) f_{3}+\left(\cos ^{2} t_{3}\right) X\right) \\
& =\frac{1}{\cos ^{2} t_{3}}\left(\frac{1}{r_{2}}\left(r_{1}^{2}-r_{2}^{2}\right) \cos t_{3} \sin \frac{t_{1}}{r_{1}}\right. \text {, } \\
& \frac{1}{r_{2}}\left(r_{1}^{2}-r_{2}^{2}\right) \cos t_{3} \cos \frac{t_{1}}{r_{1}} \text {, } \\
& \frac{1}{r_{1}}\left(r_{1}^{2}-r_{2}^{2}\right) \cos t_{3} \sin \frac{t_{2}}{r_{2}} \\
& \left.\frac{1}{r_{1}}\left(r_{1}^{2}-r_{2}^{2}\right) \cos t_{3} \cos \frac{t_{2}}{r_{2}}, 0\right) \\
& =\frac{1}{\cos t_{3}}\left(\frac{1}{r_{2}}\left(r_{1}^{2}-r_{2}^{2}\right) \sin \frac{t_{1}}{r_{1}}, \frac{1}{r_{2}}\left(r_{1}^{2}-r_{2}^{2}\right) \cos \frac{t_{1}}{r_{1}}\right. \text {, } \\
& \left.\frac{1}{r_{1}}\left(r_{1}^{2}-r_{2}^{2}\right) \sin \frac{t_{2}}{r_{2}}, \frac{1}{r_{1}}\left(r_{1}^{2}-r_{2}^{2}\right) \cos \frac{t_{2}}{r_{2}}, 0\right) \text {, } \\
& \alpha\left(f_{3}, f_{3}\right)=\left(\bar{\nabla}_{X_{t_{3}}} X_{t_{3}}\right)^{\perp} \\
& =X_{t_{3} t_{3}}-\sum_{j=1}^{j=3}\left\langle X_{t_{3} t_{3}}, f_{j}\right\rangle f_{j}-\left\langle X_{t_{3} t_{3}}, X\right\rangle X \\
& =X_{t_{3} t_{3}}+X \\
& =\overrightarrow{0} \text {, } \\
& \begin{aligned}
\alpha\left(f_{1}, f_{2}\right)= & \frac{1}{\left|\frac{r_{1}^{2}}{r_{2}} X_{t_{1}}+\frac{r_{2}^{2}}{r_{1}} X_{t_{2}}\right|\left|r_{1} X_{t_{1}}-r_{2} X_{t_{2}}\right|}\left(\frac{r_{1}^{3}}{r_{2}} \bar{\nabla}_{X_{t_{1}}} X_{t_{1}}-\frac{r_{2}^{3}}{r_{1}} \bar{\nabla}_{X_{t_{2}}} X_{t_{2}}\right)^{\perp}= \\
= & \frac{1}{\cos ^{2} t_{3}}\left(\frac{r_{1}^{3}}{r_{2}} X_{t_{1}} t_{1}-\frac{r_{2}^{3}}{r_{1}} X_{t_{2} t_{2}}\right) \\
= & \frac{1}{\cos ^{2} t_{3}}\left(r_{1} \cos t_{3} \sin \frac{t_{1}}{r_{1}}, r_{1} \cos t_{3} \cos \frac{t_{1}}{r_{1}}\right. \\
& \left.r_{2} \cos t_{3} \sin \frac{t_{2}}{r_{2}}, r_{2} \cos t_{3} \cos \frac{t_{2}}{r_{2}}, 0\right) \\
= & \frac{1}{\cos t_{3}}\left(r_{1} \sin \frac{t_{1}}{r_{1}}, r_{1} \cos \frac{t_{1}}{r_{1}}, r_{2} \sin \frac{t_{2}}{r_{2}}, r_{2} \cos \frac{t_{2}}{r_{2}}, 0\right),
\end{aligned}
\end{aligned}
$$




$$
\begin{aligned}
\alpha\left(f_{1}, f_{3}\right) & =\frac{1}{\left|r_{1} X_{t_{1}}-r_{2} X_{t_{2}}\right|}\left(r_{1} \bar{\nabla}_{X_{t_{1}}} X_{t_{3}}-r_{2} \bar{\nabla}_{X_{t_{2}}} X_{t_{3}}\right)^{\perp}= \\
& =\frac{1}{\operatorname{css} t_{3}}\left(r_{1} X_{t_{1} t_{3}}-r_{2} X_{t_{2} t_{3}}+\left(\sin t_{3}\right) f_{1}\right) \\
& =\overrightarrow{0}, \\
\alpha\left(f_{2}, f_{3}\right) & =\frac{1}{\left|\frac{r_{1}^{2}}{r_{2}} X_{t_{1}}+\frac{r_{2}^{2}}{r_{1}} X_{t_{2}}\right|}\left(\frac{r_{1}^{2}}{r_{2}} \bar{\nabla}_{X_{t_{1}}} X_{t_{3}}+\frac{r_{2}^{2}}{r_{1}} \bar{\nabla}_{X_{t_{2}}} X_{t_{3}}\right)^{\perp}= \\
& =\frac{1}{\cos t_{3}}\left(\frac{r_{1}^{2}}{r_{2}} X_{t_{1} t_{3}}+\frac{r_{2}^{2}}{r_{1}} X_{t_{2} t_{3}}+\left(\sin t_{3}\right) f_{2}\right) \\
& =\overrightarrow{0} .
\end{aligned}
$$

isto é, $f_{3}$ gera a nulidade relativa e $f_{1}$ e $f_{3}$ geram o espaço tangente à folha que regra a hipersuperfície. Segue-se ainda, por cálculos diretos, que

$$
\left\langle\alpha\left(f_{2}, f_{2}\right), g\right\rangle=\frac{r_{1}^{2}-r_{2}^{2}}{r_{1} r_{2}} \frac{1}{\cos t_{3}}, \quad\left\langle\alpha\left(f_{1}, f_{2}\right), g\right\rangle=\frac{1}{\cos t_{3}}
$$

donde segue-se o desejado, ou seja, as curvaturas média e escalar da hipersuperfície são dadas, respectivamente, por

$$
H=\frac{1}{2} \frac{r_{1}^{2}-r_{2}^{2}}{r_{1} r_{2}} \frac{1}{\cos t_{3}}, \quad S=1-\frac{1}{3} \frac{1}{\cos ^{2} t_{3}} .
$$

Dos resultados até agora obtidos, decorre o:

Teorema 5.14 Seja $M^{n}, n \geq 3$, uma hipersuperfície conexa, regrada e de Weingarten em $\mathbb{S}^{n+1}$. Então

1. ou $M^{n}$ tem curvatura seccional constante igual a um;

2. ou $M^{n}$ é um subconjunto aberto de um fibrado normal unitário sobre uma superfície regrada mínima em $\mathbb{S}^{3}$, ou sobre um produto de dois círculos em $\mathbb{S}^{3}$.

\section{Demonstração:}

Suponha que existe $p \in M$ tal que $S(p) \neq 1$ e considere o aberto não vazio $\bar{M}=\{q \in M: S(q) \neq 1\}$. Seja $W$ uma componente conexa de $\bar{M}$.

Temos pela Observação (5.4) que a curvatura seccional de $M$ em pontos de $\bar{M}$ não é igual a 1, donde segue-se pela Observação (5.5) que $W$ é um aberto de $M$ com a propriedade que $\nu=n-2$ e portanto pelo Corolário (5.3) temos bem definido 
uma imersão $g: V^{2} \longrightarrow \mathbb{S}^{n+1}$ e uma aplicação $\psi: \Lambda^{1} \longrightarrow W \subset \mathbb{S}^{n+1}$, que é a parametrização de Gauss de $W$.

Pelo Lema (5.9), temos que o primeiro espaço normal da imersão $g, N_{1}$, em cada ponto da imagem da aplicação de Gauss tem dimensão constante igual a 1.

Por cálculos feitos na Proposição (5.10), segue-se que existe uma subvariedade totalmente geodésica $\mathbb{S}^{3} \subset \mathbb{S}^{n+1}$ tal que $g\left(V^{2}\right) \subset \mathbb{S}^{3}$ é uma superfície regrada de Weingarten que satisfaz a relação $4 H^{2}+a^{2}(K-1)=0$. Segue-se ainda pela Proposição (5.11) que $g$ determina uma imersão mínima em $\mathbb{S}^{3}$, ou $K=0$ e $H=\frac{1}{2} a$.

Se $g$ determina uma imersão mínima em $\mathbb{S}^{3}$, temos por (5.22) que $W$ verifica a primeira parte de (2), se $g$ está contida no produto de dois círculos, então segue-se por (5.26) que $W$ verifica a segunda parte de (2).

Vamos mostrar que se $\bar{M} \neq \varnothing$ então $\bar{M}=M$. Suponhamos que $\bar{M} \subset M$ não seja fechado e tomemos $q \in M$ tal que $q \in \partial \bar{M}$ e $q \notin \bar{M}$, isto é, $S(q)=1$. Seja $W$ a componente conexa de $\bar{M}$ tal que $q \in \partial W$. Então existe uma seqüência, $\left\{q_{n}\right\}_{n \in \mathrm{N}}$, de pontos pertencentes a $W$ convergindo para $q$. Mas segue-se por (5.23), (5.27) e do fato que $\sum_{j=3}^{n+1} \varphi_{j}^{2}=1$, que a curvatura escalar em $W$ é limitada por um valor diferente de 1 , o que gera uma contradição.

Logo $\bar{M}$ é fechado e portanto segue-se o desejado.

\subsection{Resultados parciais obtidos sobre a classificação das hipersuperfícies regradas e de Weingarten em $\mathbf{H}^{n+1}$}

Consideraremos, como antes, $M^{n} \subset \mathbb{H}^{n+1}$ uma hipersuperfície conexa orientada com índice de nulidade relativa constante, $\nu=n-2$, ou seja, existe uma imersão isométrica $g: V^{2} \rightarrow \mathbb{S}_{1}^{n+1}$ bem definida e $M^{n}$ pode ser parametrizada como em (5.4). Tomemos ainda, $e_{1}, e_{2}, \ldots \ldots e_{n+1}, n+1$ campos ortonormais localmente definidos em $V^{2}$, tais que $e_{1}(y)$ e $e_{2}(y)$ são tangentes a imersão $g$ em $y$ e $e_{3}(y), e_{4}(y), \ldots . e_{n+1}(y)$ geram o espaço normal a imersão $g$ em $\mathbb{S}_{1}^{n+1}$.

Proposição 5.15 Seja $M^{n} \subset \mathbb{H}^{n+1}$ uma hipersuperfície regrada conexa orientada com $\nu=n-2$. Então a imersão g é uma superfície regrada tipo espaço em $\mathbb{S}_{1}^{n+1}$.

\section{Demonstração:}


Por hipótese que podemos parametrizar $M^{n}$ localmente por

$$
\begin{aligned}
& X\left(s, t_{1}, \ldots \ldots, t_{n-1}\right)=\varphi_{0}\left(t_{1}, \ldots \ldots, t_{n-1}\right) \bar{e}_{0}(s)+\varphi_{1}\left(t_{1}, \ldots \ldots, t_{n-1}\right) \bar{e}_{1}(s)+ \\
& \quad+\sum_{i=2}^{i=n-1} \varphi_{i}\left(t_{1}, \ldots \ldots, t_{n-1}\right) \bar{e}_{i}(s),
\end{aligned}
$$

onde $\bar{e}_{j}, 2 \leq j \leq n-1$, geram a nulidade relativa e $\bar{e}_{j}, 1 \leq j \leq n-1$, geram o espaço tangente à folha que regra a hipersuperfície. Temos que a aplicação de Gauss hiperbólica, $\eta$, é paralela ao longo das folhas da nulidade relativa. que

Denotando $\eta=\frac{\bar{\eta}}{\mid \bar{\eta}}$, onde $\bar{\eta}=X_{s} \wedge X_{t_{1}} \wedge \ldots \ldots \wedge X_{t_{n-1}} \wedge X$, é fácil verificar novamente

$$
\begin{aligned}
\bar{\eta} & =X_{s} \wedge X_{t_{1}} \wedge \ldots \ldots \wedge X_{t_{n-1}} \wedge X \\
& =\sum_{j} C_{j} X_{s} \wedge \bar{e}_{0} \wedge \bar{e}_{1} \wedge \ldots \ldots \wedge \bar{e}_{n-1},
\end{aligned}
$$

onde cada $C_{j}$ depende apenas $\operatorname{dos} \varphi_{j}{ }^{6} s$, isto é, apenas dos $t_{j}{ }^{6} s$. Temos então, que fixado $s=s_{0}$, a imagem pela aplicação de Gauss hiperbólica em $\mathbb{S}_{1}^{n+1}$, da folha que regra a hipersuperfície por $s_{0}$, é ortogonal ao $n$-plano, $\Pi$, gerado por $\bar{e}_{j}, 0 \leq j \leq n-1$, portanto está contida em um 2-plano, $\mathcal{P}$.

Como $\bar{e}_{0}\left(s_{0}\right)$ é um vetor tipo tempo, temos que $\langle,\rangle_{\mid \Pi}$ é não-degenerada de índice 1 , portanto $\langle,\rangle_{\mid p}$ é positiva-definida.

Logo fixado $s=s_{0}$, a imagem da folha pela aplicação de Gauss hiperbólica, está contida em uma geodésica de tipo espaço, $\mathcal{P} \cap \mathbb{S}_{1}^{n+1}$. Como $g$ parametriza a aplicação de Gauss, temos que $g$ é a parametrização de uma superfície regrada tipo espaço em $\mathbb{S}_{1}^{n+1}$.

Segue-se pela proposição anterior, que os $n+1$ campos ortonormais, localmente definidos em $V^{2}$, podem ser tomados de forma que $e_{1}(y)$ seja tangente à folha da imersão $g$ em $y$, isto é, $\theta\left(e_{1}, e_{1}\right)=0$ onde $\theta$ é a segunda forma fundamental da imersão $g$. Com relação a esta base temos que

$$
B_{e_{j}}(y)=B_{j}=\left(\begin{array}{cc}
0 & \left\langle\theta\left(e_{1}, e_{2}\right), e_{j}\right\rangle \\
\left\langle\theta\left(e_{1}, e_{2}\right), e_{j}\right\rangle & \left\langle\theta\left(e_{2}, e_{2}\right), e_{j}\right\rangle
\end{array}\right)=\left(\begin{array}{ll}
0 & \beta_{j} \\
\beta_{j} & \lambda_{j}
\end{array}\right),
$$

onde $3 \leq j \leq(n+1)$.

Analogamente ao caso esférico, tomando-se $\left\{Y_{1}, Y_{2}, \ldots . Y_{n}\right\}$ uma base ortonormal para o espaço tangente a $M^{n}$ no ponto $(y, w)$, tal que $Y_{j}, 1 \leq j \leq n-2$ geram a nulidade relativa de $M^{n}$ em $(y, w)$, segue-se por (5.5) que a curvatura média, $H$, e a curvatura escalar, $S$, de $M^{n}$ em $(y, w)$ são dados por:

$$
H(y, w)=\frac{1}{n} \operatorname{tr} A=\frac{1}{n} \operatorname{tr} B_{w}^{-1}(y)=\frac{1}{n} \frac{\operatorname{tr} B_{w}(y)}{\operatorname{det} B_{w}(y)}
$$




$$
\begin{aligned}
S(y, w)= & \frac{1}{n(n-1)} \sum_{i, j=1, i \neq j}^{n}\left\langle R\left(Y_{i}, Y_{j}\right) Y_{i}, Y_{j}\right\rangle \\
= & -1+\frac{1}{n(n-1)} \sum_{i, j=1}^{n}\left(\left\langle\alpha\left(Y_{i}, Y_{i}\right), \alpha\left(Y_{j}, Y_{j}\right)\right\rangle+\right. \\
& \left.\quad-\left\langle\alpha\left(Y_{i}, Y_{j}\right), \alpha\left(Y_{i}, Y_{j}\right)\right\rangle\right) \\
= & -1+\frac{1}{n(n-1)} \sum_{i, j=n-1}^{n}\left(\left\langle\alpha\left(Y_{i}, Y_{i}\right), \alpha\left(Y_{j}, Y_{j}\right)\right\rangle+\right. \\
& \left.\quad-\left\langle\alpha\left(Y_{i}, Y_{j}\right), \alpha\left(Y_{i}, Y_{j}\right)\right\rangle\right) \\
= & -1+\frac{2}{n(n-1)}\left(\operatorname{det} B_{w}^{-1}(y)\right) \\
= & -1+\frac{2}{n(n-1)}\left(\frac{1}{\operatorname{det} B_{w}(y)}\right) .
\end{aligned}
$$

Observação 5.16 Analogamente ao caso esférico, sejam $M^{n} \subset \mathbb{H}^{n+1}$ uma hipersuperfície regrada de Weingarten com índice de nulidade relativa constante $\nu=n-2$ e $(y, w) \sim\left(y_{1}, y_{2}, l_{3}, l_{4}, \ldots . l_{n+1}\right)$ uma parametrização local de $M^{n}$ onde $y_{1}$ e $y_{2}$ são as coordenadas de $y$ e $w=\sum_{j=3}^{n+1} \varepsilon_{j} l_{j} e_{j}$ é um campo normal unitário à imersão $g$, onde $\varepsilon_{j}=\left\langle e_{j}, e_{j}\right\rangle$. Note que $l_{j}=l_{j}(y, w)$ tal que $\sum_{j=3, k \neq j}^{n+1} l_{j}^{2}-l_{k}^{2}=-1$ e $e_{1}, e_{2}, \ldots e_{n+1}$, $n+1$ campos ortonormais tomados como anteriormente. Usando-se a notação dada em (5.29), mostra-se que

$$
d\left(\sum_{j=3}^{n+1} l_{j} \lambda_{j}\right) \wedge d\left(\sum_{j=3}^{n+1} l_{j} \beta_{j}\right)=0 .
$$

Temos que o primeiro espaço normal da imersão $g$ em $y$ é gerado por $\theta\left(e_{1}, e_{2}\right)$ e $\theta\left(e_{2}, e_{2}\right)$, isto é, $N_{1}^{g}(y)=\operatorname{span}\left\{\theta\left(e_{1}, e_{2}\right), \theta\left(e_{2}, e_{2}\right)\right\}$. Segue-se então que $\operatorname{dim} N_{1}^{g} \leq 2$. Observe que a $\operatorname{dim} N_{1}^{g}(y) \neq 0$, para todo $y$, pois caso contrário, $g$ determinaria uma imersão totalmente geodésica em $y$, ou seja, $B_{j}=0,3 \leq j \leq n+1$. Logo, neste caso, $B_{v}$ seria singular na direção de qualquer $v=\sum_{j=3}^{n+1} \quad \varepsilon_{j} l_{j} e_{j}$ e por (5.5) teríamos que $\psi$ não determina uma imersão em $y$, o que geraria uma contradição.

Vejamos o que ocorre quando supomos que $\operatorname{dim} N_{1}^{g}=1$.

Proposição 5.17 Seja $M^{n} \subset \mathbb{H}^{n+1}$ uma hipersuperfície conexa regrada e de Weingarten com $\nu=n-2$. Suponha que a dimensão do primeiro espaço normal da imersão $g$ é constante igual a 1 . Então existe uma subvariedade totalmente geodésica $\mathbb{J}^{3} \subset \mathbb{S}_{1}^{n+1}$ tal que $g\left(L^{2}\right) \subset \mathbb{J}^{3}$ é uma superfície regrada tipo espaço e de Weingarten não desenvolvível e satisfaz $4 \mathcal{H}+a^{2}(K-1)=0$, onde a é uma constante real, $K$ é a curvatura Gaussiana da superfície e $\mathcal{H}=\langle\vec{H}, \vec{H}\rangle$, onde $\vec{H}$ é o vetor curvatura média da superfície.

\section{Demonstração:}


Usando-se a proposição (5.15), segue-se que $g$ determina uma superfície regrada tipo espaço em $\mathbb{S}_{1}^{n+1}$. Tomemos, como antes, $e_{1}, e_{2}$ campos ortonormais tangentes à imersão $g$, isto é, $N_{1}^{g}=\operatorname{span}\left\{\theta\left(e_{1}, e_{2}\right), \theta\left(e_{2}, e_{2}\right)\right\}$. Por hipótese temos que $\operatorname{dim} N_{1}^{g}=1$, suponhamos então que $e_{3}$ gera $N_{1}^{g}$.

Como o espaço normal da imersão $g$ em todo ponto é um espaço de Lorentz, seguese que o vetor $e_{3}(y)$ pode ser tipo luz, tipo tempo ou tipo espaço. Observamos, no entanto, que se $e_{3}(y)$ for tipo luz, a matriz da segunda forma fundamental da imersão $g$ em $y$ na direção $e_{3}(y)$ é uma matriz nula e portanto $\psi$, definida em (5.4), não determina uma imersão em $y$. Logo $e_{3}$ é tipo tempo ou tipo espaço, donde segue-se que $N_{1}^{g}$ é um subespaço tipo tempo ou tipo espaço, respectivamente. Observe que se $e_{3}$ for tipo tempo em um ponto, então ele permanece tipo tempo em uma vizinhança deste ponto. $\mathrm{O}$ mesmo ocorre se $e_{3}$ for tipo espaço. Logo, o tipo causal do subespaço $N_{1}^{g}$ não muda na vizinhança de um ponto.

Queremos mostrar que $N_{1}^{g}$ é paralelo, pois pelo Corolário (1.27) teremos que a imersão $g$ reduz codimensão como desejado. Analogamente à Proposição (5.10), basta mostrar que $\left(N_{1}^{g}\right)^{\perp}$ é paralelo. De fato, como $\operatorname{Tg}\left(V^{2}\right)^{\perp}=\left(N_{1}^{g}\right) \oplus\left(N_{1}^{g}\right)^{\perp}$ se $\left(N_{1}^{g}\right)^{\perp}$ é paralelo então denotando $\eta=\sum_{k=4}^{n} \varepsilon_{k} t_{k} e_{k}$ segue-se que

$$
\begin{aligned}
\left\langle\eta, e_{3}\right\rangle=0 & \Rightarrow\left\langle\eta, \nabla_{e_{j}}^{\perp} e_{3}\right\rangle=0, \quad j=1,2 \\
& \Rightarrow \nabla_{e_{j}}^{\perp} e_{3} \in N_{1}^{g} \\
& \Rightarrow N_{1}^{g} \text { é paralelo, }
\end{aligned}
$$

pois caso contrário, como $\eta$ é qualquer, $\left(N_{1}^{g}\right)^{\perp}$ seria degenerado. Mostremos então que $\left(N_{1}^{g}\right)^{\perp}$ é paralelo.

Como $\mathbb{S}_{1}^{n+1}$ é um espaço de curvatura constante, temos por (1.2) que a equação de Codazzi para a imersão $g$ é dada por

$$
\left(\nabla_{e_{1}} B\right)\left(e_{2}, \eta\right)=\left(\nabla_{e_{2}} B\right)\left(e_{1}, \eta\right)
$$

onde

$$
\left(\nabla_{e_{i}} B\right)\left(e_{j}, \eta\right)=\nabla_{e_{i}} B_{\eta} e_{j}-B_{\eta} \nabla_{e_{i}} e_{j}-B_{\nabla_{e_{i}} \eta} e_{j} .
$$

Após cálculos análogos aos feitos na Proposição (5.10), mostra-se que

$$
\left\langle\nabla_{e_{1}}^{\perp} \eta, e_{3}\right\rangle=\left\langle\nabla_{e_{2}}^{\perp} \eta, e_{3}\right\rangle=0
$$

e portanto $\left(N_{1}^{g}\right)^{\perp}$ é paralelo. Logo existe uma subvariedade totalmente geodésica $\mathbb{J}^{3} \subset \mathbb{S}_{1}^{n+1}$ tal que $g\left(L^{2}\right) \subset \mathbb{J}^{3}$, onde $\mathbb{J}^{3}$ é isométrico a $\mathbb{S}_{1}^{3}$, se $e_{3}$ for tipo tempo, ou é isométrico a $\mathbb{S}^{3}$, se $e_{3}$ for tipo espaço. 
Como $M^{n}$ é de Weingarten, segue-se por (5.32) que

$$
d(l \lambda) \wedge d(l \beta)=0
$$

Aplicando-se este produto exterior ao par $\left(e_{j}, \frac{\partial}{\partial l}\right), j=1,2$, temos que

$$
l\left(\beta d \lambda\left(e_{j}\right)-\lambda d \beta\left(e_{j}\right)\right)=0 \forall l \in \mathbb{R} .
$$

Portanto

$$
\beta d \lambda-\lambda d \beta=0
$$

donde segue-se que existe uma constante real, $a$, tal que

$$
\lambda=a \beta .
$$

Observe que $\beta \neq 0$, pois caso contrário $B_{3}$ seria uma matriz singular.

Temos então que as curvaturas Gaussiana e média da imersão $g$ verificam, respectivamente,

$$
K=1-\varepsilon \beta^{2} \quad \mathcal{H}=\frac{\varepsilon}{4} \lambda^{2},
$$

onde $\varepsilon=-1$, se $e_{3}$ for um vetor tipo tempo, ou $\varepsilon=1$, se $e_{3}$ for um vetor tipo espaço. Segue-se então de (5.33) que a imersão $g$ satisfaz a relação

$$
4 \mathcal{H}+a^{2}(K-1)=0
$$

Proposição 5.18 Seja $g: V^{2} \longrightarrow \mathbb{S}_{1}^{3} \subset \mathbb{L}^{4}$ uma superfície regrada tipo espaço não desenvolvivel em $\mathbb{S}_{1}^{3}$ tal que $4 \mathcal{H}+a^{2}(K-1)=0$. Então $\mathcal{H}=0$ ou $\mathcal{H}=\frac{-a^{2}}{4} \neq 0$ e $K=2$.

\section{Demonstração:}

Se a constante $a$ for nula, então $\mathcal{H}=0$. Caso contrário, mostraremos que $\mathcal{H}=\frac{-a^{2}}{4}$ e $K=2$.

Como $g\left(V^{2}\right) \subset \mathbb{S}_{1}^{3}$ é uma superfície regrada tipo espaço, podemos parametrizá-la localmente como em (2.13), isto é, por

$$
X(s, t)=e_{0}(s) \cos t+e_{1}(s) \sin t
$$

onde $e_{0}$ e $e_{1}$ são vetores em $\mathbb{L}^{4}$ tais que

$$
\left|e_{0}\right|=\left|e_{1}\right|=1, \quad\left\langle e_{0}, e_{1}\right\rangle=0, \quad\left\langle\dot{e}_{0}, \dot{e}_{1}\right\rangle=0, \quad\left\langle\dot{e}_{1}, \dot{e}_{1}\right\rangle=1
$$


Segue-se ainda por (2.18) que as curvaturas Gaussiana e média da superfície são dadas por

$$
K=1+\frac{Q^{2}}{P^{2}}, \quad \mathcal{H}=-\frac{\left(h-2 Q\left\langle\dot{e}_{0}, e_{1}\right\rangle\right)^{2}}{4 P^{3}} .
$$

Por hipótese, temos que a superfície satisfaz a relação $4 \mathcal{H}+a^{2}(K-1)=0$, ou seja,

$$
\frac{\left(h-2 Q\left\langle\dot{e}_{0}, e_{1}\right\rangle\right)^{2}}{P^{3}}=\frac{a^{2} Q^{2}}{P^{2}}, \quad \forall s, t
$$

donde, sem perda de generalidade, segue-se que

$$
h-2 Q\left\langle\dot{e}_{0}, e_{1}\right\rangle-a Q P^{\frac{1}{2}}=0, \quad \forall s, t .
$$

Repetindo-se o raciocínio utilizado na Proposição (5.11), mostra-se que $A(s)=$ $B(s), \forall s$, onde $A$ e $B$ estão definidos em (2.17). Portanto por (2.17) e (2.18) temos que $K=2$ e $\mathcal{H}=\frac{-a^{2}}{4}$, como queríamos demonstrar.

Proposição 5.19 Seja $M^{n} \subset \mathbb{H}_{1}^{n+1}$ uma hipersuperfície regrada conexa orientada de Weingarten com $\nu=n-2$. Se a imagem da aplicação de Gauss $g\left(V^{2}\right)$ é uma superfície mínima em $\mathbb{S}_{1}^{3}$ então $M^{n}$ é uma hipersuperfície minima em $\mathbb{H}^{n+1}$.

\section{Demonstração:}

Usando-se (5.5) temos que a demonstração é imediata. De fato, como por hipótese $g\left(V^{2}\right) \subset \mathbb{S}_{1}^{3} \subset \mathbb{S}_{1}^{n+1}$ é uma superfície regrada tipo espaço podemos escolher, como antes, $n+1$ campos ortonormais localmente definidos em $V^{2}$ com a característica adicional de que $e_{3}$ é um campo tangente a $\mathbb{S}_{1}^{3}$, isto é, em cada ponto determina um vetor tipo tempo. Nesta base, temos que a matriz da segunda forma fundamental da imersão $g$ em $y$ é dada por

$$
B_{3}=\left(\begin{array}{cc}
0 & \beta \\
\beta & \lambda
\end{array}\right), \quad B_{j}=0,4 \leq j \leq(n+1)
$$

Observe, no entanto, que $\lambda \equiv 0$, pois por hipótese segue-se ainda que $g\left(V^{2}\right)$ é uma superfície mínima.

Logo usando-se (5.5) temos que o traço da matriz da segunda forma fundamental da imersão $\psi$ é zero, e portanto $M^{n}$ é uma hipersuperfície mínima em $\mathbb{H}^{n+1}$. 
Proposição 5.20 Seja $M^{n} \subset \mathbb{H}_{1}^{n+1}$ uma hipersuperfície regrada conexa orientada de Weingarten com $\nu=n-2$. Se $g\left(V^{2}\right)$ determina uma superficie em $\mathbb{S}_{1}^{3}$ tal que $K=2$ e $\mathcal{H}=\frac{-a^{2}}{4} \neq 0$, então $M^{n}$ possui curvatura média e escalar constante em relação a dois de seus parâmetros.

\section{Demonstração:}

Analogamente à proposição anterior, podemos tomar $n+1$ campos ortonormais, tais que a matriz da segunda forma fundamental da imersão $g$ em $y$ é dada por

$$
B_{3}=\left(\begin{array}{cc}
0 & \beta \\
\beta & \lambda
\end{array}\right), \quad B_{j}=0,4 \leq j \leq(n+1) .
$$

onde $e_{3}$ é um campo tangente a $\mathbb{S}_{1}^{3}$.

Usando-se o fato que $K=2$ e $\mathcal{H}=\frac{-a^{2}}{4}$, podemos supor sem perda de generalidade, que $\beta=-1$ e $\lambda=-a$. Então segue-se por (5.4) que $M^{n}$ pode ser parametrizada localmente por

$$
X\left(t_{1}, t_{2}, s_{1}, \ldots s_{n-2}\right)=\sum_{j=3}^{n+1} \varphi_{j}\left(s_{1}, \ldots s_{n-2}\right) e_{j}\left(t_{1}, t_{2}\right)
$$

onde $w=\sum_{j=3}^{n+1} \varphi_{j} e_{j} \mathrm{e}-\varphi_{3}^{2}+\sum_{j=4}^{n+1} \varphi_{j}^{2}=-1$. Além disso, temos que a matriz da segunda forma fundamental da imersão $X$ no ponto $(y, w)$ restrito ao complemento ortogonal da nulidade relativa é dada por $B_{w}^{-1}(y)$, isto é,

$$
A_{g}=\left(\begin{array}{ll}
0 & -\varphi_{3} \\
-\varphi_{3} & -a \varphi_{3}
\end{array}\right)^{-1}
$$

Temos então por (5.30) e (5.31) que as curvaturas média e escalar da hipersuperfície são dadas, respectivamente, por

$$
H=\frac{1}{n} \frac{a}{\varphi_{3}}, \quad S=-1-\frac{2}{n(n-1)} \frac{1}{\varphi_{3}^{2}} .
$$

Observação 5.21 Ao contrário do caso esférico, não conseguimos controlar o comportamento do primeiro espaço normal da imersão $g, N_{1}^{g}=\operatorname{span}\left\{\theta\left(e_{1}, e_{2}\right), \theta\left(e_{2}, e_{2}\right)\right\}$, quando supomos que sua dimensão é constante igual a 2. Temos que o subespaço $N_{1}^{g}$ pode ser positivo definido, não degenerado de índice 1 e degenerado. Mostra-se que o primeiro caso não pode ocorrer, mas pouco se pode afirmar sobre os outros dois casos. 


\section{Referências Bibliográficas}

[BDJ] Barbosa, J.L.M., Dajczer, M., Jorge, L.P., Minimal Ruled Submanifolds in Spaces of Constant Curvature, Indiana University Mathematics Journal, vol. $33, n^{\circ} 4$ (1984).

[BD] Barbosa, J.L.M., Delgado, J.A., Ruled Submanifolds of Spaces Forms With Mean Curvature of Nonzero Constant Length, Amer. J. Math., 106 (1984) $n^{\circ} 4,763-780$.

[B] Barrientos, J.O., Superfícies helicoidais com curvatura constante no espaço de formas tridimensionais, Tese de Doutorado, PUC, Rio de Janeiro, 1995.

[Ba] Barros, M., General Helices and a Theorem of Lancret. Proc. of the Am. Math. Society 125, 1503-1509 (1997).

[Be] Beltrami, E., Risoluzione di un Problema relativo alla teoria Delle Superficie Gobbe, Ann. Mat. Pura Appl. 7, 139-150 (1865).

[BK] Blair, D.E., Koufogiorgos, Th., Ruled Surfaces with Vanishing Second Gaussian Curvature, Mh. Math. 113, 177-181 (1992).

[BL] Brito, F.G.B., Leite, M.L., A remark on rotational hypersurfaces of $S^{n}$, Bull. Soc. Math. Belg., Ser. B, 42 (1990), 303-318.

[D] Dajczer, M., Submanifolds and isometric immersions. Based on the notes prepared by Mauricio Antonucci, Gilvan Oliveira, Paulo Lima-Filho and Rui Tojeiro. Mathematics Lecture Series, 13. Publish or Perish, Inc., Houston, TX, 1990.

[DG] Dajczer, M., Gromoll, D., Gauss parametrizations and rigidity aspects of submanifolds. J. Differential Geometry 22 (1985), 1-12.

[DT] Dajczer, M., Tenenblat, K., Rigidity for complete Weingarten hypersurfaces. Trans. Amer. Math. Soc. 312 (1989), no 1, 129-140. 
[Di] Dini, U., Sulle Superficie Gobbe nelle quali Uno Dei Due Raggi di Curvatura Principale e una funzione dell' altro. Ann. Mat. Pura Appl. 7, 205-210 (1865).

[E] Erbacher, J., Reduction of Codimension of Isometric Immersions. J. Diff. Geometry. (1971), 333-340.

[F] Ferus, D., On the completeness of nullity foliations. Mich. Math. J. 18 (1971), 61-64.

[K] Kuhnel, W., Ruled W-surfaces, Arch. Math., vol 62, 475-480 (1994).

[LS1] Langer, J; Singer, D. The total squared curvature of closed curves. J. Differential Geom. 20 (1984), no. 1, 1-22.

[LS2] Langer, J; Singer, D., Knotted elastic curves in $R^{3}$. J. London Math. Soc. (2) 30 (1984), no. 3,512-520.

[L] Lawson, B., Complete minimal surfaces in $S^{3}$. Ann. of Math. 92 (1970), 335-374.

[O] O'Neill., Semi-Riemannian Geometry with Applications to Relativity. Academic Press, New York, 1983.

[P] Pinkall, U., Hopf tori in $S^{3}$ Invent. Math. 81,379-386 (1985).

[R] Ripoll, J., Superficies invariantes de curvatura média constante., Tese de Doutorado, IMPA, Rio de Janeiro, 1986.

[S] Spivak, M., Comprehensive introduction to differential geometry. vol 4.

[St] Struik, D.J., Lectures on classical Differential Geometry. Addison-Wesley Press, Inc., Cambridge, Mass., 1950. 